\title{
Problemi al contorno per i sistemi ellittici simmetrici di equazioni lineari alle derivate parziali del primo ordine a coefficienti costanti in $m(\geq 3)$ variabili indipendenti.
}

Memoria di Antonio Avantagaiati (a Napoli) (*i)

Sunto. - Come nella introdusione.

1. Introduzione. - In questo lavoro ci occuperemo principalmente del seguente

Problema I.

Assegnati :

Un dominio $T$ di $E_{m}(n \geq 3)$ di classe $\left({ }^{1}\right) A^{(2)}$;

Un sistema di eqnazioni lineari alle derivate parziali di prim'ordine

$$
\underset{s=1}{\Sigma} \sum_{p=1}^{n} a_{r s}^{p} \frac{\partial u_{s}}{\partial x_{p}}=f_{r} \quad r=1, \ldots, 2 n,
$$

simmetrico $\left(a_{r s}^{p}=a_{s r}^{p}\right)$, a coefficienti costanti reali, con $f_{r} \in C^{(0, \alpha)}(T)\left(^{2}\right)$ e verificante la condizione di ellitticità

$$
A(\lambda)=\operatorname{det}\left\|\sum_{p=1}^{m} a_{r s}^{p} \lambda_{p}\right\| \neq 0
$$

per ogni $\lambda=\left(\lambda_{1}, \ldots, \lambda_{m}\right) \in E_{m}-\{O\}$;

Un ricoprimento finito di $\partial T$, mediante gli insiemi $A_{1}, \ldots, A_{N}$, ciascuno contenuto in $\partial T$, omeomorfo ad una ipersfera (aperta) di $E_{m-1}$ ed avente contorno (frontiera $m \partial T$ ) abbastanza regolare;

Su ciascun insieme $A_{k}$ una matrice $M_{k}=\left\|c_{p q}^{(k)}\right\|$ a $n$ righe e $2 n$ colonne, ed un vettore ad $n$ componenti $c^{(k)}=\left(c_{1,0}^{(k)}, \ldots, c_{n, 0}^{(k)}\right)$ tali che ciascuna delle funzioni $c_{p q}^{(k)} p=1, \ldots, n ; q=0,1, \ldots, 2 n$ sia di classe $C^{(0, \alpha)}\left(A_{k}\right)$;

(") Lavoro eseguito nell'ambito dell'attività del gruppo di ricerche n. 13 del C.N.R. per il 1961.62 .

(1) Cfr. [20] Cap. I n. 1.

(2) Qui ed in seguito con $\alpha$ indicheremo un numero reale qualunque, maggiore di zero e minore di uno, non necessariamente sempre lo stesso nelle varie circostanze in cui interverrà. 
Determinare $2 n$ funzioni $\left\{u_{1}, \ldots, u_{2 n}\right\}$ tali che

$$
\left.u_{i} \in C^{(0, x)} \mid T\right) \cap C^{(1)}(T-\partial T) .
$$

per ogni $i=1, \ldots, 2 n$, verifichino in ogni punto di $T-\partial T$ il sistema (1.1) e su ogni $A_{k}$ le condizioni

$$
\sum_{q=1}^{2 n} c_{p q}^{(k)} u_{q}=c_{p 0}^{(k)} \quad p=1, \ldots, n
$$

ammettendo che siano verificate le seguenti ipotesi

$\left.I_{1}\right)$ In ogni punto di $A_{k}$ si ha $\left\|c_{p q}^{(k)}\right\|^{2}>0$

$I_{2}$ ) In ogni regione non vuota del tipo $A_{\imath} \cap A_{j}(i \neq j)$ è definita una coppia di matrici quadrate di ordine $n: \Gamma^{(i, i)}, \Gamma^{(i, i)}$ con

$$
\Gamma^{(r, s)}=\left\|\gamma_{h k}^{(r, s)}\right\| \in C^{(0, x)}\left(\overline{A_{\imath} \cap \bar{A}_{j}}\right),
$$

l'una inversa dell'altra e tali che in ogni punto di $A_{i} \cap A_{j}$ si abbia

$$
c_{h q}^{(i)}=\sum_{t=1}^{n} \gamma_{h t}^{(i, j)} \cdot c_{t q}^{(j)}
$$

La novità che presenta questo problema sta nel modo come sono state poste le condizioni al contorno: è assegnato un ricoprimento di $\partial T$ e su ogni aperto facente parte di esso sono assegnate $n$ combinazioni lineari indipendenti (ipotesi $I_{1}$ ) delle funzioni incognite con l'ipotesi $I_{2}$ ) che assicura l'equiva-

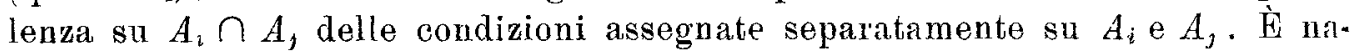
turale, però considerare insieme al precedente il

\section{Problema II.}

Assegnata una matrice $M=\left\|b_{h k}\right\|$ ed un vettore $b=\left(b_{1,0}, \ldots, b_{n, 0}\right)$ tali che ciascuna delle funzioni $b_{p q}$ sia di classe $C^{(0, \alpha)}(\partial T)$, determinare le funzioni $\left\{u_{1}, \ldots, u_{2 n}\right\}$ tali che le $u_{i} \in C^{(0, x)}(T) \cap C^{(1)}(T-\partial T)$ per $i=1, \ldots, 2 n$, verifichino in ogni punto di $T-\partial T$ il sistema (1.1) e in ogni punto di $\partial T$ le condizioni

$$
\sum_{q=1}^{2 n} b_{p q} n_{q}=b_{p 0}
$$

ammettendo che sia verificata l' ipotesi

$I_{3}$ ) In ogni punto di $\partial T$ si abbia

$$
\left\|b_{p q}\right\|^{2}>0
$$


E evidente che questo è un caso particolare del primo problema. Faremo vedere, però, con un esempio che abbiamo riportato nel n. 9), che il viceversa non è sempre vero, ciò che conferirà al problema I una notevole generalità di impostazione.

Non possiamo mancare di rilevare che esempi di condizioni al contorno di questo tipo si incontrano già nella teoria delle forme armoniche $\left(^{3}\right)$. D'altra parte sarebbe possibile porre dei problemi siffatti anche per sistemi ellittici di ordine qualunque, assegnando su ciascuno degli $A_{k}$ delle combinazioni lineari, in numero opportuno, delle funzioni incognite e delle loro derivate parziali di ordine convenientemente scelto.

Abbiamo qui affrontato lo studio del problema, traducendolo in un sistema di equazioni integrali a valor principale. Per fare ciò ci siamo serviti della matrice fondamentale dei sistemi ellittici a coefficienti costanti, che ci ha permesso di rappresentare delle soluzioni del sistema (1.1), mediante somme di potenziali di doppio strato e di dominio.

La natura delle condizioni poste su $\partial T$ ei ha suggerito di considerare su ciascuno degli $A_{k}$ un vettore $\varphi^{(k)}$ con $k_{i}=1, \ldots, N$, le componenti di $\varphi^{(i)}$ e $\varphi^{(j)}$ in ogni regione non vuota del tipo $A_{i} \cap A_{j}$ essendo legate da relazioni del tipo (1.4), e di assumere poi come momenti dei predetti potenziali di doppio strato delle diverse opportune combinazioni lineari di tali vettori. Naturalmente tali momenti vengono ad avere una diversa definizione su ciascuno degli $A_{k}$, ma le combinazioni lineari suddette sono scelte in modo che in ogni regione $A_{i} \cap A_{j}$ le due diverse definizioni di ogni momento coincidono. $L$ 'introduzione dei vettori sopra considerati richiede il preventivo studio di alcuni spazi funzionali che viene svolto nel n. 2 .

Abbiamo dedicato il n. 3 allo studio di vari teoremi della teoria del potenziale, richiamando e completando risultati che, nel caso in cui gli integrali siano estesi ad un iperpiano, sono per lo più già noti. Altri risultati sono invece da ritenersi nuovi ed hanno forse qualche interesse anche al $\mathrm{d} i$ là dei limiti di questa memoria. In questo studio ci siamo basati essenzial. mente sui teoremi di CaLderon e ZyGMund $\left({ }^{4}\right)$.

Nel n. 4 abbiamo creduto opportuno fare alcune precisazioni e sulla composizione degli integrali a valor principale estesi ad una varietà, e sulla teoria della riduzione; sopratutto abbiamo indicato come costruire l'operatore riducente, mettendo in evidenza come i procedimenti indicati da GIRaud [11] e Miflin [15] possano semplificarsi in virtù dei recenti risultati di CaLderon e Zygmund [6].

Nel n. 5, imponendo alle soluzioni del sistema (1.1), che abbiamo rappresentato mediante i potenziali di cui abbiamo parlato sopra, di verificare

(3) Cfr., per osempio, Hodge [12] e Mrranda [19].

(4) Intendiamo i teoremı $(1,1)$ e $(2,1)$ di $[5]$. 
le condizioni al contorno (1.3), abbiamo ottenuto un sistema di equazioni integrali a valor principale, $o$, più precisamente, un' equazione funzionale associata ad una trasformazione lineare $\left({ }^{5}\right)$, di uno spazio di Banach in un altro, tali spazi essendo del tipo di quelli da noi introdotti nel n. 2). Abbiamo qui in generale dato una condizione sotto la quale la trasformazione trovata e a codominio chiuso, costruendo una trasformazione riducente $\left({ }^{8}\right)$, e quindi abbiamo trovato una condizione che assicura l'esistenza di soluzioni del problema I: teorema (5.4). A questo punto ci siamo preoceupati di tradurre il problema in un' equazione equivalente.

A tale scopo, nel n. 6|, abbiamo dimostrato che l'equazione secolare associata alla forma quadratica

$$
\underset{r, s}{1, \ldots, 2 n}\left(\sum_{p=1}^{m} a_{r s}^{p} n_{p}(\xi)\right) \Lambda_{r} \Lambda_{s}
$$

ha in ogni punto $\xi \in \partial T n$ radici positive $\rho_{1}(\xi), \ldots, \rho_{n}(\xi)$ ed altrettante negative - $\rho_{1}^{\prime}(\xi), \ldots,-\rho_{n}^{\prime}(\xi)$. Ammesso (ipotesi $\left.I_{6}\right)$ ) che ciascuna radice abbia ordine di molteplicita costante al variare di $\xi$, abbiamo dimostrato che $\grave{\theta}$ possibile costruire un ricoprimento finito di $\partial T$ mediante certi insiemi che abbiamo indicato con $B_{1}, \ldots, B_{M}$, e su ogni $B_{k}(2 n)^{2}$ funzioni $d_{r, \mathrm{~s}}^{(k)}, d_{r, \mathrm{~s}}^{\prime(k)} r=1, \ldots, n$; $s=1, \ldots, 2 n$ in modo che si abbia identicamente rispetto alle $\Lambda$

$$
{ }_{r, s}^{1, \ldots}{ }^{2 n}\left(\sum_{p=1}^{m} a_{r s}^{p} n_{p}(\xi) \Lambda_{r} \Lambda_{s}\right)=\sum_{r=1}^{m}\left\{\rho_{r}\left(\sum_{s=1}^{2 n} d_{r s}^{(k)}(\xi) \Lambda_{s}\right)^{2}-\rho_{r}^{\prime}\left(\sum_{s=1}^{2 n} d_{r s}^{\prime(k)}(\xi) \Lambda_{s}\right)^{2}\right\}
$$

per ogni fissato $\xi \in B_{k}$ e ciò per $k=1, \ldots, M$; le $d_{r s}^{(k)}\left[d_{r s}^{\prime(k)}\right]$ essendo legate da condizioni analoghe alle (1.4).

Nel n. 7 abbiamo fatto vedere che, per il problema (III) che si ottiene sostituendo le $d_{r s}^{(k)}$ alle $c_{r s}^{(k)}$ nelle (1.3), vale il teorema di esistenza ed unicità, qualunque siano i termini noti, l'esistenza essendo assicurata dai risultati stabiliti al n. 5. In virtù di ciò abbiamo potuto scrivere una rappresentazione integrale di tutte le soluzioni del sistema (1.1): teorema (7.5).

Questo risultato ha permesso nel $\mathrm{n} .8 \mathrm{di}$ tradurre il problema in una equazione funzionale (sistema di equazioni integrali singolari) equivalente al problema. Abbiamo così definito l'aggiunto del problema $I$, e trovato, in virtù dei risultati relativi alla teoria delle trasformazioni riducibili in uno spazio di Banach, una condizione (sufficiente), verificata la quale, per il problema I vi sono al più un numero finito di condizione di compatibilità ed, al più, un numero finito di soluzioni linearmente indipendenti del problema omogeneo

$\left({ }^{5}\right)$ Ciò̀ omogenea additiva e continua,

(6) Cfr. [8] Cap. III n. 7. 
associato: teorema (8.4). Quindi abbiamo dimostrato un teorema di unicità per il nostro problema ed un altro per il suo aggiunto. Abbiamo cosi scritto delle condizioni per cui vi è esistenza, ed altre per cui vi è unicità.

Nel n. 9 abbiamo riportato una classe, abbastanza ampia, di sistemi ellittici simmetrici, per i quali è verificata l'ipotesi $I_{6}$ ). Ci pare notevole il fatto che, per tali sistemi, il problema che si ottiene mettendo nelle condizioni al contorno (1.3) le $d_{r s}^{(k)}$, di cui abbiamo parlato nel $\mathrm{n} .6$ ), si traduce in un sistema di equazioni integrali del tipo di Fredholm. Abbiamo poi segnalato qui un caso in cui il problema II ammette infinite condizioni di compatibilità. Infine abbiamo mostrato come si presentino naturali condizioni al contorno del tipo (1.3) e cireostanze in cni queste non possono ridursi a quelle del tipo (1.5).

2. Notazioni e generalità. - Siano: $E_{k}$ lo spazio reale euclideo a $k$ dimensioni, $y=\left(y_{1}, \ldots, y_{k}\right)$ ed $x=\left(x_{1}, \ldots, x_{k}\right)$ dae punti qualunque di $E_{k}, y-x$ il vettore avente per componenti $y_{1}-x_{1}, \ldots, y_{k}-x_{k} ; O$ il vettore nullo (origine di $E_{k}$ ), $|y-\dot{x}|$ il modulo di $y-x, y \cdot x$ il prodotto scalare di $y$ per $x ; \Omega_{k}(x, r)$ l'insieme di tutti i punti $x^{\prime}$ di $E_{k}$ tali che $\left|x^{\prime}-x\right|<r$ per ogni punto $x$ e qualungue sia il numero positivo $r$, [per brevità porremo $\Omega_{k}$ al posto di $\left.\Omega_{k}(0,1)\right]$.

Assegnato un dominio $T$ di classe $A^{(2)}$ in un fissato $E_{m}(m \geq 3)$, siano: $\partial T$ la sua frontiera, $\xi$ un punto qualunque di $\partial T, \Pi_{\xi}$ ed $n_{\xi}$ lः iperpiano tangente e la normale interna a $\partial T$ in $\xi, n(\xi)$ e versore di $n \xi,\left[\xi, \tau_{1}, \ldots, \tau_{m}\right]$ un sistema di coordinate ortogonali con l'origine in $\xi$ congruente a quello fisso di riferimento ed avente $\tau_{m}$ coincidente con $n \xi$. Se $B$ è un insieme di punti contenuto in $\partial T$ indicheremo con $f B$ la frontiera di $B$ su $3 T$.

Indicheremo con $\{A\}_{N}$ un ricoprimento finito di $\partial T$ mediante $N$ insiemi $A_{1}, \ldots, A_{N}$ ciascuno contenuto in $\partial T$ ed omeomorfo ad $\Omega_{m-1}$, tali che $A_{i} \cap A_{j}$, se non è vuoto è connesso. Supporremo sempre di aver associato ad ogni punto $\xi \in A_{k}$ un sistema di coordinate che vari con continuità (in senso orvio) al variare di $\xi$ in $A_{k}$, e ciò per ogni $k=1, \ldots, N$. Tale sistema di coordinate sarà indicato col simbolo $\left[\xi, \tau_{1}, \ldots, \tau_{n l}\right]_{k}$. Dato un ricoprimento $\{A\}_{N}$, non è restrittivo ammettere che sia verificata la seguente condizione:

Esiste un numero reale positivo $d$ tale che, comunque si consideri un punto $\xi \in \partial T$, vi sia un intorno di $\xi$ su $\partial T, I(\xi)$, ed un elemento $A_{h}$ di $\{A\}_{N}$ contenente $I(\xi)$, tali che $I(\xi)$ abbia per proiezione sull' iperpiano $\Pi_{\xi}$ l'ipersfera $\Omega_{m}(\xi, d) \cap \Pi_{\xi}$ e rispetto al sistema di coordinate $\left[\xi, \tau_{1}, \ldots, \tau_{m}\right]_{h}$ sia suscettibile di una rappresentazione del tipo

$$
\tau_{m}=f_{h}(\xi, t) \quad t \in \Omega_{m,-1}(O, d),
$$

avendo posto, come faremo sempre in seguito, $t=\left(\tau_{1}, \ldots, \tau_{m-1}\right)$; la funzione $f_{h}(\xi, t)$ risultando, per ogni fissato $\xi$, di classe $C^{(2)}$ in $\Omega_{m-1}(O, d)$; esiste 
inoltre una costante $\left.c\right|^{7}$ ) indipendente da $\xi$ e $h$ in modo che si abbia

$$
\left|\frac{\partial f_{h}(\xi, t)}{\partial \tau_{i}}\right| \leq c|t| \quad|t|=\left(\tau_{1}{ }^{2}+\ldots+\tau_{m-1}^{2}\right)^{1}
$$

per ogni $i=1, \ldots, m-1$.

Per ogni numero positivo $\varepsilon<d$ indicheremo con $I(\xi, \varepsilon)$ la parte di $I(\xi)$ la cui proiezione sn $\Pi_{\xi}$ coincida con $\Omega_{m}(\xi, \varepsilon) \cap \Pi_{\xi}$.

Se $F$ è una funzione definita in $E_{m}$, con $F_{k}\left(\xi, t, \tau_{m}\right)$ indicheremo il valore che essa assume in $y-\xi$, se le componenti di $y-\xi$ rispetto al sistema $\left[\xi, \tau_{1}, \ldots, \tau_{m}\right]_{k}$ sono $\left(\tau_{1}, \ldots, \tau_{m-1}, \tau_{m}\right)$. Inoltre, se $\varphi$ è una funzione definita su $\partial T$, con $\varphi_{k s}(t)$ indicheremo il valore che la $\varphi$ assume in un punto $y \in A_{k}$, se le componenti di $y-\xi$ rispetto al sistema $\left[\xi, \tau_{1}, \ldots, \tau_{m}\right]_{k}$ sono $\left[\tau_{1}, \ldots, \tau_{m-1}, f_{k}(\xi, t)\right]$. Ometteremo l'indice $k$ quando non possono sorgere equivoci.

Introduciamo ora alcuni spazi di Banach di eni dovremo servirci in seguito. Dato un ricoprimento $\{A\}_{N}$ di $\partial T$ supponiamo che su ciascun insieme non vuoto del tipo $A_{i} \cap A_{j}(i \neq j)$ sia definita una coppia di matrici quadrate di ordine $n:\left[\Gamma^{(i, j)}, \Gamma^{(j, i)}\right]$ che siano l' una l'inversa dell'altra, ciascuna avente il determinante diverso da zero su $\overline{A_{i} \cap \bar{A}_{1}}$, e tali che in ogni punto di ciascun insieme non vaoto del tipo $A_{k} \cap A_{l} \cap A_{h}$ si abbia

$$
\Gamma^{(h, k)}=\Gamma^{(h, l)} \cdot \Gamma^{(l, k)}
$$

ossia

$$
\gamma_{r s}^{(h, k)}=\sum_{t=1}^{n} \gamma_{r s}^{(h, l)} \gamma_{t s}^{(l, k)}
$$

Un insieme di matrici siffatte sarà indicato col simbolo $\{\Gamma\}_{A, n}$ o con $\{\Gamma\}_{A}$. Fissato un ricoprimento $\{A\}_{N}$ ed un insieme di matrici $\{\Gamma\}_{A}$, tale che gli elementi di ciascuna matrice $\Gamma^{(i, j)}$ siano funzioni di classe $C^{(0, \alpha)}$ jn $A_{\imath} \cap A_{\jmath}$, indicheremo con $C^{(0, \alpha)}\left(\{\Gamma\}_{A}\right)$ l'insieme delle $N$-ple di vettori $\left(\left(b^{(1)}, \ldots, b^{(N)}\right)\right)$ ciascuno dei quali sia ad $n$ componenti reali $b^{(i)}=\left(b_{1}^{(i)}, \ldots, b_{n}^{(i)}\right)$ con $b_{h}^{(l)} \in C^{(0, \alpha)}\left(A_{\imath}\right)$ per ogni $h=1, \ldots, n$ per ogni $i=1, \ldots, N$, e tali che in ogni punto di $A_{i} \cap A_{j}$ verifichino la condizione

$$
b^{(i)}=\Gamma^{(i, j)} \cdot b^{(j)}
$$

(7) Con le lettere $c$ e $\beta$ indicheremo aubitrarie costanti reali positive, non necssariamente sempre le stesse nei vari casi in cui interverranno. 
ossia

$$
b_{h}^{(i)}=\sum_{k=1}^{n} \gamma_{h i c}^{(i, j)} b_{k}^{\langle j\rangle} .
$$

Si comprende che le condizioni (2.3) cui soddisfano le matrici $\Gamma^{(i, j)}$ servono per dare coerenza alle (2.4) nei punti di $\partial T$ che appartengono a più di due olementi di $\{A\}_{N}$.

Detti $b^{\prime}=\left(\left(b^{\prime(1)}, \ldots, b^{\prime(N)}\right)\right)$ e $b^{\prime \prime}=\left(\left(b^{\prime \prime(1)}, \ldots, b^{\prime \prime(N)}\right)\right)$ due elementi qualunque di $C^{(0, \alpha)}\left(\{\Gamma\}_{A}\right)$ ed $r^{\prime}$ e $r^{\prime \prime}$ due qualunque numeri reali, poniamo

$$
r^{\prime} b^{\prime}+r^{\prime \prime} b^{\prime \prime}=\left(\left(r^{\prime} b^{\prime(1)}+r^{\prime \prime} b^{\prime \prime(1)}, \ldots, r^{\prime} b^{\prime(N)}+r^{\prime \prime} b^{\prime \prime(N)}\right)\right) ;
$$

in tal modo $C^{(0, \alpha)}\left\{\{\Gamma\}_{A}\right\}$ diventa uno spario lineare nel corpo dei numeri reali, essendo evidente che la $N$-pla di vettori a secondo membro della (2.5) soddisfa ancora alle (2.4).

Introduciamo in $C^{(0, \alpha)}\left(\{\Gamma\}_{A}\right)$ la seguente norma

$$
\|b\|_{C^{(0, \alpha)}}=\sum_{k=1}^{N} \sum_{h=1}^{n}\left\{\sup _{\xi \in A_{k}}\left|b_{h}^{(k)}(\xi)\right|+\left[b_{h}^{(k)}\right]_{\alpha}\right\}
$$

ove si ̀̀ indicato con $[\ldots]_{\alpha}$ il coefficiente di HöLDER di esponente $\alpha$ della funzione racchiusa in parentesi quadre.

Si coustata facilmente che, rispetto a tale norma, $C^{(0, \alpha)}\left(\{\Gamma\}_{A}\right) \dot{\theta}$ uno spazio completo.

Analogamente si definisce lo spazio $C^{(0)}\left(\left\{\Gamma_{A}\right)\right.$ in cui la norma è introdotta ponendo

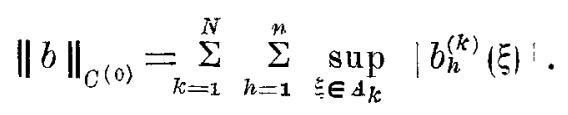

Infine, fissato come sopra un ricoprimento $\{A\}_{N}$ di $\partial T$ ed un insieme di matrici $\{\boldsymbol{\Gamma}\}_{A}$, tale che gli elementi di ciascuna matrice $\Gamma^{(i, j)}$ siano fun. zioni continue e limitate in $A_{2} \cap A_{1}$, indicheremo con $L^{(2)}\left(\{\Gamma\}_{A}\right)$ l'insieme delle $N$-ple di vettori $\left(\left(b^{(1)}, \ldots, b^{(N)} \|\right)\right.$ ciascuno dei quali sia ad $n$ componenti $b^{(i)}=\left(b_{1}^{(i)}, \ldots, b_{n}^{(i)}\right)$ con $b_{h}^{(i)} \in L^{(2)}\left(A_{i}\right)$ per ogni $h=1, \ldots, n$ ed $i=1, \ldots, N$ e tali che in quasi tutti i punti $A_{\imath} \cap A_{j}$ verifichino la condizione (2.4).

Se $b \in L^{(2)}\left(\{\Gamma\}_{A}\right)$ definiremo la norma di $b$ ponendo

$$
\|b\|_{L^{(2)}}=\sum_{i=1}^{N} \sum_{h=1}^{n}\left\{\int_{A_{i}}\left|b_{h}^{(i)}(\xi)\right|^{2} d \xi \sigma\right\}^{\frac{1}{2}}
$$


Si riconosee cbe, con tale definizione di norma $L^{(2)}\left(\{\Gamma\}_{A}\right\}$ ì completo.

Fissato un sistema di matrici $\{\Gamma\}_{A}$. poniamo:

$$
\gamma_{r i}^{*(i, j)}=\gamma_{3 *}^{(j, i)}
$$

e quindi

$$
\Gamma^{*(i, j)}=\left\|\gamma_{r s}^{*(i, j)}\right\|
$$

Indicheremo con $\left\{\mathrm{I}^{* *}\right\}_{A}$ l'insieme delle coppie di matrici cosi definite. Si dimostrano facilmente $\mathrm{i}$ seguenti

Lemma $(2.1)-S e \quad b=\left(\left(b^{(1)}, \ldots, b^{(N)}\right)\right) \in C^{(0, \alpha)}\left(\{\Gamma\}_{A}\right) \quad e \quad c=\|\left(c^{(1)}, \ldots\right.$, $\left.\left.c^{(N)}\right)\right\} \in C^{\langle 0, \alpha)}\left(\left\{\Gamma^{*} \xi_{A}\right)\right.$, per ogni punto $\xi \in A_{\boldsymbol{l}} \cap A_{k}$ si ha

$$
\sum_{i=1}^{n} b_{i}^{(h)}(\xi) c_{i}^{(h)}(\xi)=\sum_{i=1}^{n} b_{i}^{(k)}(\xi) c_{i}^{(k)}(\xi)
$$

qualunque siano $h$ e $k$ tali che $A_{h} \cap A_{k}$ sia non vuoto.

Lemma (2.2) - Comunque si prenda un elemento $b \in C^{(0, \alpha)}\left(\{\Gamma\}_{A}\right)$ ed uno $c \in C^{(0, \alpha)}\left(\left\{\Gamma^{*}\right\}_{A}\right)$, ponendo per ogni punto $\xi \in \partial T$

$$
\varphi(\xi)=\sum_{i=1}^{n} b_{i}^{(k)}(\xi) c_{i}^{(k)}(\xi)
$$

se $\xi \in A_{k}$, si definisce una funzione di classe $C^{(0, \alpha)}(\partial T)$.

Il primo si riduce ad una semplice verifica; la tesi del secondo è immediata conseguenza di quella del primo, in quanto questa assicura l'univocita della definizione della $\varphi$ in ogni punto $\xi$ di $\partial T$ e permette de dedurre la hölderianità globale della $\varphi$ da quella locale.

Fissato un ricoprimento $\{A\}_{N}$, indicheremo con $\mathbb{D}_{A}$ una decomposizione di $\partial T$ mediante $N$ insiemi $D_{1}, \ldots, D_{N}$ chiusi su $\partial T$ ciascuno omeomorfo ad una ipersfera e tali che $D_{k} \subset A_{k}$ per $k=1, \ldots, N$; quindi indicheremo con $L_{n}^{(2)}\left(D_{k}\right)$ l'insieme dei vettori ad $n$ componenti reali ciascuna di quadrato sommabile su $D_{k}$, e con $H_{n}\left(\Phi_{A}\right)$ o $H\left(\Phi_{A}\right)$ il prodotto combinatorio

$$
L_{n}^{(2)}\left(D_{1}\right) \times \ldots \times L_{n}^{(2)}\left(D_{N}\right) .
$$

Posto, analogamente a quanto abbiamo fatto sopra, $f^{(i)}=\left(f_{1}^{(i)}, \ldots, f_{n}^{(i)}\right)$ con $f_{h}^{(i)} \in L^{(2)}\left(D_{\imath}\right)$, un elemento generico di $H_{n}\left(\Phi_{A}\right)$ lo indicheremo col simbolo $f=\left(\left(f^{(1)}, \ldots, f^{\left(N^{\prime}\right)}\right)\right)$; dati due elementi $f$ e $g$ di $H_{n}\left(\mathbb{D}_{A}\right)$, definiremo il loro 
prodotto scalare al modo consueto, ponendo cioè

$$
(f, g)=\sum_{i=1}^{N} \int_{D_{i}}\left(\sum_{h=1}^{n} f_{h}^{(i)} g_{h}^{(i)}\right) d \sigma .
$$

Teorema (2.1) - Dato un sistema di coppie di matrici $\{\mathrm{I}\}_{A}$ ed una qualunque decomposizione $\Phi_{A}$ di $\partial T$, gli spazi $L^{(2)}\left(\{\Gamma\}_{A}\right)$ e $H\left(\Phi_{A}\right)$ sono isomorfi, esiste cioè una trasformazione lineare biunivoca dell' uno sull'altro.

Infatti, detto $b=\left(\left(b^{(1)}, \ldots, b^{(N)}\right)\right)$ un elemento di $L^{(2)}\left(\{\Gamma\}_{A}\right)$, consideriamo la $N$-pla di vettori $f^{(1)}, \ldots, f^{(N)}$ così definiti :

$$
f^{(l)}=\text { restrizione a } D_{l} \text { di } b^{(l)}
$$

è evidente che $\left(\left(f^{(1)}, \ldots, f^{(N)}\right)\right)$ è un elemento di $H\left(\Psi_{A}\right)$ e che la trasforma. zione definita dalla (2.11), diciamola $\tau_{D}$, è omogenea e distributiva.

Per dimostrare che si tratta di una trasformazione su $H\left(\mathbb{\Psi}_{A}\right)$, fissiamo un qualunque elemento $\left(\left(f^{(1)}, \ldots, f^{(N)}\right)\right)$ di tale spazio.

Consideriamo quindi per ogni fissato $k$ il vettore $b^{(k)}$ definito dalle seguenti posizioni

$$
\begin{array}{rlrl}
b^{(k)}(\xi) & =f^{(k)}(\xi) & & \text { se } \xi \in D_{k}-f D_{k} \\
》 & =\Gamma^{(k, h)}(\xi) \cdot f^{(h)}(\xi) & \text { se } \xi \in\left(D_{h}-f D_{h}\right) \cap A_{k} \neq \emptyset .
\end{array}
$$

In tal modo $b^{(k)}$ è definito quasi ovunque su $A_{k}$. Fissiamo ora un punto $\xi \in A_{i} \cap A_{j}$; allora se $\xi \in D_{i}-f D_{i}$ si ha :

$$
b^{(j)}(\xi)=\Gamma^{(j, i)}(\xi) \cdot f^{(i)}(\xi)=\Gamma^{(j, i)}(\xi) \cdot b^{(i)}(\xi)
$$

per il modo come sono stati definiti $b^{(j)}$ e $b^{(i)}$; se

$$
\xi \in\left(D_{l}-f D_{l}\right) \cap A_{i} \cap A_{\jmath}
$$

si ha in virtù della (2.3) :

$$
\begin{aligned}
b^{(j)}(\xi) & =\Gamma^{(j, l)(\xi)} \cdot f^{(l)} \\
& =\Gamma^{(j, i)} \cdot \Gamma^{(i, l)} \cdot f^{(l)}=\Gamma^{(j, i)} \cdot b^{(i)} .
\end{aligned}
$$

Ciò prova che $b=\left(\left(b^{(1)}, \ldots, b^{(N)}\right)\right)$ è un elemento di $L^{(2)}\left(\{\mathbf{\Gamma}\}_{A}\right)$. Poichè risulta ovviamente

$$
f=\tau_{D}(b)
$$


si ha che $\tau_{D}$ è una trasformazione di $L^{(2)}\left(\{\boldsymbol{\Gamma}\}_{A}\right)$ su $H_{n}\left(\Phi_{A}\right)$; che essa sia biunivoca e lineare è poi inmediato.

Tale risultato si estende allo spazio $L^{(2)}\left(\left\{\Gamma^{*}\right\}_{2}\right)$, riguardo al quale la trasformarione analoga a quella che abbiamo indicato con $\tau_{D}$ sarà indicata $\operatorname{con} \mathcal{T}_{D}^{*}$.

Teorema (2.2) - Comunque si consideri un funzionale lineare $F$ in $L^{(2)}\left(\{\Gamma\}_{A}\right)$, esiste un elemento $b \in\left(L^{(2)}\left(\left\{\Gamma^{(i)}\right\}_{A}\right)\right.$, tale che per ogni $\varphi \in L^{(5)}\left(\{\Gamma\}_{A}\right)$ si $a b b i a$

$$
F(\varphi)=\sum_{i=1}^{N} \int_{D i} \varphi^{(i)} \cdot b^{(i)} d \sigma=\left(\tau_{D}(\varphi), \tau_{D}^{*}(b)\right)
$$

qualunque sia la decomposizione $\mathbb{\Phi}_{A}$ di $\partial T$, ed avendo indicato con $($,$) il$ prodotto scalare in $H\left(\Phi_{A}\right)$.

Infatti, considerata una qualunque decomposizione $\mathbb{D}_{A}^{\prime}$ di $\partial T$. poniamo per ogni $\varphi \in L^{(2)}\left(\{\Gamma\}_{A}\right)$

$$
\begin{aligned}
\varphi^{\prime} & =\tau_{D^{\prime}(\varphi)} \\
F(\varphi) & =F\left(\tau_{D}^{-1}\left(\varphi^{\prime}\right)\right)=F^{\prime}\left(\varphi^{\prime}\right) ;
\end{aligned}
$$

in tal modo si viene a definire in $H\left(\mathbb{D}_{A}^{\prime}\right)$ nn funzionale $F^{\prime}$ che si dimostra facilmente essere lineare. Dalla forma del più generale funzionale lineare in $H\left(\boldsymbol{D}_{A}^{\prime}\right)$, si deduce che esiste un elemento $f^{\prime} \in H\left(\mathbb{\Phi}_{A}^{\prime}\right)$ tale che si abbia $\left.{ }^{8}\right)$

ossia

$$
F(\varphi)=F^{\prime}\left(\varphi^{\prime}\right)=\left(f^{\prime}, \quad \widetilde{\tau}_{D^{\prime}}(\varphi)\right)
$$

$$
F(\varphi)=\sum_{i=1}^{N} \int_{D^{\prime} i} \varphi^{(i)} \cdot f^{\prime(i)} d \sigma
$$

qualunque sia $\varphi$.

Se ora si considera un'altra decomposizione $\mathbb{P}_{A}^{\prime \prime}$ di $\partial T\left\{D_{1}^{\prime \prime}, \ldots, D_{N}^{\prime \prime}\right\}$, e si suppone

$$
D_{l}^{\prime}=D_{l}^{\prime}
$$

per ogni $l$ diverso da $h$ e da $k(h \neq k)$, e

$$
D_{k}^{\prime} \cup B=D_{k}^{\prime \prime} ; \quad D_{h}^{\prime}=D_{h}^{\prime \prime} \cup B \quad \text { con } B \subset A_{h} \cap A_{k}
$$

(8) Cfr., per esempio, [2]. 
Si troverà un elemento $f^{\prime \prime} \in H\left(\Phi_{A}^{\prime \prime}\right)$ tale che risulti

$$
\left(f^{\prime \prime}, \tau_{D^{\prime \prime}}(\varphi)\right)=\left(f^{\prime}, \tau_{D^{\prime}}(\varphi)\right) .
$$

Sfruitando l'arbitrarietà di $\varphi$ si deduce che su $B$ deve arersi:

$$
\int_{B} \varphi^{(k)} \cdot f^{\prime \prime}(k) d \sigma=\int_{B} \varphi^{(h)} \cdot f^{\prime(h)}=\int_{B}\left(\Gamma^{(h, k)} \cdot \varphi^{(k)}\right) \cdot f^{\prime(h)} d \sigma
$$

e quindi quasi ovunque

$$
f^{\prime \prime(k)}=f^{\prime(h)} \cdot \mathrm{I}^{(h, k)}
$$

Da questa si ricava facilmente

$$
\tau_{D^{\prime}}^{*-1}\left(f^{\prime}\right)=\tau_{D^{\prime \prime}}^{*-1}\left(f^{\prime \prime}\right)
$$

il nostro asserto segue allora dalla arbitrarietà con cui si può scegliere $B$ in $A_{h} \cap A_{k}$ e ponendo $b=\tau_{D^{\prime}}^{*-1}\left(f^{\prime}\right)$.

Fissati due ricoprimenti. $\{A\}_{N}$ e $\{B\}_{M}$ e due sistemi di coppie di matrici \{\}$_{A}$ e $\{\Theta\}_{B}$, sia definita su ogni insieme del tipo $A_{p} \times B_{q}$ una matrice quadrata $\mathscr{T K}^{(p, q)}$ di ordine $n$; per ogni $\xi \in A_{p}$ ed $\eta \in B_{q}$ porremo

$$
\mathfrak{9 K}^{(p, q)}(\xi, \eta)=\left\|K_{i_{j}}^{(p, q)}(\xi, \eta)\right\| \text {. }
$$

Queste $N M$ matrici, ottenute facendo variare $p$ da 1 ad $N$ e $q$ da 1 ad $M$, verifichino le seguenti condizioni:

1) fissati $\xi$, $i$, $p$, la $M-p l a$ di vettori ad $n$ componenti

$$
\left(K_{i 1}^{(p, q)}(\xi, \eta), \ldots, K_{i n}^{(p, q)}(\xi, \eta)\right)
$$

ottenuta facendo variare $q$ da 1 ad $M$, appartiene come funzione di $\eta$ allo spazio $C^{(0)}\left(\{\Theta\}_{B}\right)$ e ció comunque si fissi $\xi$ in $A_{p}$, per ogni $i$ e per ogni $p=1, \ldots, N$.

2) Fissati $\eta, j, q$ la $N-p l a$ di vettori

$$
\left(K_{1 j}^{(p, q)}(\xi, \eta), \ldots, K_{i j}^{(p, q)}(\xi, \eta)\right)
$$

ottenuta facendo variare $p$ da 1 ad $N$, come funzione di $\xi$ appartiene allo spazio $C^{(0)}\left(\{\Gamma\}_{A}\right)$.

Per esprimere che le $N M$ matrici verificano le condizioni 1) e 2), diremo che costituiscono un sistema di matrici di classe $C^{(0)}\left(\{\Gamma\}_{A},\{\Theta\}_{B}\right)$. Analoga- 
mente diremo che costituiscono un sistema di matrici di classe $N^{(\beta)}\left\{\{\mathbf{\Gamma}\}_{A}\right.$, $\left.\{\Theta\}_{B}\right\}$ quando verificheranno le ipotesi 1) e 2) vincolate alla ulteriore condizione $\xi \neq \eta$ e si ha inoltre

$$
K_{i j}^{(p, q)}(\xi, \eta)=0\left(|\eta-\xi|^{\beta-m+1}\right)
$$

qualunque viano $i, j, p$ e $q$.

Fissiamo ora un sistema di matrici $\left\|K_{i j}^{(p, q)}(\xi, \eta)\right\|$ che sia un elemento di $N^{(\beta)}\left(\left\{\boldsymbol{\Gamma}_{A}\right),\{\Theta\}_{B}\right)$ con $\beta>0$; quindi osserviamo che per ogni $\varphi \in L^{(2)}\left(\left\{\Theta^{*}\right\}_{B}\right)$, ponendo

$$
\widehat{\varphi_{i}}\left\langle(p)(\xi)=\sum_{q=1}^{M} \int_{D^{\prime} q} \sum_{j=1}^{n} K_{i, j}^{(p, q\rangle}(\xi, \eta) \varphi_{j}^{\langle q\rangle}(\eta) d_{\eta} \sigma\right.
$$

si definisce una trasformazione continua nello spazio $L^{(2)}\left(\left\{\Theta^{*}\right\}_{B}\right)$, avente codominio nello spazio $L^{(2)}\left(\{\Gamma\}_{A}\right)$ (essendo $D_{1}^{\prime}, \ldots, D_{M}^{\prime}$ una decomposizione $\Phi_{B}$ qualunque di $\partial T$, tale che $D_{q}^{\prime} \subset B_{q}$ ). In virtù del Lemma (2.1) si riconosce che tale trasformazione non dipende da $\mathbb{D}_{B}^{\prime}$.

Siano $\mathfrak{B}_{1}$ e $\mathfrak{H}_{2}$ due spazi di BANACH, $\mathfrak{S}$ ed $\mathfrak{S}^{\prime}$ due trasformazioni lineari, la prima di $\mathbb{B}_{1}$ in $\mathfrak{B}_{2}$, la seconda di $\mathfrak{J B}_{2}$ in $\mathfrak{J}_{1}$. Si dirà che $\mathfrak{S}^{\prime}$ è riducente S se

$$
\mathfrak{S}^{\prime} \mathfrak{S}=\mathfrak{d}+\tau,
$$

essendo $\mathfrak{J}$ l'identità e $\mathcal{C}$ una trasformazione lineare di $\mathfrak{H}_{1}$ in $\mathfrak{G}_{1}$, completamente continua; in tal caso si dirà anche che $\mathcal{S}$ è riducibile.

Sussiste il $\left({ }^{9}\right)$.

Teorema (2.5) - Ogni trasformazione riducibile è a codominio chiuso.

3. Premesse di teoria del potenziale. - In questo numero stabiliremo alcune proprietà per la funzione

$$
w(x)=\int_{\partial T} \frac{\Phi(\eta-x)}{|\eta-x|^{m-1}} \varphi(\eta) d_{\eta} \sigma
$$

detta potenziale generalizzato di doppio strato. Consideriamo le seguenti ipotesi

(9) Nel caso in cui $\mathfrak{H}_{1}$ coincide con $\mathfrak{H G}_{2}$ questo teorema è stato dimostrato da FrCHERA in [8], l'estenzione al caso generale si deve ad S. G. Mihlin [17]. Precedentemente lo stesso Mihlin aveva considerato il problema nel caso in cui $\mathfrak{J B}_{1}$ supposto coincidente con $\mathfrak{J B}_{2}$ è uno spazio di Hilbert. È bene avvertire che Mihlin chiama regolarizzabili e regolarizzatori gli operatori (trasformazioni) che noi, seguendo FICHERA, abbiamo denominato riducibili e ridu. centi rispettivamente. 
$\left.\mathrm{I}_{4}\right) \Phi$ è positivamente omogenea di grado zero in $E_{n}-\{O\}$ e verifica per ogni $x \neq 0$ la condizione

$$
\int_{\{|y|=1\} \cap\{x \cdot y=0\}} \Phi(y) d_{y} \sigma=0 .
$$

$\left.\mathrm{I}_{5}\right)$ Comunque si prendano due punti $\boldsymbol{y}^{\prime}$ e $\boldsymbol{y}^{\prime \prime}$ appartenenti a $\partial \boldsymbol{\Omega}_{m}$ si ha

$$
\left|\Phi\left(y^{\prime}\right)-\Phi\left(y^{\prime \prime}\right)\right| \leq c\left|y^{\prime}-y^{\prime \prime}\right|
$$

essendo $c$ una costante positiva indipendente da $y^{\prime}$ e $y^{\prime \prime}$ su $\partial \boldsymbol{Q}_{m}$.

Vogliamo rilevare esplicitamente che ogni funzione dispari verifica certamente la condizione (3.1).

Teorema (3.1) - Nelle ipotesi $\left.\mathrm{I}_{4}\right)$ e $\left.\mathrm{I}_{5}\right)$, se $\varphi \in C^{(0, \alpha)}(\partial T)$, in ogni punto $\xi \in \supset T$ si ha:

$$
\begin{aligned}
& \lim _{\xi \rightarrow 0} \int_{\partial T-I(\xi, \varepsilon)} \frac{\Phi(\eta-\xi \mid}{|\eta-\xi|^{m-1}} \varphi(\eta) d_{\eta} \sigma=\int_{\partial T-I(\xi)} \frac{\Phi(\eta-\xi)}{|\eta-\xi|^{m-1}} \varphi(\eta) d_{r_{i}} \sigma \\
& \left.+\int_{I(\xi)} \frac{\Phi(\eta-\xi)}{|\eta-\xi|^{m-1}} \mid \varphi(\eta)-\varphi(\xi)\right] d_{\eta \eta} \sigma+\varphi(\xi) \int_{|| t|<d|} H_{k}(\xi, t) d t
\end{aligned}
$$

essendo $I(\xi) \subset A_{k}$ ed avendo posto

$$
\begin{aligned}
& H_{k}(\xi, t)=\frac{\Phi_{k}\left(\xi, t, f_{k}(\xi, t) \mid\right.}{\left[|t|^{2}+f_{k}^{2}(\xi, t)\right]^{\frac{m-1}{2}}} J_{k}(\xi, t)-\frac{\Phi_{k}(\xi, t, 0)}{|t|^{m-1}} \\
& J_{k}(\xi, t)=\left\{1+\sum_{i=1}^{m-1}\left[\frac{\partial}{\partial \tau_{i}} f_{k}(\xi, t)\right]^{2}\right\}^{\frac{1}{2}}
\end{aligned}
$$

La dimostrazione è basata sulla scomposizione seguente

$$
\begin{array}{r}
\int_{\partial T-I(\xi, s)} \frac{\Phi(\eta-\xi)}{|\eta-\xi|^{m-1}} \varphi(\eta) d_{\eta} \sigma=\int_{\partial T-I(\xi)} \frac{\Phi(\eta-\xi)}{|\eta-\xi|^{m-1}} \varphi(\eta) d_{\eta_{1} \sigma} \\
+\int_{I(\xi), \varepsilon)} \frac{\Phi(\eta-\xi)}{|\eta-\xi| m-1}[\varphi(\eta)-\varphi(\xi)] d_{\eta_{1} \sigma}+\varphi(\xi) \int_{\{\varepsilon<|t|<d\}} H_{k}(\xi, t) d t,
\end{array}
$$

sul fatto che l'ipotesi $\mathrm{I}_{4}$ ) assicura che

$$
\int_{\{|t|=1\}} \Phi_{k}(\xi, t, 0) d_{t} \sigma=0
$$


e sulla maggiorazione

$$
\left|H_{k}(\xi, t)\right| \leq c \quad|t|^{2-m}
$$

che si stabilisce facilmente sfruttando l'ipotesi $I_{5}$ ) e la (2.2).

Ci proponiamo ora di ricavare una espressione del secondo membro della (3.2) in un punto $\bar{\xi}$ mediante un integrale a valor principale relativo ad una regione piana. A tale scopo fissiamo un punto $\xi$ e supponiamo $\bar{\xi} \in I(\xi)$. Consideriamo valori positivi di $\varepsilon$ tali che $I(\bar{\xi}, \varepsilon) \subset I(\xi)$, ed indichiamo con $\bar{t}$ e $\sigma(\bar{t}, \varepsilon)$ le proiezioni ortogonali di $\bar{\xi}$ ed $I(\bar{\xi}, \varepsilon)$ su $\Pi_{\bar{s}}$.

Si avrà :

$$
\begin{gathered}
\int_{I(\xi)-I(\bar{\xi}, \varepsilon)} \frac{\Phi(\eta-\bar{\xi})}{|\eta-\xi|^{m-1}} \varphi(\eta) d_{\eta} \sigma=\int_{\{|t|<d\}-\sigma(\bar{t}, \varepsilon)} \frac{\Phi_{k}(\xi, t-\bar{t}, 0)}{|t-\bar{t}|^{m-1}} \varphi_{k \xi \xi}(t) d t \\
+\int_{|| t|<d|-\sigma(\bar{t}, \varepsilon \mid} H_{k}(\xi, t, t)_{\varphi_{k \xi}(t)}(t)
\end{gathered}
$$

essendo $I(\xi) \subset A_{k}$ ed avendo posto

$$
H_{k}(\xi, t, \bar{t})=\frac{\Phi_{k}\left[\xi, t-\bar{t}, f_{k}(\xi, t)-f_{k}(\xi, \bar{t})\right]}{\left.\left.|| t-\left.\bar{t}\right|^{2}+\left[f_{k}(\xi, t)-f_{k l} \mid \xi, \bar{t}\right)\right]^{2}\right\}^{\frac{m-1}{2}}} J_{k}(\xi, t)-\frac{\Phi_{k}(\xi, t-\bar{t}, 0)}{|t-\bar{t}|^{m-1}}
$$

per la quale si trova

$$
\left|H_{k}(\xi, t, \bar{t})\right| \leq c \mid t-\bar{t}^{2-m}
$$

essendo $c$ una costante indipendente da $\xi$.

Detto ora $\xi^{*}$ un puntọ generico di $f(\bar{\xi}, \varepsilon)$ e $t^{*}$ la sua proiezione su $I_{\xi}$, indichiamo con $\Xi^{*}$ l'intersezione della semiretta $\overrightarrow{\vec{t} t^{*}}$ con l'ipersuperficie sferica di $\Pi_{\xi}$ col centro in $\bar{t}$ e raggio nguale ad uno e con

$$
\rho=p_{k}\left(\xi, \bar{t}, \varepsilon, \mathbf{E}^{*}\right)
$$

l'equazione in coordinate polari, eol polo in $\bar{t}$, della varietà $f \circ(\bar{t}, z)$; indichiamo inoltre con $\eta^{*}$ la proiezione ortogonale di $\xi^{*}$ su $\Pi_{\xi *}$ E facile vedere che

$$
\rho_{k}\left(\xi, \bar{t}, \varepsilon, \Xi^{*}\right)=\overline{\bar{\xi}^{*} \eta^{*}} \cos \Omega+\overline{\overline{\xi_{\eta}}} \overline{\eta^{*}} \cos \Psi
$$


$\Psi$ e $\Omega$ essendo i rispettivi angoli che gli assi $\bar{\xi} \eta^{*}, \overrightarrow{\eta^{*} \xi^{*}}$ formano con le proprie proiezioni su $\Pi_{\xi}$. Osservando che $\overline{\overline{\xi_{\eta}}}=\varepsilon$, che $\overline{\xi^{*} \eta^{*}}$ è infinitesimo di ordine superiore rispetto ad $\varepsilon$, che $\cos \Psi$ è una funzione di $\xi, \bar{t} \mathrm{e} \mathbf{\Xi}^{*}$, di classe $C^{(1)}$ rispetto a ciascuna delle variabili, si deduce il

Lema (3.1) - Esiste una funzione $\beta_{k}(\xi, \bar{t}, \Xi)$ di classe $C^{(1)}$ rispetto a $\xi, \bar{t}, t$ tale che, fissati $\xi, \bar{t}$ e $\Xi$, si abbia

$$
\lim _{\varepsilon \rightarrow \mathbf{0}} \log \left\{\frac{1}{\varepsilon} \rho_{k}(\xi, \vec{t}, \varepsilon, \Xi)\right\}=\beta_{k}(\xi, \bar{t}, \Xi)=\log \cos \Psi
$$

tale relazione sussistendo uniformemente rispetto a $\Xi$; la funzione $\beta_{k}$ in due $\Xi^{\prime}$ e $\Xi^{\prime \prime}$ qualunque simmetrici rispetto a $\bar{t}$ assume lo stesso valore.

Osserviamo che per il secondo integrale che figura nella (3.5) si ha

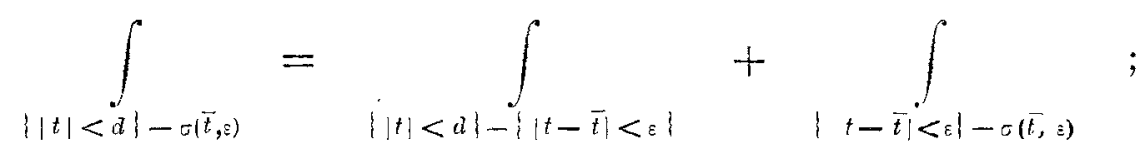

d'altra parte

$$
\begin{aligned}
& \int_{|| t-\bar{t}|<\varepsilon|-\sigma\left(\bar{t}_{, s}\right)} \frac{\Phi_{k}(\xi, \bar{t}-t, 0)}{|t-\bar{t}|^{m-1}} \varphi_{\Xi}(t) d t \\
& =\quad \int_{|| t-\bar{t} \mid<\varepsilon\}-\sigma(\bar{t}, \varepsilon)} \frac{\Phi_{k}(\xi, t-\bar{t}, 0)}{t-\left.\bar{t}\right|^{m-1}}[\varphi \varepsilon(t)-\varphi \xi(\bar{t})] d t \\
& +\varphi_{\varepsilon}(\bar{t}) \int_{|| t-\bar{t}|<\varepsilon|-\sigma\left(\bar{t} \_s\right)} \frac{\Phi_{l}(\xi, t-\bar{t}, 0)}{\mid t-\bar{t} m^{m-1}} d t .
\end{aligned}
$$

Poichè la $\varphi \in C^{(0, \alpha)}$ segue

$$
\begin{gathered}
\lim _{t \rightarrow 0} \int_{|| t-\bar{t} \mid<\varepsilon\}-\sigma(\bar{t}, \varepsilon)} \frac{\Phi_{k}(\xi, t-\bar{t}, 0)}{|t-\bar{t}|^{m-1}}\left[\rho_{\xi}(t)-\varphi_{s}(\bar{t})\right] d t=0 \\
\int_{|| t-\left.\bar{t}\right|_{<\varepsilon\}-\sigma(\bar{t}, \varepsilon)}} \frac{\Phi_{k}(\xi, t-\bar{t}, 0)}{|t-\bar{t}|^{m-1}} d t=\int_{\partial \mathbf{S}_{m-1}} \Phi_{k}(\xi, t-\bar{t}, 0) \log \left\{\frac{1}{\varepsilon} \rho_{k}(\xi, \bar{t}, \varepsilon, t-\bar{t})\right\} d_{t} \sigma .
\end{gathered}
$$


Sostituendo nella (3.5), passando al limite per $\varepsilon \rightarrow 0$ e tenendo presente la prima parte del lemma.(3.1), si stabilisce il

TeOREMA (3.2) - Fissato un punto $\xi \in A_{k}$, comunque si consideri un punto $\bar{\xi} \in I(\xi)$, nelle stesse ipotesi del teorema (3.1) si ha:

$$
\begin{aligned}
& \lim _{\varepsilon \rightarrow 0} \int_{\partial T-I(\bar{\xi}, \varepsilon)} \frac{\Phi(\eta-\bar{\xi})}{\mid \eta-\bar{\xi}_{\mid}^{m-1}} \varphi(\eta) d_{\eta} \sigma=\int_{\partial T-I(\xi)} \frac{\Phi(\eta-\bar{\xi})}{|\eta-\bar{\xi}|^{m-1}} \varphi(\eta) d_{\eta} \sigma \\
& +\varphi_{\xi}(t) \chi_{k}(\xi, \bar{t})+\int_{\{|t|<d\}}^{*} \frac{\Phi_{k}(\xi, t-\bar{t}, 0)}{|t-\bar{t}|^{m-1}} \varphi_{\xi}(t) d t \\
& +\int_{\{|t|<d\}} H_{k}(\xi, t, \bar{t}) \varphi \subseteq(t) d t
\end{aligned}
$$

avendo posto

$$
\begin{gathered}
\chi_{k}(\xi, \bar{t})=\int_{\{|t-\bar{t}|=1\}} \Phi_{k}(\xi, t-\bar{t}, 0) \beta_{k}(\xi, \bar{t}, t-\bar{t}) d t \sigma \\
\int_{\{|t|<d\}}^{*} \frac{\Phi_{k}(\xi, t-\bar{t}, 0)}{|t-\bar{t}|^{m-1}} \varphi_{\xi}(t) d t=\lim _{\varepsilon \rightarrow 0} \int_{\{|t|<d\}-\{|t-\bar{t}|<\varepsilon\}}[\cdots] d t .
\end{gathered}
$$

$\dot{\mathrm{E}}$ bene osservare che $\chi_{k}(\xi, 0)=0$. Si riconosce allora facilmente che la (3.12) per $\bar{t}=0$, si riduce alla (3.2). Dalla formula (3.12) si può dedurre il

Teorema (3.3) - Nelle ipotesi del teorema (3.1), posto

$$
\tilde{\varphi}=\int_{\partial T}^{*} \frac{\Phi(\eta-\xi)}{\left.|\eta| \xi\right|^{m-1}} \varphi(\eta) d_{n} \sigma=\lim _{\varepsilon \rightarrow 0} \int_{\partial T-I(\xi, \varepsilon)}[\ldots] d_{\eta} \sigma,
$$

si ha $\tilde{\varphi} \in C^{(0, x)}(\partial T)$ ed inoltre

$$
[\tilde{\varphi}]_{a} \leq c[\varphi]_{\alpha}
$$

essendo c una costante indipendente da $\varphi\left({ }^{10}\right)$.

(10) Questo viene chiamato, comunemente, teorema di GIRAUD, la sua dimostrazione, in condizioni più generali di quelle in cui noi ci siamo messi, può essere trovata in [9] Cap. I. 
TeOrema (3.4). - Se $\varphi \in L^{(2)}(\partial T)$ per quasi tutti $i$ punti vale la (3.12); indicato tale limite con $\tilde{\varphi}$, conformemente alla (3.14) si ha $\tilde{\varphi} \in L^{(2)}(\partial T)$

In effetti il ragionamento da noi fatto per stabilire la (3.12), ei dice che, se la $\varphi$ non è di classe $C^{(0, \alpha)}(\partial T)$, la stessa formula sussiste in ogni punto $\bar{\xi}$ (e quindi in $\bar{l})$, in cui vale la $(3.10)$, ha senso l'integrale

$$
\int_{\{|t|<d\}} H_{k}(\xi, t, \bar{t}) \varphi_{s}(t) d t
$$

ed esiste il limite a secondo membro della (3.13').

Osserviamo che, se $\varphi \in L^{(2)}(\mathfrak{C} T), \varphi \underline{s}(t)$ è di quadrato sommabile su $\{|t|<d\}$. Un teorema (11) di CaLderon e ZYGMUNd assicura che per quasi tutti $\mathrm{i}$ punti $\bar{t} \in\{|t|<d\}$ esiste il limite a primo membro della (3.13). In parti. colare ciò si avrà per quasi tutti i punti di Lesesgue della $\varphi_{s}(t)$, cioè per quasi tutti i punti, per cui si ha:

$$
\lim _{\varepsilon \rightarrow 0} \frac{1}{\varepsilon^{m-1}} \int_{\{|t-\bar{t}|<\varepsilon\}}\left|\varphi_{s}(t)-\varphi_{s}(t)\right| d t=0,
$$

o equivalentemente:

$$
\lim _{\varepsilon \rightarrow 0} \frac{1}{\varepsilon^{m s}-1} \int_{I(\xi, \xi)}|\varphi(\eta)-\varphi(\bar{\xi})| d_{r_{1}} \sigma=0 .
$$

Per dimostrare la prima parte dèl nostro asserto basterà quindi far vedere che se in un punto $\bar{t}$ è verificata la (3.17), vi è verificata anche la (3.10).

Ora un semplice calcolo mostra che il più piccolo valore per il cos $\Psi$ che figura nella (3.8) è $\frac{1}{J_{k}(\xi, \bar{t})}$. Di qui segue che è possibile determinare un $\varepsilon_{0}$ tale che per $0<\varepsilon<\varepsilon_{0}$ si abbia:

$$
|t-t| \geq \frac{\varepsilon}{2 J_{k}(\xi, \bar{t})}
$$

(11) Cfr. nota (4): si tratta, più precisamente del teorema $(2,1)$ di [5]. Rileviamo che il citato teorema è stabilito nella ipotesi che la densità è di potenza $p$-esima sommabile $(p>1)$. 
per ogni $t \in\{|t-\bar{t}|<\varepsilon\}-\sigma(\bar{t}, \varepsilon) ;$ per ogni $\varepsilon<\varepsilon_{0}$ si avrà allor’

$$
\begin{aligned}
& \left|\int_{|| t-i|<\varepsilon|-\sigma(\bar{t}, s)} \frac{\Phi_{k}(\xi, t-\bar{t}, 0)}{|t-\bar{t}| m-1}\left[\varphi_{\xi}(t)-\varphi_{\xi}(\bar{t})\right] d t\right| \\
& \leq \frac{c}{\varepsilon^{m-1}} \int_{|| t-\bar{t} \mid<\varepsilon\}-\sigma(\bar{t} \varepsilon),}\left|\varphi_{\xi}(t)-\varphi_{\xi}(\bar{t})\right| d t,
\end{aligned}
$$

da cui segue facilmente ciò che ci eravamo posto di dimostrare. La seconda parte del teorema ̀े conseguenza della formula (3.12) e del teorema di Caldorron e Zygmund.

Sia $\Sigma$ una porzione di $\partial T$ opportunamente piccola, $\Sigma_{\delta}$ una varieta parallela $\left({ }^{12}\right)$ a $\Sigma$ ed interna a $T,\left(\Sigma, \Sigma_{\delta}\right)$ lo strato determinato da $\Sigma$ e da $\Sigma_{\delta}$; per ogni $x \in\left(\Sigma, \Sigma_{\delta}\right)$ passa una ed una solo l'etta normale a $\Sigma$. Di conseguenza un tale punto è completamente determinato dalla sua proiezione ortogonale $\xi$ su $\Sigma$ e dalla distanza $\rho$ da essa. Consideriamo la funzione

$$
v(\xi, \rho)=v(x)=\int_{|1 y-\xi|<d\} \cap\{y \cdot n(\xi)=0\}} \frac{\Phi(y-x)}{|y-x|^{m-1}} d_{y} \sigma .
$$

Sussiste il seguente

Teorema (3.5) - Te $\Phi$ verifica le ipotesi $\left.I_{4}\right)$ e $\left.I_{5}\right)$, la v( $(\xi$, ol al tendere di $\rho$ a zero converge uniformemente rispetto a $\xi \in \Sigma$ e se $\Sigma \subset A_{k}$ si ha:

$$
\lim _{i \rightarrow 0} v(\xi, \rho)=\int_{0}^{\frac{\pi}{2}} \frac{\operatorname{sen}^{m-2} \alpha}{\cos \alpha} d x \int_{\{|t|=1\}} \Phi_{k}(\xi, t \operatorname{sen} \alpha,-\cos \alpha) d_{t} \sigma .
$$

Nelle ipotesi poste introdotto un sistema di coordinate polari $r$, $\Theta$ nel piano $\Pi_{\xi}$ col polo in $\xi$, si avrà :

e quindi

$$
|y-\xi|=\sqrt{r^{2}+\rho^{2}} \quad d \sigma=r^{m-2} d r d \sigma_{m-2}
$$

$$
v(\xi, \rho)=\int_{0}^{d} \frac{r^{m-2}}{\left(r^{2}+\rho^{2}\right)^{\frac{m-1}{2}}} d r \int_{\{|t|=1\}} \Phi_{k}(\xi, r t,-\rho) d_{t} \sigma_{m-2}
$$

${ }^{(12)}$ L'ipotesi che $T$ è di classe $\mathbf{A}^{(2)}$ interviene soprattutto in questo punto. 
essendo la $\Phi$ positivamente omogenea di grado zero, tale risulterà la funzione $\Phi_{k}(\xi, r t,-\rho)$ rispetto alle variabili $\left(r_{1}, \ldots, r \tau_{m-1},-\rho\right)$; eseguendo allora il cambiamento di variabile

$$
r=p t g \alpha
$$

si ottiene

$$
v(\xi ; \rho)=\int_{0}^{\operatorname{arctg} \frac{d}{b}} \frac{\operatorname{sen}^{m-2} \alpha}{\cos \alpha} d \alpha \int_{|| t \mid=1\}} \Phi_{k}(\xi, t \operatorname{sen} \alpha,-\cos \alpha) d_{t} \sigma .
$$

Osservando che

$$
\int_{\{\mid t\}=1\}} \Phi_{k}(\xi, t, 0) d_{t} \sigma_{m-2}=0
$$

e sfruttando l'ipotesi $I_{5}$, si deduce facilmente il nostro asserto.

Il limite a secondo membro della (3.19) sarà indicato col simbolo $v^{+}(\xi)$; analogamente indicheremo con $v-(\xi)$ il limite della funzione $v(x)$ se $x \in \mathfrak{C} T$ e si fa tendere $\varphi(=\overline{x \xi}$ a zero. Con analogo procedimento si trova:

$$
v-(\xi)=\int_{0}^{\frac{\pi}{2}} \frac{\operatorname{sen}^{m-2} \alpha}{\cos \alpha} d \alpha \int_{\{|t|=1\}} \Phi_{k}(\xi, t \operatorname{sen} \alpha, \cos \alpha) d_{t} \sigma
$$

Ci interessa, ora, mettere in evidenza, come conseguenza delle formule (3.19) e $\left(3.19^{\prime}\right)$, il

Teorema (3.6) - Se $\Phi$ verifica le ipotesi $\left.\mathrm{I}_{4}\right)$ e $\mathrm{I}_{5}$ l, ed è dispari $\Phi(x)=-\Phi(-x)$, per ogni $\xi \in \Sigma$ si ha:

$$
v^{+}(\xi)=-v^{-}(\xi)
$$

Infine con procedimento ormai classico, si dimostra il

TEOREMA $(3.7)$ - Fissato il punto $\xi \in \Sigma$ ed una semiretta $l_{\xi}$ non tangente $a \mathrm{\Sigma}$ in $\xi$ e divetta verso l'interno [l'esterno] di $T$, si ha

$$
\lim _{\substack{x \rightarrow \xi \\ x \in u(\xi)}} v(x)=v^{+}(\eta) \quad\left[v^{-}(\eta)\right]
$$


Facciamo ora vedere che si ha

$$
\lim _{\substack{x \rightarrow \xi \\ x \in T-\partial T}} w(x)=v^{+}(\xi) \varphi(\xi)+\int_{\partial T}^{*} \frac{\Phi(\eta-\xi)}{\eta-\left.\xi\right|^{m-1}} \varphi(\eta) d_{\eta_{i} \sigma}
$$

uniformemente rispetto a $\xi$ su $\partial T$.

Infatti, fissato $\xi$, determiniamo una regione $\Sigma$, contenente $\xi$ e contenuta in un aperto $A_{k}$ del ricoprimento, in modo che si possa considerare lo strato $\left(\Sigma, \Sigma_{\delta}\right)$ di cui si siamo serviti per stabilire il teorema (3.4).

Posto :

$$
\begin{aligned}
H_{k}^{\prime}(\xi, t, \rho) & =\frac{\Phi_{k}\left(\xi, t, f_{k}(\xi, t)-\rho\right)}{\left[|t|^{2}+\left(f_{k}(\xi, t)-\rho\right)^{2}\right]^{m-1}} J_{k}(\xi, t) \\
& -\frac{\Phi_{k}(\xi, t,-\rho)}{\left[|t|^{2}+\rho \rho^{2}\right]^{\frac{m-1}{2}}},
\end{aligned}
$$

si trova:

$$
\int_{I(\xi)} \frac{\Phi(\eta-x)}{|\eta-x|^{m-1}} d_{\eta \eta} \sigma=v(\xi, \rho)+\int_{|| t \mid<\alpha\}} H_{k l}^{\prime}(\xi, t, \rho) d t
$$

inoltre con semplice procedimento si riconosce che il nucleo $H_{k}^{\prime}(\xi, t, \rho)$ si decompone nella somma di un numero finito di nuclei del tipo $O\left(\rho^{2} \cdot \mid t_{1}-r\right)$ con $\gamma$ e $\beta$ verificanti la condizione

$$
\beta+m-1>\gamma
$$

e ciò assicura ohe $\left({ }^{13}\right)$

$$
\lim _{\rho \rightarrow 0} \int_{\{|t|<d\}} H_{k}^{\prime}(\xi, t, \rho) d t=\int_{\{|t|<d\}} H_{k}(\xi, t) d t .
$$

Per dimostrare la (3.22) basta scrivere $w(x)$ come segue

$$
\begin{aligned}
w(x) & =\int_{\partial T-I(x)} \frac{\Phi(\eta-x)}{|\eta-x|^{m-1}} \varphi(\eta) d_{\eta} \sigma+\int_{I(\xi)} \frac{\Phi(\eta-x)}{|\eta-x|^{m-1}}[\varphi(\eta)-\varphi(\xi)] d d_{\eta} \sigma \\
& +\varphi(\xi) \int_{I(\xi)} \frac{\Phi(\eta-x)}{|\eta-x|^{m-1}} d_{\eta_{1} \sigma}
\end{aligned}
$$

(13) Cfr. [20] Cap. II, il lemma del n. 14. 
tener presente la $(3,23)$, passare al limite ed infine ricordare $1 a, 3,2 ;$. Analoga formula sussiste se si prende $x \in \mathfrak{C} T$.

In forza della (3.22) e della seconda parte del teorema (3.3) si stabilisce facilmente il

'Твоннма (3.8) - $\left({ }^{14}\right)$ Se $\varphi \in O^{(0, \alpha)}(\partial T)$ e $\Phi$ verifica le ipotesi $\left.\mathrm{I}_{4}\right)$ e $\left.\mathrm{I}_{5}\right)$, allora $x \in C^{(0, \alpha)}(T)\left[C^{(0, x)}(\mathfrak{C} T+\partial T)\right]$. Posto

$$
w^{+}(\xi)=\lim _{\substack{x \rightarrow \xi \\ x \in T-\partial T}} w(x) \quad w^{-}(\xi)=\lim _{\substack{x \rightarrow \sum_{x} \\ x \in \mathfrak{C}_{T}}} w(x)
$$

si ha

$$
w=v \dot{=}(\xi) \varphi(\xi)+\int_{\partial T}^{*} \frac{\Phi(\eta-\xi)}{|\eta-\xi|^{m-1}} \varphi(\eta) d d_{r_{1}} \sigma .
$$

Sussiste il

TeOREMa (3.9) - Se $\varphi \in L^{(2)}(\mathrm{aT}) i$ limiti $w^{+}$e w-, fatti per $x \rightarrow \xi$ secondo una direzione $l_{5}$ non tangente $a \partial T$ in $\xi$, esistono per quasi tutti $i$ punti $\xi \in \partial T$, sono dati quasi ovunque su $\partial T$ dalla formula (3.25), e definiscono due funzioni di classe $L^{(i)}(\partial T)$.

Per dimostrarlo osserviamo che, se in un punto $\xi \in \partial T$ esiste il limite a primo membro della (3.2), esiste anche il

$$
\lim _{\varepsilon \rightarrow 0} \int_{I(\xi)-I(\xi ; \xi)} \frac{\Phi(\eta-\xi)}{|\eta-\xi|^{m-1}}[\varphi(\eta)-\varphi(\xi)] d_{r \eta} \sigma,
$$

come si ricava dalla (3.4). Tenendo presente la $(3.24)$ ed il teorema (3.4), si riconosce che, per dimostrare il nostro asserto, baster't dimostrare che in ogni punto in cui esiste il limite (3.26) si ha:

$$
\begin{aligned}
& \lim _{\substack{x \rightarrow l_{\xi} \\
x \in l_{\xi}}} \int_{I(\xi)} \frac{\Phi(\eta-x)}{\mid \eta-x^{\mid m-1}}[\varphi(\eta)-\varphi(\xi)] d_{\eta} \sigma \\
= & \lim _{\varepsilon \rightarrow 0} \int_{I(\xi)-I(\xi, \varepsilon)} \frac{\Phi(\eta-\xi)}{\mid \eta-\xi^{m-1}}[\varphi(\eta)-\varphi(\xi)] d_{\eta} \sigma .
\end{aligned}
$$

(14) In sostanza questo teorema è analogo al teorema $(3,1)$ di [1]. 
Che sussista poi una tale eguaglianza è conseguenza immediata del fatto che in ogni punto di LeBesgue $\xi(\in \partial T)$ della $\varphi$, si ha $\left({ }^{15}\right)$ :

$$
\begin{aligned}
& \lim _{\substack{x \rightarrow \sum_{x \rightarrow \xi} \\
I(\xi)-I(\xi,|x-\xi|)}}\left[\frac{\Phi(\eta-x)}{\mid \eta-x^{\mid m-1}}-\frac{\Phi(\eta-\xi)}{|\eta-x|^{m-1}}\right][\varphi(\eta)-\varphi(\xi)] d_{\eta} \sigma=0 \\
& \lim _{\substack{x \rightarrow \xi_{0} \\
x \in l_{I} I(\xi,}} \int_{x-\xi \mid)} \frac{\Phi(\eta-x)}{|\eta-x|^{m-1}}[\varphi(\eta)-\varphi(\xi)] d_{\eta} \sigma=0
\end{aligned}
$$

Per stabilire la (3.27) introduciamo il nucleo così definito

$$
K(x, \eta)=\left\{\begin{array}{lr}
\frac{\Phi(\eta-x)}{|\eta-x|^{m-1}-\frac{\Phi(\eta-\xi)}{|\eta-\xi|^{m-1}}} & \text { per } \eta \notin I(\xi,|x-\xi|) \\
0 & , \eta \eta \in I(\xi,|x-\xi|)
\end{array}\right.
$$

Per l'ipotesi $I_{5}$ ) si stabilisce facilmente che esiste una costante $c$ tale che si abbia:

$$
\begin{aligned}
& \left|\frac{\Phi(\eta-x)}{|\eta-x|^{m-1}}-\frac{\Phi(\eta-\xi)}{|\eta-x|^{m-1}}\right| \leq c\left[\frac{1}{|\eta-x|^{m-2}}|| \frac{1}{|\eta-x|}-\frac{1}{|\eta-\xi|} \mid\right. \\
+ & \left.\left.\frac{|x-\xi|}{|\eta-\xi|}\right\}+\left|\frac{1}{|\eta-x|^{m-1}}-\frac{1}{|\eta-\xi|^{m-1}}\right|\right]
\end{aligned}
$$

Da ciò sfruttando il fatto che $x$ lo si prende su una retta non tangente a $\partial T$ in $\xi$ e che $T \dot{e}$ di classe $A^{(2)}$, si stabilisce con procedimenti elemen. tari che

$$
K(x, \eta)=0\left(x-\xi|\cdot| \eta-\left.x\right|^{-m}\right)\left({ }^{16}\right) .
$$

D'altra parte, poichè l'integrale che figura nella (3.27) si può scrivere:

$$
\int_{I(\xi)} K(x, \eta)(\varphi(\eta)-\varphi(\xi)) d m_{n} \sigma,
$$

(15) Vogliamo rilevare che questa dimostrazione è suggerita da quella del teorema IX del Cap, I di [8].

(15) Questa formula si dimostra facilmente nel caso in cui la porzione $I(E, d)$ di $\partial T$ ̀̀ piana. Infatti detta $i_{5}$ la retta seconcio cui si fa tendere $x$ a $\overline{5}$ ed $\omega$ l'angolo che questa retta forma con la sua proiezione su $\mathrm{II}_{\zeta}$ si stabilisce facilmente che per ogni

$$
\eta \in I(\xi, d)-I(\xi,|x-\xi|)
$$

si ha $2|\eta-\xi| \geq|\eta-x| \geq$ sen $\omega|\eta-\xi|$; sostituenda nella $(3,29)$ e tenendo presente che ||$\eta-x|-| \eta-\xi|\leq| x-\xi \mid$ si trova subito la $(3,30)$. 
segue che, se $\xi$ è un punto di LEBEsGUe della $\varphi$, si può passare al limite sotto il segno di integrale per $x \rightarrow \xi$, e ciò dà proprio la (3.27).

Per quanto riguarda la (3.28) basta osservare che esiste una costante $c$ tale che per $\eta \in I(\xi,|x-\xi|)$ si abbia:

$$
|\eta-x \geq c| x-\xi \mid,
$$

da cui segue che l'integrale che figura nella (3.28) si può maggiorare con l'altro

$$
\frac{1}{|x-\xi|^{m-1}} \int_{I(\xi, \mid x-\xi)}|\varphi(\eta)-\varphi(\xi)| d \sigma
$$

moltiplicato per una opportuna costante. Se ne deduce la (3.28) in ogni punto di LESBEgue della $\varphi$, c.v.d.

4). Richiami sulla teoria degli operatori integrali singolari. In questo paragrafo richiameremo prima di tutto alcuni risultati dovuti a CALDERON Zyguund [6], e ce ne varremo per dimostrare un facile teorema $[(4.2)] \mathrm{di}$ cui dovremo servirci in seguito. Richiameremo poi alcuni teoremi che sono da attribuirsi a S. G. MiHLIN [15], relativi agli integrali singolari estesi all'intero spazio. Dimostreremo, quindi, un teorema $[(4,6)]$ che permette di dominare la teoria delle equazioni integrali, in cui intervengono i suddetti integrali. Per questa dimostrazione ci varremo dei citati risultati della memoria [6] e della teoria delle trasformazioni riducibili in uno spazio di Banach. Infine indicheremo come questi risultati si possono generalizzare per il caso di integrali estesi ad una varietà.

Siano:

$\mathbb{R}\left(E_{k}\right)$ la classe delle funzioni $f \in L^{(2)}\left(E_{k}\right)$, ciascuna delle quali dipende soltanto da $|x|$.

$\mathfrak{C}\left(E_{k}\right)$ quella delle funzioni $f \in C^{\infty}\left(E_{k}\right)$ tali che, comunque si fissi un intero positivo $n$, la $f$ ed ogni sua derivata siano per $|x| \rightarrow \infty$ infinitesime di ordine superiore a $|x|^{-n}$.

$\mathbb{C}^{\mathbb{N}}\left(E_{k}\right)$ il sottoinsieme di $\mathcal{C}$ delle $f$, tali che

$$
\int_{\mathbf{E}_{k}} f(x) g(x) d x=0
$$

per ogni $g \in \mathbb{R}\left(E_{k}\right)$ 。

$\mathbb{D}^{(\beta)}\left(E_{k c}\right)$ quella delle funzioni $f \in C^{\infty}\left(E_{k}-\{O\}\right)$, positivamente omogenee 
di grado $-\beta$ che verificano la condizione

$$
\int_{\partial \mathbf{\Omega}_{k}} \Phi(x) d \sigma=0 \text {. }
$$

Per ogni $f$ definita in $E_{k}$, detti $\lambda$ e $\mu$ due numeri reali non negativi $(\mu \leq+\infty)$, poniamo

$$
\begin{array}{ll}
f_{\lambda}^{\mu}=f(x) \quad \text { se } \lambda \leq|x|<\mu ; \quad f_{\lambda}^{\mu}=0 \text { per gli altri } x . \\
f^{\mu}=f_{0}^{\mu} ; \quad f_{\lambda}=f_{\lambda}^{+\infty}
\end{array}
$$

Se $f \in L^{(1)}\left(E_{k}\right)$ indichiamo con $\mathscr{F}(f)$ o con $\tilde{f}$ la sua trasformata di FOURIER.

Se $\Phi \in \mathbb{D}^{(k)}\left(E_{k}\right)$, poniamo:

$$
\begin{aligned}
& \mathscr{F}\left(\Phi_{\lambda}\right)=\lim _{\mu \rightarrow+\infty} \mathscr{F}\left(\Phi_{\lambda}^{\mu}\right) \\
& \mathscr{F}(\Phi)=\lim _{\lambda \rightarrow 0} \mathscr{F}\left(\Phi_{\lambda}\right)\left({ }^{17}\right) .
\end{aligned}
$$

Si trova $\left({ }^{18}\right)$

$$
\tilde{\Phi}(y)=\mathscr{F}(\Phi)=\int_{\partial \Omega} \Phi(x)\left\{\log \frac{1}{|\cos x \tilde{o y}|}+i \frac{\pi}{2} s g(\cos x \hat{o} y)\right\} d \sigma\left({ }^{19}\right)
$$

Infatti usando le coordinate polari si ha:

$$
\begin{gathered}
\mathscr{F}\left(\Phi_{\lambda}^{\mu}\right)=\int_{E_{k}} \Phi_{\lambda}^{\mu}(x) e^{i x y} d x= \\
=\int_{\partial \Omega_{k}} \Phi(x) d_{x} \sigma \int_{\lambda}^{\mu} \frac{1}{\rho} e^{i \rho|y| \cos x \hat{o} y} d \rho,
\end{gathered}
$$

(17) Intenderemo che la (4.2) e (4.2') definiscono $\mathscr{F}\left(\Phi^{\mu}\right)$ ed $\mathscr{F}(\Phi)$ quasi ovunque in $E_{k}$.

(18) Cfr. [6].

(19) Con sg abbiamo indicato la funzione definita sull'insieme dei numeri reali che assume il valore 1 in ogni numero non negativo ed il valore -1 in ogni numero negativo. 
da cui per $|y|>0$ e $\cos x \hat{y} y \neq 0$, posto

$$
\rho ; y|| \cos x \widehat{O} y=t \quad \lambda\left|y_{1}\right| \cos \hat{x o y}|=r ; \mu| y|\cos \hat{x o y}|=R
$$

si ha:

$$
\begin{aligned}
& \int_{i}^{1} \frac{1}{\rho} e^{i} y^{\prime} \cos x \hat{\theta} y d \rho=\int_{r}^{1} \frac{\cos t-1}{t} d t+\int_{i}^{R} \frac{\cos t}{t} d t \\
& +i s g(\cos x \bar{o} y) \int_{r}^{R} \operatorname{sen} t d t+l g \frac{1}{r} \text {. }
\end{aligned}
$$

Sostituiamo nella (4.4); tenendo presente la (4.1), si ottiene:

$$
\begin{aligned}
\tilde{F}(\Phi) & =\int_{\partial \Omega_{k r}} \Phi\left\{\int_{r}^{1} \frac{\cos t-1}{t} d t+\int_{1}^{R} \frac{\cos t}{t} d t+i s g(\cos x \tilde{o} y) \cdot \int_{r}^{R} \frac{\operatorname{sen} t}{t} d t\right. \\
& \left.+\log \frac{1}{|\cos x \bar{x} y|}\right\} d_{x} \sigma .
\end{aligned}
$$

Poichè la fungione integranda in quest' ultima formula è maggiorata dalla funzione

$$
\log \frac{c}{|\cos x \hat{y} y|}
$$

con $c$ opportunamente scelta, segue che si può eseguire il passaggio al limite sotto il segno di integrale per $\mu \rightarrow \infty$ e $\lambda \rightarrow 0$; così facendo si trova la (4.3).

Si dimostrano le seguenti proposizioni:

$$
\begin{array}{lll}
\text { Se } f \in \mathbb{C} & \text { anche } & \mathscr{F}(f) \in \mathbb{C} \\
\text { Se } f \in \mathbb{R} & \text { anche } & \mathscr{F}(f) \in \mathbb{R} \\
\text { Se } f \in \mathbb{C}^{\mathbb{R}} & \text { si ha } & f(O)=0 \\
\text { Se } f \in \mathbb{C}^{\mathbb{R}} & \text { anche } & \mathscr{F}(f) \in \mathbb{C}^{\mathbb{R}} \\
\text { Se } f \in \mathbb{C}^{\mathbb{R}}, & \text { posto } & \\
& \Phi(x)=\int_{0}^{\infty} f(\lambda x) \lambda^{\beta-1} d \lambda
\end{array}
$$


si ha $\Phi \in \mathbb{D}^{(\beta)}\left(E_{k}\right)$; viceversa assegnata una $\Phi \in \mathbb{p}^{(\beta)}\left(E_{k}\right)$. detta $\rho(t)$ una fun. zione di classe $C^{\infty}([0 .+\infty])$ ed a supporto compatto contenuto in $(0,+\infty)$, tale che

$$
\int_{0}^{\infty} \frac{\rho(t)}{t} d l=1
$$

posto

$$
f(x)=\Phi(x) \rho(|x|)
$$

si ha che $f \in \mathbb{C}^{\mathbb{1 R}}$, e sussiste la (4.5).

Se $\Phi \in \mathbb{P}^{(k)}\left(E_{k}\right)$ posto conformemente alla $(4.5)$

$$
\Phi(x)=\int_{0}^{\infty} f(\lambda x) \lambda^{k-1} d \lambda
$$

si $h a$

$$
\mathcal{F}(\Phi)=\int_{0}^{\infty} \tilde{f}(\lambda y) \lambda^{-1} d \lambda .
$$

Da tali proposizioni si deduce facilmente il

Teorema $(4.1)$ - Se $\Phi \in \mathbb{D}^{(k)}\left(E_{k}\right)$, si ha $\mathscr{F}(\Phi) \in \mathbb{P}^{(0)}\left(E_{k}\right)$; comunque si prenda una $\Psi \in \mathbb{D}^{(0)}\left(E_{k}\right)$ esiste una $\Phi \in \mathbb{D}^{(k)}\left(E_{k}\right)$ tale che si abbia:

$$
\mathscr{F}(\Phi)=\Psi
$$

Questo teorema è dimostrato in [7]. Vogliamo mettere in risalto come se ne stabilisce la seconda parte per dedurre un corollario che avrà un ruolo molto importante nella nostra trattazione. Sia quindi $\Psi \in \mathbb{D}^{(0)}\left(E_{k}\right)$; conformemente alla $(4.5)$ si avrà

$$
\Psi(x)=\int_{0}^{\infty} f\left(\lambda x \mid \lambda^{-1} d \lambda,\right.
$$

avendo posto

$$
f(x)=\Psi(x) \rho(|x|) .
$$

Considerando la funzione

la funzione

$$
g=g^{-1}(f)
$$

$$
\Phi=\int_{0}^{\infty} g(\lambda x) \lambda^{k-1} d \lambda
$$


i tale che

$$
\widetilde{F}(\Phi)=\Psi \text {. }
$$

Si deduce di qui il

Teonema $(4.2)-S e b=\left(\left(b^{(1)}(\xi, \tau), \ldots . b^{(N)}(\xi, \tau) \|\right.\right.$ per ogni fissalo $\tau \neq 0 \dot{e}$, rispetto a $\xi$, un elemento dello spazio $C^{(0, \alpha)}\left\{\{\Gamma\}_{A}\right\}$ e ciascuna delle funzioni $b_{l}{ }^{(h)}(\xi, \tau)$ per ogni $\xi \in A_{h}$ è, come funzione di $\tau$, di olasse $\mathbb{P}^{(k)}\left(E_{k}\right)$, posto

$$
\left.\tilde{b}_{l}^{(h)}(\xi, \tau)=\underset{t}{\mathcal{F}}\left(b_{l}(h) \xi \xi, l\right)\right)
$$

si ha che

$$
\tilde{b}(\xi, \tau)=\left(\left(\tilde{b}^{(1)}(\xi, \tau) \ldots, \tilde{b}^{(N)}(\xi, \tau)\right)\right)
$$

per ogni fissato $\tau$ è ancora un elemento di $C^{(0, \alpha)}\left(\{\mathbf{\Gamma}\}_{A}\right)$.

Indichiamo con $\mathfrak{A}_{0}$ la classe delle trasformazioni integrali del tipo

$$
\tau(\varphi)=\int_{E_{k}} L(x, y) \varphi(y) d y
$$

essendo $L(x, y)$ di classe $N^{(\beta)}$; con $\mathcal{H}$ la classe delle trasformazioni del tipo

$$
\mathscr{X}(\varphi)=f_{0}(x) \varphi(x)+\int_{E_{k}}^{*} \frac{f(x \cdot y-x)}{\mid y-x^{k}} \varphi(y) d y+\tau(\varphi)
$$

definite in $L^{(2)}\left(E_{k}\right)$ supponendo $\tau \in \mathfrak{Z}_{0}, f(x, z)$ per ogni fissato $x \in E_{k}$ come funzione di $z$ di classe $\mathbb{P}^{(0)}\left(E_{k}\right)$ e come funzione di $x$ sia di classe $C^{(0, \alpha)}\left(E_{k}\right)$ uniformemente rispetto a $z \in \partial \Omega_{m}$.

Sviluppata la $f$ in serie di funzioni ultrasferiche su $\Omega_{k}\left(^{-0}\right)$ :

$$
f(x, y)=\Sigma f_{r s}(x) Y_{2 \cdot s}\left(\frac{y}{|y|}\right)|y|^{-k}
$$

dicesi simbolo della trasformazione $\mathfrak{A}$ la funzione

$$
\sigma_{A}(x, y)=f_{0}(x)+\Sigma f_{r s}(x) \gamma_{r} Y_{r s}\left(\frac{y}{|y|}\right)
$$

(20) Per questo sviluppo ei sembra più comodo rinviare alla memoria [6] di cui man. terremo qui le notazioni. 
essendo

$$
\left.\gamma_{r}=i^{r} \pi^{\frac{k}{2}} \Gamma\left(\frac{r}{2}\right) /\left.\Gamma\left(\frac{r+k}{2}\right)\right|^{21}\right)
$$

Nelle ipotesi in cui ci siamo posti, la serie a secondo membro della (4.9) converge uniformenente $\left({ }^{22}\right)$ rispetto ad $y$ su $\partial \Omega_{k}$ per ogni fissato $x$. Da una proprietì della trasformata di Fourier delle $Y_{{ }_{s}}\left({ }^{23}\right)$ si deduce

$$
\sigma_{\mathfrak{Q}}=f_{0}(x)+\underset{y}{\mathfrak{F}}\left(\frac{f(x, y)}{|y|^{k}}\right)
$$

avendo $\mathscr{F}$ il significato che le abbiamo dato mediante la $\left(4.2^{\prime}\right)$.

Il simbolo dell'operatore identità è per definizione l'unità, quello di ogni trasformazione appartenente alla classe $\mathfrak{F}_{0}$ è lo zero.

TeOREMa (4.3) - Se due trasformazioni della classe $\mathfrak{z}$ hanno lo stesso simbolo, la loro differenza appartiene ad $\mathfrak{F}_{0}$.

TeORema (4.4) - Il prodotto (di composizione) di due trasformazioni della classe $\mathbf{Z}$, appartiene ad $\mathbf{Z}$ ed ha per simbolo il prodotto dei simboli $\left({ }^{24}\right)$.

Teorema (4.5) - Se la trasformazione $\mathfrak{A} \in \mathfrak{Z}$ ha per simbolo la funzione $\sigma_{\mathfrak{Q}}(\xi, \tau)$, la trasformazione aggiunta di $\mathfrak{A}$ appartiene ancora ad $\mathfrak{A}$, ed ha per simbolo la funzione complessa coniugata di $\sigma_{\mathfrak{Q}}(\xi, \tau)$.

In virtù di queste proposizioni si può risolvere il problema della riduzione di una trasformazione $\mathfrak{A} \in \mathfrak{Z}$. Per determinare una trasformazione $\mathfrak{A}^{\prime} \in \mathfrak{A}$ in modo che si abbia:

$$
\mathfrak{A}^{\prime} \mathfrak{Q}=\mathfrak{d}+\mathfrak{F}
$$

essendo $\mathfrak{J}$ l'identità ed $\mathscr{F}$ una trasformazione completamente continua in $L^{(2)}\left(E_{k}\right)$, cominciamo ad osservare che la (4.11) in virtù del teorema (4.3)

$\left.1^{21}\right)$ Soltanto in questa formula $\Gamma$ indica la gamma euleriana.

(22) Cfr. [15] Cap. II $\$ 20$.

(23) La citata proprietà assicura la trasformata di Fourier della funzione $y^{-k} Y_{r_{s}}\left(\frac{y}{|y|}\right)$ è uguale a $\gamma_{r} \quad Y_{r s}\left(\frac{y}{|y|}\right)$, cfr. [3]. La formula (t.10) per $k>2$ è stata messa in evidenza esplicitamente da A. P. CALDERON e A. ZYGMUND in [6]; è stata adoperata efficacemente da Mihlin in [18].

(24) Questo teorema nel caso in cui le $f$ sono indipendenti da $x$ è conseguenza di una ben nota proprietè della trasformata di Fourier del prodotto di composizione cfr. [7]; nel caso generale è stato dimostrato da S. G. MIHLIN in [16]. 
implica

$$
\sigma \mathfrak{Q}^{\prime} \cdot \sigma \mathfrak{Q} \equiv 1
$$

Supponiamo $\sigma_{\mathfrak{g}}(x, \tau) \neq 0$. per ogni $x \in E_{k}$ e $\tau \in \partial \Omega_{k}$.

Posto:

$$
g(x)=\frac{1}{\operatorname{mis} \partial \Omega_{k}} \int_{\partial \Omega_{k}}\left[\sigma_{A}(x, \tau)\right]^{-1} d \sigma
$$

e

$$
\left[\sigma_{A}(x, \tau)\right]^{-1}-g(x)=\Psi(x, \tau)
$$

Si ricava facilmente che $\Psi(x, \tau)$ per ogni fissato $x$ appartiene alla classe $\mathbb{0}^{(0)}\left(E_{k}\right)$.

Per il teorema (4.1) esiste una funzione $\Phi(x, z)$ tale che si abbia:

$$
\underset{\xi}{\mathscr{F}}(\Phi(x, z))=\Psi(x, y)
$$

(per costruire la $\Phi$, basta tener presente la proposizione 4.VI). Posto

$$
\mathfrak{d}_{1}(\varphi)=g(x) \varphi(x)+\int_{E_{k}} \Phi(x, y-x) \varphi(y) d_{y} \sigma
$$

per essere

$$
\sigma_{\mathfrak{A}_{1}}{ }^{\mathfrak{d}} \equiv 1
$$

si deduce in virtù del teorema (4.3)

$$
\mathfrak{A}_{1} \mathfrak{a}=\mathfrak{J}+\mathfrak{C}
$$

essendo $\tau$ una trasformazione appartenente alla classe $\mathfrak{F}_{0}$. Notiamo ora che esiste certamente un intero positivo $\vee$ tale $\tau^{\nu}$ è completamente con. tinua $\left({ }^{25}\right)$. È facile allora verificare che la trasformazione

$$
\mathfrak{U}^{\prime}=\left(\mathfrak{g}-\tau+\tau^{2}+\ldots+(-1)^{\nu-1} \tau^{\nu-1}\right) \mathfrak{d}_{1}
$$

è riducente $\mathfrak{A}$ nel senso precisato.

Applicando i teoremi (2.5) e (4.5), si deduce facilmente il

(25) A tale scopo basta tener presente un teorema fondamentale sul produtto in composizione di nuelei di elasse $N(\beta)$, cfr., per esempio, [20], cap. II. 
Thorema (4.6) - Se $\sigma_{\mathfrak{Q}}$ non è mai nullo, la trasformazione $A$ e la sua aggiunta sono a codominio chiuso ed ammettono entrambe un numero finito di autosoluzioni linearmente indipendenti.

Questa impostazione si estende $\left({ }^{26}\right)$ senza difficoltà agli operatori veltoriali del tipo:

$$
\sum_{k=1}^{n} \mathfrak{V}_{i k}\left(\varphi_{k}\right)=\varphi^{\prime}, \quad i=1, \ldots, n
$$

con $\mathfrak{Q}_{i k} \in \mathfrak{A}$ e $\left(\varphi_{1}, \ldots, \varphi_{n}\right) \in\left[L^{(2)}\left(E_{k}\right)\right]^{n}$. Ad un tale operatori si associa per per simbolo la matrice

$$
\left\|\sigma_{i k}\right\|
$$

avendo indicato con $\sigma_{i k}$ il simbolo della trasformazione $\mathfrak{A}_{i k}$. All'operatore identità si associa come simbolo la matrice $\left.\left.\left\|\delta_{, s}\right\|\right|^{27}\right)$. Il ruolo del simbolo (matrice simbolica) nel problema della riduzione si comprende attraverso i teoremi analoghi ai teoremi $(4.3),(4.4),(4.5)$.

Ci proponiamo ora di far vedere come queste considerazioni si possono estendere al caso in cui gli integrali a valor principale sono estesi a $\partial T$. Ciò si consegue però valendosi anche di alcuni procedimenti seguiti da Giradd nei casi particolari da lui considerati in occasione di sue ricerche sul problema di derivata obliqua.

A tale scopo consideriamo la classe $\mathfrak{G}$ delle trasformazioni del tipo

$$
\mathfrak{A}(\varphi)=a_{0}(\xi) \varphi(\xi)+\int_{\partial T}^{*} \frac{G(\xi, \eta)}{|\eta-\xi|^{m-1}} \varphi(\eta) d_{n} \sigma
$$

supponendo che se $\xi \in A_{h}$, rispetto al sistema $\left[\xi, \tau_{1}, \ldots, \tau_{m-1}, \tau_{m}\right]_{h}$, la funzione $G$ si possa decomporre nel seguente modo:

$$
\frac{G(\xi, \eta)}{|\eta-\xi|^{m-1}}=\frac{G_{h}(\xi, t)}{|t|^{m-1}}+G_{h}^{\prime}(\xi, \eta)
$$

essendo $G_{h}(\xi, t)$ rispetto a $t$, per ogni fissato $\xi \in A_{h}$, di classe $\mathbb{D}^{(0)}\left(E_{m-1}\right)$ e $G_{h}^{\prime}(\xi, \eta)$ di classe $N^{(\beta)}\left(A_{h}\right)$; se $\xi \in A_{h} \cap A_{k}$ e $t$ e $t^{\prime}$ sono le coordinate della proiezione di $\eta$ su $\Pi_{气}$ nei due diversi sistemi $\left[\xi, \tau_{1}, \ldots, \tau_{m}\right]_{h}$ e $\left[\xi, \tau_{1}^{\prime}, \ldots, \tau_{m}^{\prime}\right]_{k}$

(26) Cfr. [15] \& 23.

(27) Con $\bar{\delta}_{r s}$ indicheremo qui ed in seguito il simbolo di Kronecker; $\left\|\delta_{r s}\right\|_{n}$ indicheriz di conseguenza la matrice di ordine $n$ corrispondente alla trasformazione identica. 
$G_{h}(\xi, t)$ si ottenga da $G_{k}\left(\xi, t^{\prime}\right)$ eseguendo su $\tau_{1}^{\prime}, \ldots, \tau_{m-1}^{\prime}$ una sostituzione lineare ortogonale a determinare uguale a 1.

Supporremo inoltre che $a_{0}(\xi)$ e ciascuna $G_{h}(\xi, t)$ siano come funzioni di $\xi$ di classe $C^{(0, x)}$ uniformemente rispetto a $t \in \partial \boldsymbol{\Omega}_{m-1}$. Diremo simbolo della trasformazione $\mathfrak{d}$ l'insieme delle funzioni

$$
\sigma_{\mathfrak{E}, h}\left(\xi, t^{\prime}\right)=\alpha_{0}(\xi)+\frac{\mathfrak{F}}{t}\left(\frac{G_{h}(\xi, t, 0)}{|t|^{m-1}}\right)
$$

per $h=1, \ldots, N$.

Tenendo presente che per l'integrale a valor principale che figura nella (4.13) vale la formula $(3,12)$, si dimostrano gli analoghi dei teoremi (4.3) e (4.4). Il problema della riduzione, in questo caso, può essere affrontato come segue. Supposto che ciascuna delle $\sigma_{\mathfrak{G}, h}$ non sia mai nulla, è possibile costruire per ogni $\xi \in A_{k}$ due funzioni $b(\xi)$ e $\Psi_{k}(\xi, t)$ tali che risulti

$$
\left[\sigma_{\mathfrak{G},{ }_{k}}(\xi, t)\right]^{-1}=b_{0}(\xi)+\underset{t}{\mathfrak{F}}\left(\frac{\Psi_{k}(\xi, \tau)}{|\tau|^{m-1}}\right)
$$

proprio come abbiamo iudicato a proposito della riduzione nel caso in cui gli integrali sono estesi ad un $E_{m-1}$. Ln funzione $b_{0}(g)$ ha l'espressione

$$
\frac{1}{\operatorname{mis} \partial \Omega_{m-1}} \int_{\partial \Omega_{m-1}}\left[\sigma_{a_{n}}(\xi, t)\right]^{-1} d_{t} \sigma
$$

la quale, si constata facilmente, non dipende dall'indice $k$ per la menzio. nata proprietà della $G_{h}(\xi, t)$. Ciò posto, per ogni fissato $\xi \in A_{k}$, indichiamo con $3 d_{k}(\xi)$ l'estremo superiore dell insieme numerico descritto dagli $\varepsilon$ tali che $I(\xi, s) \subset A_{k}$; consideriamo quindi una funzione $\Pi_{k}(\xi, \eta)$ tale che

$$
\Pi_{k}(\xi, \eta)= \begin{cases}1 & \text { se } \eta_{i} \in I\left(\xi, d_{k}(\xi)\right) \\ 0 & \text { se } \eta \notin I\left(\xi, 2 d_{k}(\xi)\right)\end{cases}
$$

e risulti inoltre di classe $C^{(1)}$ in $A_{k} \times A_{k}$.

Fissato un punto $\eta \in I(\xi, d)$ e detta $\tau$ la proiezione di $\eta$ su $\Pi_{s}$, poniamo

$$
\Phi_{k}(\xi, \eta)= \begin{cases}\Psi_{k}(\xi, \tau) \Pi_{k}(\xi, \eta) & \text { per } \eta \in I\left(\xi, 3 d_{k}(\xi)\right) \\ 0 & \text { per } \eta \notin I\left(\xi, 3 d_{k}(\xi)\right) .\end{cases}
$$

亡̀ evidente che la funzione $\Phi_{k}(\xi, \eta)$ così definita è continua, fissato 
$\xi \in A_{k}$, in qualunque $\eta \in \partial T-\{\xi\}$. Indichiamo ora on $\mu_{1}, \ldots, \mu_{k}$ una par. tizione dell' unità subordinata al ricoprimento $A_{1}, \ldots, A_{N}$, e poniamo:

$$
\sum_{k=1}^{N} \mu_{k}(\xi) \Phi_{k}\left(\xi, \eta_{i}\right)=\Phi\left(\xi, \eta_{i}\right)
$$

Si riconosce che

$$
\mathfrak{A}_{1}(\varphi)=b_{0}(\xi) \varphi(\xi)+\int_{\partial T}^{*} \frac{\Phi(\xi, \eta)}{\xi^{\prime}-\eta^{m-1}} \varphi(\eta) d_{\eta} \sigma
$$

¿̀ tale ohe

$$
\mathfrak{A}_{1} \mathfrak{d}=I+\tau
$$

essendo

$$
\tau(\varphi)^{\prime}=\int_{\partial T} L(\xi, \eta) \varphi(\eta) d_{\nu, \sigma}
$$

con $L \in N^{(\beta)}(3 T)$ con $\beta>0$. Ragionando come per gli operatori della classe $\mathfrak{A}$ si trova che vi è una trasformazione riducente e si può perciò concludere col

Teorema (4.7) - Se ciascuna delle funzioni $\sigma_{\mathfrak{Q}, k}$ non è mai nulla $k=1, \ldots, N$, la trasformazione $\mathfrak{A} \dot{e}$ a codominio chiuso ed ha al piu un numero finito di autosoluzioni linearmente indipendenti $\left({ }^{28}\right)$.

5) Traduzione del problema $I$ in un sistema di equazioni integrali.- Riferendoci al sistema (1.1) poniamo

$$
\begin{aligned}
& a_{, s}[\lambda]=\sum_{p=1}^{m} \alpha_{r s}^{p} \lambda_{p} \\
& \sum_{=1 s}^{2 n} A_{r s}[\lambda] \alpha_{s t}[\lambda]=\delta_{r t} \quad \text { per } \lambda \neq 0:
\end{aligned}
$$

e per ogni $z \neq O$

$\left(\tilde{\jmath} .1^{\prime \prime}\right) \quad M_{r s}(z)= \begin{cases}\frac{(-1)^{m^{\prime}}}{4(2 \pi)^{m-1}} \Delta_{z}^{n^{\prime}} \int_{\partial \mathbf{Q}_{n z}} A_{r s}[\lambda] s g(z, \lambda) d_{\lambda} \sigma & \text { se } m=2 m^{\prime}+1 \\ \frac{(-1)^{m^{\prime}+1}}{(2 \pi)^{m}} \Delta_{z}^{m^{\prime}} \int_{\partial \mathbf{\Omega}_{m}} A_{r s}[\lambda](z \cdot \lambda) \log |\lambda \cdot z| d_{2, \sigma} & \text { se } m=2 m^{\prime} .\end{cases}$

(28) L'impostazione di quest'ultimo argomento è stggerita da GrRaud in [10] e da MrhulN in [15]. le conclusioni tratte però sono basate sui citati risultati di CaLderon e ZYGMIUND. 
Si dimostrano le seguenti proposizioni:

$$
M_{r} \text { è positivamente omogenea di grado } m-1 \text {. }
$$

$$
M_{, s} \text { è dispari, ciò̀: } M_{r s}(-z)=-M_{r s s}(z) \text { per ogni } z \neq 0 \text {. }
$$

(5.JV) | $M_{s \cdot s}(y-x) \|$ è una matrice fondamentale ${ }^{\left({ }^{29}\right)}$ del sistema (1.1).

Ci sarà comodo introdurre le funzioni

$$
C_{r s}(z)=M M_{r s}\left(\frac{z}{|z|}\right)
$$

per mezzo delle quali si avrà

$$
M_{r s}(y-x)=\frac{C_{r s}(y-x)}{|y-x|^{m-1}} .
$$

Si verifica immediatamente che le funzioni $C_{n_{s}}$ soddisfano alle ipotesi $\left.I_{4}\right)$ e $I_{5}$ ) del n. 3 .

Detta $\left\{u_{1}, \ldots, u_{2 n}\right\}$ una qualunque soluzione del sistema (1.1) di classe $\left.C^{(0)}(T) \cap C^{1}\right)(T-\partial T)$, si dimostra la seguente formula $\left({ }^{30}\right)$ :

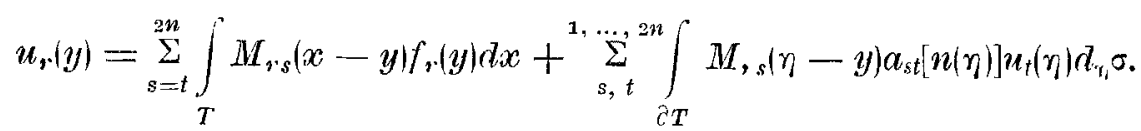

Per i potenziali

$$
w_{r s}(x)=\int_{\hat{o} T} M_{r \cdot s}(\eta-x) \varphi(\eta) d_{n} \sigma
$$

valgono i risultati stabiliti nel n. 3), e pertanto per i teoremi (3.5) e (3.8) esistono $(2 n)^{2}$ funzioni $m_{r s}$ di classe $C^{(0, x)}(\partial T)$ tali che per ogni $\varphi \in C^{(0, \alpha)}(\partial T)$ si abbia:

$$
w_{r s}^{ \pm}(\xi)= \pm \frac{1}{2} m_{r_{s}}(\xi) \varphi(\xi)+\int_{\partial T}^{*} M_{1 s}\left(\eta-\xi \mid \rho(\eta) d_{\eta} \sigma\right.
$$

(29) Cfr. per esempio [1t], Cap. III.

(30) Cfr. [14] e [13]. 
ci proponiamo di far vedere che si ha

$$
m_{r s}(\xi)=A_{r_{r}}[n(\xi)] \quad \text { per ogni } \xi \in \partial T .
$$

A tale scopo osseviamo che, fissato un intero $k$ tra 1 e $2 n$ e posto

$$
u_{r},(x)=\delta_{r k} \quad \text { per ogni } x \in T \text { e per } r=1, \ldots, 2 n,
$$

si ottiene una soluzione del sistema omogeneo associato al sistema (1,1) e pertanto la formula (5.2) ci dà

$$
\delta_{r k}=\sum_{s=1}^{2 n} \int_{\partial T} M_{r s}(\eta-x) a_{s k}[n(\eta)] d_{r_{i}} \sigma \quad \text { per } x \in T-\partial T ;
$$

da cui applicando la $(5.3)$ si ricava:

$$
\delta_{r k}=\frac{1}{2} \sum_{s=1}^{2 n} m_{r s}(\xi) a_{s k}[n(\xi)]+\sum_{s=1}^{2 n} \int_{\partial T}^{*} M_{r_{s}}(\eta-\xi) a_{s k}[n(\eta)] d_{\eta} \sigma .
$$

D'altra parte una semplice applicazione della formula di Gauss mostra che

$$
0=\sum_{s=1}^{2 n} \int_{\partial T} M_{r_{s}}\left(x_{i}-x\right) \alpha_{s k}\left[n(\eta) d_{r_{1}} \sigma \quad \text { per ogni } x \in \mathbb{e} T\right.
$$

e da questa si deduce

$$
0=-\frac{1}{2} \sum_{s=1}^{2 n} m_{r s}(\xi) \alpha_{s k}[n(\xi)]+\sum_{s=1}^{2 n} \int_{\partial T}^{*} M_{r s}(\eta-\xi) \alpha_{s k}[n(\eta)] d_{\eta} \sigma
$$

e quindi, sottraendo dalla (j.4) in definitiva:

$$
\sum_{s=1}^{2 n} m_{r s}(\xi) a_{s k}[n(\xi)]=\delta_{r k}
$$

Tenendo presente la $\left(5.1^{\prime}\right)$ e l'ipotesi di elliticità, si deduce la $\left(5.3^{\prime}\right)$.

Per trattare ora il problema I consideriamo con lo spazio $C^{(0, \alpha)}{ }_{\{} \Gamma_{\left.\}_{A}\right)}$ un altro spazio $C^{(0, x)}\left(\{\Theta\}_{B}\right)$ relativo ad un ricoprimento $\left\{B_{1}, \ldots, B_{M}\right\}$ di $\partial T$ e ad un sistema di matrici $\Theta^{(h, k)}$ che per il momento lasciamo indeterminate. È lecito fissare un numero positivo $d$ tale the per ogni punto $\xi \in \partial T$ esistono un insieme $A_{k} \in\{A\}_{N}$ e uno $B_{h} \in\{B\}_{M}$, tali che $I(\xi, d) \subset A_{k} \cap B_{h}$. Fissiamo quindi $M$ matrici ad $n$ righe e $2 n$ colonne $\left\|d_{l r}^{(k)}\right\| k=1, \ldots, M$, in modo che ciascuna delle $M-$ ple di vettori $d_{r}^{(k)}=\left(d_{1, r}^{(k)}, \ldots, d_{n, r}^{(k)}\right) k=1, \ldots, M$ ottenuta 
per ogni fissato $r$, sia un elemento di $C^{(0, \alpha)}\left(H_{B}\right)$. Detto allora $\varphi$ un elemento arbitrario di $C^{\langle 0 . \alpha\rangle}\left(\left\{\Theta^{* i}{ }_{B}\right\}\right.$ poniamo

$$
\begin{aligned}
u_{t}(x) & =\sum_{k=1}^{M} \sum_{r=1}^{2 n} \int_{D_{k}} M_{t r}(\eta-x)\left[\sum_{i=1}^{n} d_{i r}^{(k)}(\eta) \varphi \varphi^{(k)}(\eta)\right] d_{n} \sigma \\
& -\sum_{r=1}^{2 n} \int_{T} M_{t r}(y-x) f_{r}(y) d y
\end{aligned}
$$

avendo indicato con $D_{1}, \ldots, D_{M}$ una decomposizione opportunamente regolare di $\partial T$, per comodità tale che $D_{j} \subset B_{j}$. In virtù del lemma (2.2), ponendo

$$
\varphi_{r}(\xi)=\sum_{l=1}^{n n} d_{l r}^{(k)} \varphi_{l}^{\langle k\rangle}(\xi) \quad \text { per ogni } \xi \in B_{k} \text { e } k=1, \ldots, 2 n
$$

si ottengono $2 n$ fanzioni di classe $C^{(0, \alpha)}(\partial T)$; ne segue che le funzioni $(5.5)$, in virtù delle proprietà stabilite nel n. 3), sono di classe

$$
C^{(0, \alpha)}(T) \cap C^{(1)}(T-\partial T)
$$

e non dipendono dalla particolare decomposizione $D_{1}, \ldots, D_{M}$ di $\partial T$.

Imponiamo ora alle funzioni fornite dalle (5.5) di verificare le condizioni al contorno (1,3). Prendiamo un punto $\xi$ qualunque di $\partial T$ e supponiamo che $\xi \in A_{p^{\prime}} \cap B_{p}$ : imponendo le suddette condizioni nel punto $\bar{\xi}$, tenendo presenti le proprietà dei potenziali $v_{r s}$ le formule (5.3) e (5.3') si ottiene

$$
\sum_{l=1}^{n} k_{l l}^{\left\langle p^{\prime}, p\right\rangle}(\xi, \xi) \varphi_{l}^{(p)}(\xi)+\sum_{q=1}^{M} \sum_{l==1}^{m} \int_{D q}^{*} K_{l}^{\left(p^{\prime}, q\right)}(\xi, \eta) \varphi_{l}^{(q)}(\eta) d_{\eta_{l}} \sigma=g_{j}^{\left(p^{\prime}\right)}(\xi)
$$

per $p^{\prime}=1, \ldots, N ; p=1, \ldots, M ;$ avendo posto per $\xi \in A_{p^{\prime}}$ ed $\eta \in B_{q}$

$$
\begin{aligned}
& k_{j l}^{\left(\mu^{\prime}, q\right)}(\xi, \eta)=2_{2}^{1}{ }_{r, s}^{1, \ldots}{ }^{2 n} A_{r s}[n(\xi)] c_{j r}^{\left(p^{\prime}\right)}(\xi) d d_{l s}^{\left(\varphi^{\prime}\right.}(\eta) \\
& K_{j l}^{\left(p^{\prime}, \alpha\right)}(\xi, \eta)={ }_{r, s}^{1, \cdot \Sigma^{2 n}} M_{r \cdot s}(\eta-\xi) c_{j r}^{\left(p^{\prime}\right)}(\xi) d_{l s}^{(q)}(\eta) \quad \text { per } \xi \neq \eta \\
& g_{j}^{\left(p^{\prime}\right)}(\xi)=c_{j_{0}}^{\left(p^{\prime}\right)}\left(\xi_{r, s}\right)+{ }^{1, \ldots, 2 n}{ }^{\left(p^{\prime}\right\rangle}(\xi) \int_{T} M_{r_{s}}(y-\xi) f_{s}(y) d y
\end{aligned}
$$


Le (5.6) si possono considerare come un sistema di $n N$ equazioni integrali singolari nelie $n M$ incognite $\varphi_{1}^{(p)} \ldots \varphi_{n}^{(p)}$. È più comodo però associare al sistema $(5.6)$ una trasformazione da $\left.C^{(0, \alpha)}\left\{A^{*}\right\}_{B}\right)$ in $C^{(0, \alpha)}\left(\{\Gamma\}_{A}\right\}$ che scriveremo brevemente

$$
\widehat{\varphi}=\mathbb{S}(\varphi)
$$

essendo $\left.\bar{\varphi} \equiv\left(\widehat{\varphi}^{(1)}, \ldots, \bar{\varphi}^{(N)}\right)\right)$ e $\overline{\varphi^{\left(p^{\prime}\right)}} \equiv\left(\widehat{\varphi}_{1}^{(p)}, \ldots, \widehat{\varphi}_{n}^{\left(p^{\prime}\right)}\right)$ con

$$
\left.\widehat{\varphi}_{j}^{\left(p^{\prime}\right)}(\xi)=\sum_{l=1}^{n} k_{j, i}^{\left(p^{\prime}\right.}, p\right)\left(\xi, \xi \mid \varphi_{l}^{(p)}(\xi)+\sum_{q=1}^{M} \sum_{l=1}^{n} \int_{D_{q}}^{*} K_{j l}^{(p, q)}(\xi, \eta) \varphi_{l}^{(q)}(\eta) d_{\eta} \sigma\right.
$$

sì che il sistema (5.6) si scriverì anche sotto la forma

$$
S(\varphi)=g .
$$

In virtù del teorema (3.9) potremo considerare la trasformazione (5.6) da $L^{(2)}\left(\left\{\Theta^{*}{ }_{B}\right)\right.$ in $L^{(2)}\left(\left\{\boldsymbol{\Gamma}_{\mid A}\right)\right.$; ed in tali spazi ci proponiamo per ora di risol. vere l'equazione $\left(5.6^{\prime \prime}\right)$.

Indichiamo, conformemente alla convenzione adottata nel n. 2), con $C_{r, s: k}(\xi, t, 0)$ la restrizione di $C_{r, s}(y-\xi)$ al piano $\Pi_{\xi}$, essendo $\xi \in A_{k}$ e rife. rendoci al sistema di coordinate $\left[\xi, \tau_{1}, \ldots, \tau_{m-1}, \tau_{m}\right]_{k}$; poniamo quindi

$$
\begin{aligned}
& \tilde{C}_{r, s}\left(\xi, y^{\prime}-\xi\right)=\tilde{C}_{r, s ; k}\left(\xi, t^{\prime}\right)=\frac{2}{i} \underset{t}{\mathfrak{F}}\left[C_{r, s ; k}(\xi, t, 0) /|t|^{m-1}\right]
\end{aligned}
$$

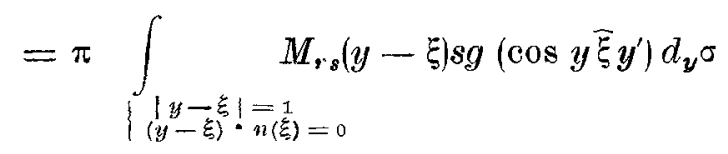

per $\left(y^{\prime}-\xi\right) \cdot n(\xi)=0$, essendo $l^{\prime}=\left(\tau_{1}^{\prime}, \ldots, \tau_{m-1}^{\prime}, 0\right)$ le componenti di $y^{\prime}-\xi$ nel sistema $\left[\xi, \tau_{1}, \ldots, \tau_{m}\right]_{k}$. La $(5.7)$ si ricava immediatamente dalla (4.3) osservando che

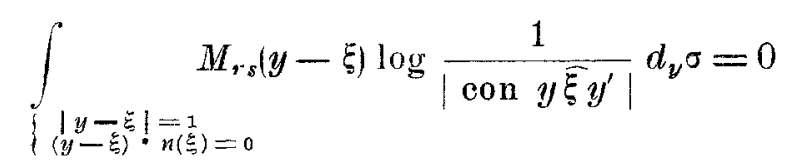

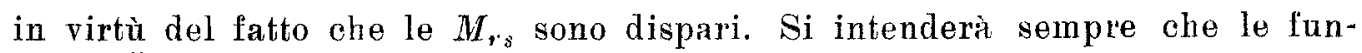
zioni $\tilde{C}_{r s}\left(\xi, y^{\prime}-\xi\right)$ sono definite per $\left|y^{\prime}-\xi\right|>0$ e $\left(y^{\prime}-\xi\right) \cdot n(\xi)=0$. 
Posto inoltre

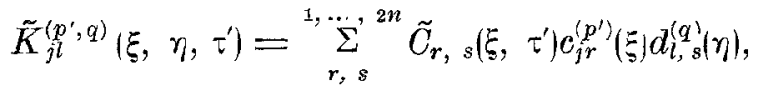

chiameremo matrici simboliche associate al sistema (5.6) o alla trasforma. zione $\left(5.6^{\prime}\right)$ le $N M$ matrici $\mathscr{T}\left(p^{\prime}, q\right)$ così definite

$$
\mathscr{9 K}\left(p^{\prime}, q\right)\left(\xi, \eta, \tau^{\prime}\right)=\left\|k_{j}^{\left(p^{\prime}, i\right.}{ }^{q)}(\xi, \eta)+i \tilde{K}_{j, i}^{\left(p^{\prime}\right.} i^{q)}\left(\xi, \eta, \tau^{\prime}\right)\right\|,
$$

per ogni $\xi \in A_{p}$, ed $\eta \in B_{q}$ e $\tau^{\prime} \in \Pi_{\xi} \cap \partial \mathrm{Q}_{m}(\xi, 1)$; porremo anche, per comodità

$$
\begin{gathered}
\mu_{j l}^{\langle(, q)}(\xi, \eta, \tau)=k_{j l}^{(p, q)}+i \tilde{K}_{j l}^{(p, q)} \\
=\frac{1}{2}{ }_{r, s}^{1, \ldots, 2 n}\left[A_{r s}[n(\xi)]+i \tilde{C}_{r, s}(\xi, \tau)\right] c_{j^{r}}^{(p)}(\xi) d_{l s}^{(q)}(\eta) .
\end{gathered}
$$

Per comprendere l'importanza di queste matrici osserviamo che, se $\{\Gamma\}_{A}$ coincide con $\left\{\Theta^{*}\right\}_{B}$ e la matrice $\mathscr{9 K}\left(p^{\prime}, p\right)(\xi, \xi, \tau)$ coincide in $A_{p^{\prime}} \cap A_{p}$ con la matrice $\left\|\delta_{, s}\right\|$ il nucleo $K_{j l}^{\left(p^{\prime} \cdot p\right)}$ per ogni $j, l=1, \ldots, n$ è di classe $N^{(\beta)}$ in questo insieme. Questa valutazione puó essere fatta applicando localmente i teoremi (4.3) e (4.4).

Dimostriamo ora il seguente

TeOrema $(5.1)$ - Fissato un qualunque punto $\xi \in \partial T$ e supposto $\xi \in A_{p} \cap B_{p}$, si abbia

$$
\operatorname{det} \mathfrak{O K}\left(p^{\prime}, p\right)(\xi, \xi, \tau) \neq 0
$$

\section{qualunque siano}

$$
\tau \in \Pi_{\varrho} \cap \partial \Omega_{m}(\xi, 1), p^{\prime} \in\{1, \ldots, N\} \text { e } p \in\{1, \ldots, M\}
$$

in queste ipotesi esiste una trasformazione $\mathfrak{S}^{\prime}$ definita in $L^{(-)}\left(\{\Gamma\}_{A}\right)$ ed avente codominio contenuto in $L^{(2)}\left(\left\{\theta^{*}{ }_{B}\right\}\right.$ tale che si abbia:

$$
\mathfrak{S}^{\prime} \mathfrak{S}=\mathfrak{J}+\tau
$$

essendo ờ identità e $\tau$ una trasformazione di $L^{(2)}\left\{\left(\Theta^{*} *_{B}\right)\right.$ in sè complelamente continua.

In base alla natura dei coefficienti $c_{r s}^{(p)}$ e $d_{r s}^{(q)}$ si stabilisce che, se il punto $\xi$ appartiene ad $A_{p^{\prime}} \cap A_{q^{\prime}}$ ed $\eta \in B_{p} \cap B_{q}$, si ha:

$$
\mathscr{T K}^{\left(p^{\prime}, p\right\rangle}=I^{\left(p^{\prime}, q^{\prime}\right)} \cdot \mathfrak{T K}^{\left(q^{\prime}, q\right)} \cdot \theta^{*(q p)} .
$$


Infatti si verifica facilmente che

$$
\mu_{j l}^{\left(p^{\prime}, p\right)}(\xi, \eta, \tau)=\sum_{h=1,}^{n} \sum_{k=1}^{n} \gamma_{j h}^{\left(p^{\prime}, q^{\prime}\right)}(\xi) \theta\left\langle(p, q)(\eta) \mu_{i k}^{\left(q^{\prime}, \alpha\right)}(\xi, \eta, \tau)\right.
$$

che significa proprio la (j.9). Dalla continuità degli elementi che compongono le suddette matrici e dalle (5.8) e (5.9) si deduce:

$$
\inf _{\tau \in \Pi_{\xi} \cap \partial \Omega_{M i}(\xi, 1), \xi \in A p^{\prime} \cap B p}\left|\operatorname{det} \mathscr{T K}\left(p^{\prime}, p\right)(\xi, \xi, \tau)\right|>0
$$

Fissato quindi un punto $\xi \in \partial T$ supposto che $l(\xi, d) \in A_{k} \cap B_{h}$ determiniamo un numero positivo $2 \sigma \leq d$ tale che si abbia

$$
\operatorname{det} \mathfrak{T K}^{(k, h)}(\xi, \eta, \tau) \neq 0 \quad \operatorname{det} \mathfrak{T K}^{(k, h)}(\eta, \xi, \tau) \neq 0
$$

per ogni $\eta \in I(\xi, 2 \sigma)$. Si può far vedere che è possibile fissare nn $\sigma$ valevole per ogni $\xi \in \partial T$ ed ogni $\tau$. A tale scopo indichiamo con $2 \bar{\sigma}(\xi, \tau)$ l'estremo superiore dell'insieme numerico descritto dai numeri $2 \sigma \leq d$ tali che per $\eta \in I(\xi, 2 \sigma)$ siano verificate entrambe le $\left(5.8^{\prime}\right)$. È facile ora vedere, sfruttando la compattezza di $\partial T$ e di $\partial \Omega_{m-1}$, che $\bar{\sigma}(\xi$, $v)$, al variare di $\xi$ su $\partial T$ e $\tau$ su $\Pi_{\xi} \cap \partial \Omega(\xi, 1)$, descrive un insieme numerico avente estremo inferiore positivo. Detto ancora $\sigma$ tale estremo inferiore, dalla (5.9) si deduce facilmente che, qualunque sia $\xi \in A_{p^{\prime}}$ (e per $\left.p^{\prime}=1, \ldots, N\right)$, si ha:

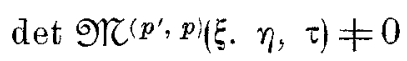

per ogni $\eta \in I(\xi, 2 \sigma) \cap B_{q}$ per ogni $q$ tale che $I(\xi, 2 \sigma) \cap B_{q}$ è non vuoto; analogamente per ogni $\xi \in B_{p}$ (e per $p=1, \ldots, M$ ) si ha:

$$
\operatorname{det} \mathfrak{T K}^{\left(p^{\prime}, q\right)}(\eta, \xi, \tau) \neq 0
$$

per ogni $\eta \in I(\xi, 2 \sigma) \cap A_{p^{\prime}}$ per ogni $p^{\prime}$ tale che $I(\xi, 2 \sigma) \cap A_{p^{\prime}} \dot{e}$ non vuoto. Indichiamo ora con $\mathscr{X}^{\left(p, p^{\prime}\right)}(\eta, \xi, \tau)$ la matrice inversa di $\left.\mathscr{N}\left(p^{\prime}, p\right) \xi \xi, \eta, \tau\right)$, quando $\eta \in I(\xi, 2 \sigma) \cap B_{p}$ e $\xi \in I(\eta, 2 \sigma) \cap A_{p^{\prime}} ;$ poniamo:

$$
\mathscr{\mathscr { T }}^{\left(p, p^{\prime}\right)}(\eta, \xi, \tau)=\left\|\gamma_{j l}^{\left(p, p^{\prime}\right)}(\eta, \xi, \tau)\right\|
$$

E' indispensabile osservare che sussistono le seguenti eguaglianze

$$
\begin{aligned}
& \sum_{l=1}^{n} \nu_{l j}^{(q, p)}(\eta, \xi, \tau) \mu_{i l}^{(p, q)}(\xi, \eta, \tau)=\delta_{j t} \\
& \sum_{l=1}^{n} \nu_{j l}^{(q, p)}(\eta, \xi, \tau) \mu_{i t}^{(p, q)}(\xi, \eta, \tau)=\delta_{l t}
\end{aligned}
$$


naturalmente per $\xi \in A_{p}$ ed $\eta \in B_{q} \cap I(\xi, 2 \sigma)$ oppure per $\eta \in B_{q}$ e $\xi \in A_{p} \cap I(\eta, 2 \sigma)$. Ora teniamo presente che dalla stessa definizione delle $\mu$ si ricava:

$$
\begin{array}{ll}
\mu_{t i}^{(p, q)}(\xi, \eta, \tau)=\sum_{h=1}^{n} \theta^{\left(q, q^{\prime}\right)}(\eta) \mu_{i h}^{\left(p, q^{\prime}\right)}(\bar{\xi}, \eta, \tau) & \gamma_{j} \in B_{q} \cap B_{q^{\prime}}, \\
\mu_{l t}^{(p, q)}(\xi, \eta, \tau)=\sum_{h=1}^{n} \gamma^{\left(p^{\prime} \cdot q\right)} \mu_{h t}^{\left(p^{\prime}, q\right)}(\xi, \gamma, \tau) & \xi \in A_{p} \cap A_{p^{\prime}}
\end{array}
$$

per cui sostituendo nelle $(5.11)$ e $\left(5.11^{\prime}\right)$ rispettivamente, e, tenendo presente la $\left(5.8^{\prime}\right)$. si deduce:

$$
\begin{aligned}
& v \frac{\left(q^{\prime}, p\right)}{h j}(\eta, \xi, \tau)=\sum_{l=1}^{n} \theta \frac{\left(q, q^{\prime}\right)}{l \hbar}(\eta) v^{(q, p)}(\eta, \xi, \tau) \\
& \nu_{l l}^{\left(q, p^{\prime}\right)}(\eta, \xi, \tau)=\sum_{l=1}^{n} \gamma^{\left(p, p^{\prime}\right)}(\xi) \nu_{l l}^{(q, p)}(\eta, \xi, \tau) .
\end{aligned}
$$

Le (5.13) e (5.13) significano che le matrici $\left\|\vee_{j l}^{(p, q)}\right\|$ per $\eta \in I(\xi, 2 \sigma)$ e $\tau \in \prod_{E} \cap \partial Q_{m}(\xi, 1)$ verificano le stesse condizioni ehe verificano gli elementi di $\left.C^{(0, \alpha)}\left(\mid \Theta^{*}\right\}_{B}, \Gamma \zeta_{A}\right)$, e cio esprimeremo dicendo che costituiscono un sistema di matrici localmente di classe $C^{(0, x)}\left\{\Theta^{*}\right\}_{B},\{\Gamma\}_{A} \mid$.

Ora osserviamo che, rispetto a $\tau$, ciascuna $\vee$ è positivamente omogenea di grado zero e di classe $C^{\infty}\left(E_{m-1}-\{O\}\right)$; fissati allora $\xi \in B_{p}$ ed $\eta \in A_{q}^{\prime}$, poniamo:

$$
h_{j l}^{(p, q)}(\xi, \eta)=-\frac{1}{\operatorname{mis} \partial \mathbf{Q}_{m-1}} \int_{\Pi_{\xi} \cap \partial \Omega_{n \ell}(\xi, 1)} \gamma_{j l}^{(p, q)}(\xi, \eta, \tau) d_{\tau} \sigma,
$$

e costruiamo la funzione $H_{j l}^{(p, q)}(\xi, \eta, t)$ tale che

$$
\underset{t}{\mathfrak{G}}\left(H_{j l}^{(p, q)}(\xi, \eta, t)\right)=-h_{j l}^{(p, q)}(\xi \cdot \eta)+v_{j l}^{(p, q)}(\xi, \eta, \tau),
$$

seguendo il procedimento suggerito dalla proposizione (4 IV).

Si riconosce $\left(^{31}\right)$ che le matrici $H_{j l}^{(p, q)}(\xi, \eta, t)$, che così si costruiscono, come le $\left\|h_{i_{1}}^{(p, q)}(\xi, \eta)\right\|$ e $\| v_{i_{j}}^{(p, q)}(\xi, \eta, z \|$, costituiscono un sistema di matrici localmente di classe $\left.C^{(0 . x)}\left(\left\{\Theta^{*}\right\}_{B}, \mid \Gamma\right\}_{A}\right)$.

(3i) Basta per questo applicare il teorema $(4,2)$. 
Poniamo allora

$H_{j l}^{(p, q)}(\xi, \eta)=\left\{\begin{array}{l}\Pi(\xi, \eta) H_{j}^{(p, q)}\left(\xi, \eta, t^{\prime}\right) \\ 0\end{array}\right.$

$$
\begin{aligned}
& \text { se } \eta \in I(\xi, 2 \sigma) \cap A_{q} \\
& \Rightarrow \eta \notin I(\xi, 2 \sigma)
\end{aligned}
$$

avendo indicato con $t^{\prime}$ la proiezione di $\eta$ su $\Pi_{\xi}$ e con $\Pi(\xi, \eta)$ una funzione di classe $C^{(1)}$ su $\partial T \times \partial T$, tale che

$$
\Pi(\xi, \eta)= \begin{cases}1 & \text { per } \eta \in I(\xi, \sigma) \\ 0 & \text { per } \eta \notin I(\xi, 2 \sigma)\end{cases}
$$

Ora osserviamo che per il modo come sono state definite le $H^{(p, q)}$, preso un qualunque elemento $\bar{\varphi} \in L^{(2)}\left(\{\Gamma\}_{A}\right)$, si può considerare

$$
\stackrel{\unrhd}{l=1}_{l=1}^{n} h_{l l}^{\left(p, p^{\prime}\right)}(\xi, \xi) \widehat{\varphi}_{l}^{\left(p^{\prime}\right)}(\xi)+\sum_{q=1}^{n} \sum_{l=1}^{n} \int_{D^{\prime} q}^{n} H_{j l}^{(p, q)}(\xi, \eta) \bar{\varphi}_{l}^{(q)}(\eta) d_{r^{\prime}} \sigma \quad \xi \in A_{p} \cap B_{p^{\prime}},
$$

che dà un elemento di $L^{(2)}\left(; \Theta^{*} !_{B}\right)$, il quale, in virtì della proposizione (2.2), non dipende dalla particolare decomposizione di $\partial T$. Indicando con $\mathcal{S}_{1}$ la trasformazione definita dalla (5.15), di si convince facilmente che per la trasformazione $\tau=\mathfrak{S}_{1} \mathfrak{S}-\mathfrak{g}$ esiste un intero $\vee$ tale che $\tau^{y}$ è completa. mente continua. Per questo basta fissare ad arbitrio una porzione opportunamente piccola di $\partial T$ ed applicare localmente il teorema (4.4). Ne segue allora che

$$
\left(\mathfrak{J}-\tau+\tau^{2}+\ldots+(-1)^{y-1} \tau^{y-\tau}\right) S_{1}=\mathfrak{S}^{\prime}
$$

è una trasformazione riducente la $\mathcal{S}$ e ciò dimostra completamente il nostro asserto.

Si comprende l'importanza del teorema (5.1) attraverso il

Teorema (5.2). - Nelle ipotesi del teorema (5.1), la trasformazione $\mathcal{S}$ defi. nita dalla (5.6') è a codominio chiuso ed ha un numero finito di autosoluzioni linearmente indipendenti.

Ciò è conseguenza immediata del teorema (2.5). In questo caso si vede che lo stesso risultato si estende al sistema aggiunto al sistema (5.6), e cio perchè, le matrici simboliche di quest'ultimo sono le trasposte coniugate di quelle corrispondenti del sistema (5.6).

Si ha perciò il

Teorema (5.3). - Nelle ipotesi del teorema (5.1), l'equazione omogenea associata alla $\left(5.6^{\prime \prime}\right)$ e la sna aggiunta hanno un numero finito di autosolu. 
zioni linearmente indipendenti. Condizione necessaria e sufficiente perchè l' equazione $\left(5.6^{\prime \prime}\right)$ sia risolubile $\dot{e}$ che il termine noto sia ortogonale ad ogni soluzione della omogenea trasposta associata.

Si deduce facilmente il

Teorema (5.4). - Se è verificata l'ipotesi del teorema (5.1) e l'equazione aggiunta omogenea associata alla $\left(5.6^{\prime \prime}\right)$ ha soltanto la soluzione banale, il problema I ammette almeno una soluzione.

6. Alcuni lemmi algebrici. - Data una forma quadratica non degenere

$$
f(u)={ }_{r, s}^{1, \ldots, m} a_{r s} u_{r} u_{s}
$$

poniamo

$$
\begin{aligned}
A & =\operatorname{det}\left\|a_{i k}\right\| \quad a^{r s}=\text { compl. alg. di } a_{r s} \text { in }\left\|a_{i k}\right\| \\
A_{r s} & =a^{r s} / A
\end{aligned}
$$

$$
F(U)=\sum_{r, s}^{1, \ldots} A_{r s} U_{r} U_{s}
$$

Sussistono le seguenti proposizioni

(6.I) Se $\rho_{1}, \ldots, \rho_{m}$ sono le radici dell' equazione secolare della forma (6.1), $\frac{1}{\rho_{1}}, \ldots, \frac{1}{\rho_{m}}$ sono le radici dell' equazione secolare della forma (6.2).

(6.II) Condizione necessaria e sufficiente perchè la sostituzione ortogonale

$$
u_{k}=\sum_{1}^{m} b_{k t} u_{t}^{\prime}
$$

trasformi la (6.1) nella sua forma canonica è che si abbia:

$$
\underset{r, s}{1, \ldots, m} a_{r s} b_{i r} b_{j s}= \begin{cases}0 & \text { per } i \neq j \\ \rho_{i} & \text { per } i=j\end{cases}
$$

avendo $\rho_{i}(i=1, \ldots, m)$ il significato che ha in $\left.\mathrm{I}\right)$.

(6.III) Se sono verificate le (6.3) sono verificate anche le condizioni

$$
\underset{r, s}{1, \ldots, m} A_{r s} b_{i r} b_{j s}= \begin{cases}0 & \text { per } i \neq j \\ \frac{1}{\rho_{i}} & \text { per } i=j\end{cases}
$$


e quindi la sostituzione

$$
U_{k}=\sum_{i}^{m i} b_{k t} U_{t}^{\prime}
$$

trasforma la (6.2) nella sua forma canonica.

Indichiamo ora con $p_{1}, \ldots . \rho_{\mu}$ le radici distinte dall' equazione secolare della $(6.1)$ e con $m_{1}, \ldots, m_{\mu}$ i relativi ordini di molteplicità ; si dimostra facilmente la proposizione

6.IV) Per avere una sostituzione orlogonale che trasformi la (6.1) nella sua forma canonica, basta considerare $i$ sistemi di equazioni lineari

$$
\sum_{r}^{n}\left(a_{r s}-\delta_{r s} \rho_{i}\right) X_{r}=0 \quad i=1, \ldots, \mu
$$

prendere $m_{1}$ soluzioni ortogonali e normali del sistema $\left(6,5_{1}\right)$ siano: $\left(b_{1,1}, \ldots\right.$ $\left.\ldots, b_{1, m}\right) . \ldots,\left(b_{m_{1}, 1}, \ldots . b_{m_{1}, m}\right) ;$ quindi $m_{2}$ soluzioni ortogonali e normali del si. stema $\left(6.5_{2}\right)$, siano $\left(b_{m_{1}+1,1}, \ldots, b_{n_{1}+1, m}\right), \ldots,\left(b_{m_{1}+m_{3}, 1}, \ldots, b_{m_{1}+m_{2}, m_{2}}\right) ; . . m_{\mu_{1}} s o-$ luzioni ortogonali e normali del sistema $\left(65_{l}\right)$, siano $\left(b_{m_{1}}+m_{2}+\ldots+m_{\mu-1}+1,1 \cdots\right.$ $\left.\ldots, b_{m_{1} \ldots+m_{\mu-1}+1, m}\right), \ldots,\left(b_{m, 1}, \ldots . b_{m, m}\right)$.

\section{OsservazTONE I}

La determinazione, per esempio, delle $\left(b_{1,1}, \ldots, b_{1, m}\right), \ldots,\left(b_{m_{1}, 1} \ldots, b_{m n_{1}, m}\right)$ è legata al minore di ordine $m-m_{1}$ diverso da zero, che si sceglie per risolvere il sistema $\left(6.5_{1}\right)$; ciò in generale potrà farsi in vari modi; se allora si prende una altra $m_{1}-$ pla di soluzioni di $\left(6.5_{1}\right)$, ottenuta fissando un minore di ordine $m-m_{1}$ diverso da quello che si è fissato per avere le precedenti, e sia $\left(b_{1,1}^{\prime}, \ldots, b_{1, m}^{\prime}\right), \ldots,\left(b_{m_{1}, 1}^{\prime}, \ldots, b_{m_{1}, m}^{\prime}\right)$, queste devono essere combinazioni lineari delle precedenti, esisterà perciò una matrice quadrata di ordine $m n_{1}$, diciamola $\|\underset{p q}{\theta(1)}\|$, tale che si abbia

$$
b^{\prime}{ }_{i k}=\sum_{1}^{m} \theta_{i h}^{(1)} b_{h k} \quad i=1, \ldots, m_{1},
$$

ed è facile riconoscere che tale matrice $\left\|\theta_{p q}\right\|$ è ortogonale in forza della ortogonalità delle $b$ e delle $b^{\prime}$. Infatti

$$
\begin{aligned}
\delta_{i j} & =\sum_{1}^{m_{1}}\left(\sum_{1}^{m_{1}} \theta_{i h}^{(1)} b_{h k}\right)\left(\sum_{I}^{m_{1}} \theta_{i t}^{(1)} b_{t k}\right) \\
& =\sum_{t, h}^{1 \ldots, \ldots, m_{1}} \sum_{1}^{m_{k}} \theta_{i h} \theta_{j t} b_{h h} b_{t h}=\sum_{t, h}^{1, \ldots, m_{l}} \theta_{i h} \theta_{j t} \delta_{h t}=\sum_{1}^{m_{1}} \theta_{i t} \theta_{j t} .
\end{aligned}
$$


Si dimostra facilmente il

Lemma $(6.1)$ - Se $T \grave{e}$ di classe $A^{(1, x)}$ e le $N$ funzioni $f_{1}, \ldots, f_{N}$ sono di classe $C^{(0)}$ su $a T$, non negative e non tutte nulle in uno stesso punto, qualunque esso sia, ̀̀ possibile decomporve $\partial T$ in un numero finito di regioni $D_{1}, \ldots, D_{S}$ chiuse su $\partial T$, in modo che a ciascuna regione $D_{i}$ si possa associare (almeno) una delle $f$ che risulti maggiore di zero su tulta $D_{i}$, e trovare un ricoprimento finito $\left\{B_{1}, \ldots, B_{s}\right\}$ di $\partial T$ meniante gli aperti $B_{i}$, in modo che a ciascuna $B_{i}$ si possa associare almeno una $f$ avente su $B_{i}$ estremo inferiore positivo.

Infatti per ipotesi la funzione

$$
f(\xi)=f_{1}(\xi)+\ldots+f_{N}(\xi)
$$

è continua e positiva su $\partial T$, avrà perciò un minimo assoluto positivo, indichiamolo con $\sigma$. Per l'ipotesi fatta su $\partial T$ e per la continuità delle $f_{i}$ è possibile decomporre $\partial T$ in un numero finito di regioni $D_{1}, \ldots, D_{s}$, chiuse su $\partial T$ in modo che in ognuna di esse l'oscillazione di ciascuna delle $f$ sia minore di $\frac{\sigma}{N}$.

È facile vedero che per ogni $D_{i}$, almeno una delle $f$ non si annulla in alcun punto di $D_{i}$, perchè, nel caso contrario, risulterebbe in $D_{\imath}$

$$
f_{1}(\xi)+\ldots+f_{N}(\xi)<\sigma
$$

e ciò è manifestamente assurdo. Segue di qui la prima parte del nostro asserto. La seconda è poi conseguenza immediata della continuità delle funzioni $f_{i}$.

Consideriamo la forma quadratica

$$
\sum_{r, s}^{1, \ldots, m} a_{r s}(\xi) u_{r} u_{s}
$$

supponendo le funzioni $a_{\text {ss }}$ di classe $C^{(0, x)}$ su $3 T$, ed indichiamo con $\left.p_{1}\left|\xi_{1}, \ldots, \rho_{y}\right| \xi_{\mid}\right)$le radici distinte dell' equazione

$$
\left|a_{r s}(\xi)-\delta_{r s} \rho\right|=0
$$

con $m_{1}, \ldots, m_{k}$ i loro rispettivi ordini di molteplicità.

Consideriamo la seguente ipotesi

$\left.\mathrm{I}_{6}\right)$ Ciascuna delle radici $\rho_{i}(\xi)$ dell equazione secolare associata alla forma quadratica (6.7) abbia ordine di molteplicità costante al variare di $\xi$ 
su $\partial T\left({ }^{32}\right)$. Tale ipotesi significa che ciascuna delle matrici

$$
\left|a_{r s}(\xi)-\delta_{r s} p_{i}(\xi)\right| \quad i=1, \ldots, \mu
$$

ha caratteristica costante al variare di $\xi$ su ogni regione connessa di $\partial T$ e uguale ad $m-m_{\imath}$.

Ci proponiamo di dimostrare il

Thorema ${ }^{2}(6.1)$ - Se $T$ è di classe $A^{(1, x)}$, le $a_{r s} d i$ classe $C^{(0, x)}$ su aT $e$ tali che sia verificata l' ipotesi $\left.\mathrm{I}_{6}\right)$ è possibile trovare un ricoprimento di $\partial T$, mediante un numero finito di aperti $B_{1}, \ldots, B_{S}$, e determinare in ogni regione $B_{k}\left(k=1, \ldots, S\right.$, una matrice ortogonale $\mathfrak{D}^{(k)}=\left\|d_{r s}^{(k)}\right\| d i$ ordine $m$ e con elementi tutti di classe $C^{(0, \alpha)}$ su $B_{k}$, tale che si abbia identicamente:

$$
\sum_{r, s}^{1, \ldots} a_{r s}^{m}(\xi) u_{r} u_{s}=\sum_{l=1}^{m} \rho_{l}(\xi)\left|\sum_{1}^{m} d_{l j}^{(\xi)}(\xi) u_{s}\right|^{2}
$$

per ogni $\xi \in B_{k}$, e per $k=1, \ldots, S$ (nella somma a secondo membro ogni radice $\rho_{l}$ è ripetuta $m_{l}$ volte).

Per dimostrare il teorema consideriamo la matrice

$$
\operatorname{OK}^{(l)}=\left\|a_{r s}(\xi)-\delta_{r s} P_{l}(\xi)\right\|
$$

ed indichiamo con $\sigma_{m-m_{l}}$ la somma dei quadrati di tutti i minori di ordine $m-m_{l}$ che si possono estrarre da $\mathscr{T}^{(l)}$; è evidente in virtù dell'ipotesi fatta sull'ordine di molteplicità di $\rho_{l}$, che $\sigma_{m-m_{l}}$ è maggiore di zero su tutta $\partial T$; ne segue in virtù del lemma (6.1), che è possibile effettuare una decomposizione di $\partial T$, diciamola $\Phi_{l}$, in un numero finito di regioni chiuse su $\partial T$, a ciascuna delle quali è possibile associare un minore di ordine $m-m_{l}$ di $\mathfrak{T K}^{(l)}$ che non si annulla in nessun punto della suddetta regione. Eseguendo allora le decomposizioni $\boldsymbol{\Phi}_{1}, \boldsymbol{D}_{2}, \ldots, \boldsymbol{D}_{\mu}$, aT resta divisa in un numero finito di regioni di $D_{1}, \ldots, D_{S}$ chiuse su $\partial T$, e tali che, comunque se ne prenda una, sia $D_{k}$, a questa si può associare, per ogni $l=1, \ldots, \mu$, un minore di ordine $m-m_{l}$ di $\mathfrak{K}^{(l)}$ che non si annulli in nessun punto di $D_{k}$. Avuti questi minori, il nostro asserto è immediata conseguenza della proposizione $(6.1 \mathrm{~V})$ e della continuità dei minori estratti. Rileveremo che, l'appartenenza dei coefficienti $d_{r s}^{\langle k\rangle}$ alla classe $C^{(0, \alpha)}$ su $D_{k}$, è conseguenza

(32) Abbiamo qui impostato le cose considerando $T$ ad unico contorno; gli accorgimenti da usare nel caso in cui $T$ ha un numero finito di contorni sono del tutto immediati. 
del fatto che $\mathrm{i}$ minori che si associano a $D_{k}$ sono funzioni continue anzi di classe $C^{(0, x)}$ e non nulle in $\bar{D}_{k}$. Per passare dalla decomposizione al ricoprimento di cui si parla nel teorema, basta sfruttare la continuità dei minori non nulli trovati su ciascuna $D_{k}$ e considerare un aperto $B_{k} \supset D_{k}$. su cui i suddetti minori sono ancora diversi da zero con i loro estremi. In virtù di quanto è stato detto a proposito della proposizione (6.IV) (osservazione I), per ogni coppia di regioni $B_{h} B_{k}$ aventi intersezione non vuota, resta definita una matrice ortogonale $\Theta^{(h, k)}$ di ordine $m$. tale che si abbia:

$$
d_{r s}^{(h)}=\sum_{1}^{\dot{m}_{t}} \theta_{r t}^{(h, k)} d_{l s}^{(k)} \quad \Theta^{(h, k)}=\left\|\theta_{r s}^{(h, k)}\right\|
$$

in ogni punto $\xi \in B_{h} \cap B_{k}$.

Se le matrici $\Theta^{(h, k)}$ fossero tutte coincidenti con la matrice identità, la riduzione della $(6,7)$ nella sua forma canonica sarebbe effettuata con coefficienti continui su tutta $a T$, eiò che evidentemente si può fare se le $a_{r s}$ sono costanti su $\partial T$.

OSSERVAZIONE II

La matrice $\Theta^{(h, k)}$ trovata è del tipo diagonale

$$
\left(\begin{array}{llll}
\Theta_{1}^{(h, k)} & 0 & & 0 \\
0 & \Theta_{2}^{(h, k)} & \ldots & 0 \\
\cdots & \cdots & \cdots & \cdots \\
0 & 0 & & \Theta_{\mu}^{(h, k)}
\end{array}\right)
$$

essendo ciascuna $\Theta_{i}^{(h, k)}$ matrice ortogonale di ordine $m_{i}(i=1, \ldots, \mu)$.

Consideriamo ora la forma quadratica

$$
\stackrel{1}{r s}^{2 n} a_{r s}[n(\xi)] u, u_{s}
$$

associata al sistema (1,1). verificante quindi l'ipotesi di ellitticità (1,2).

Dimostriamo il seguente

Teorema (6.2) - Nelle ipotesi poste l'indice di inerzia della forma quadratica

$$
F(\Lambda)={ }_{r, s}^{1, \ldots, 2 n}\left(\underset{1}{\sum_{p}} a_{r s}^{p} \lambda_{p}\right) \Lambda_{r} \Lambda_{s}
$$

$\grave{e}$ costantemente uguale ad $n$, qualunque sia $\lambda \in \mathrm{\partial Q}_{n}$. 
Per dimostrare il teorema, dobbiamo far vedere che l'equazione

$$
P(\lambda, \rho)=\operatorname{det}\left\|\sum_{1}^{m} a_{r s}^{p} \lambda_{p}-\tilde{o}_{r s p}\right\|=0
$$

ha $n$ radici positive ed $n$ negative per ogni $\lambda \in \partial \Omega_{m}$. Intanto le radici della (6.12) sono tutte reali in quanto il determinante è simmetrico; dalla ipotesi di ellitticità segue poi che sono tutte diverse da zero qualunque sia $\lambda$. Per ogni $\lambda \in \partial \Omega_{m}$ indichiamo con $\rho_{1}(\lambda)$ la più grande delle radici della (6.12). E ovvio che $\rho_{1}(\lambda)$ risulterà continua rispetto a $\lambda$ e mai nulla. D'altra parte, dal fatto che il determinante è di ordine pari, si ricava che $P(\lambda, \rho)=P(-\lambda,-\rho)$; per cui $P\left(\lambda, \rho_{1}(\lambda)\right)=0$ implica $P\left(-\lambda,-\rho_{1}(\lambda)\right)=0$. Poichè si passa con continuità da $-\lambda$ a $\lambda$ restando su $\partial \Omega_{m}$, si ricava che la (6.12) ha come radici $\rho_{1}(\lambda)>0, \rho^{\prime}(\lambda)=-\rho_{1}(-\lambda)<0$, qualunque sia $\lambda \in \partial \mathbf{Q}_{m}$.

Poniamo ora

$$
P_{1}(\lambda, \rho)=P(\lambda, \rho):\left\{\left(\rho-p_{1}(\lambda)\right) \cdot\left(\rho+\rho_{1}(-\lambda)\right)\right\} ;
$$

si ricava facilmente che

$$
P_{1}(\lambda, \rho)=P_{1}(-\lambda,-\rho)
$$

Si può perciò applicare all' equazione $P_{1}(\lambda, \rho)=0$ il procedimento dianzi descritto e giungere cosi all' esistenza di altre due radici $\rho_{2}(\lambda)$ e $-\rho_{2}(-\lambda)$. Così continuando si dimostra il. nostro asserto, $\theta$ anzi si stabilisce che se,

$$
\rho_{1}(\lambda) \geq \rho_{2}(\lambda) \geq \ldots \geq \rho_{n}(\lambda)>0
$$

sono le $n$ radici positive della (6.12) corrispondenti a $\lambda$, quelle negative saranno

$$
-p_{1}(-\lambda) \leq-p_{2}(-\lambda) \leq \ldots \leq-p_{n}\left(-\lambda_{1}\right)
$$

Per il teorema (6.1) applicato alla forma (6.10), possiamo conoludere eol

Teorema (6.3). - Se per la forma $(6.10)$ è verificata anche l'ipotesi $\left.\mathrm{I}_{6}\right)$, $\grave{e}$ possibile determinare un ricoprimento finito di $\partial T:\{B\}_{M}$ e su ogni $B_{c} \in_{\{B} \zeta_{M}$ una matrice ortogonale $\mathscr{D}^{(k)}=\left\|d_{\gamma^{\prime} s}^{(k)}\right\|$ di ordine $2 n$ con coefficienti di classe $C^{(0, \alpha)}\left(B_{k}\right)$ in modo che si abbia:

$$
\begin{gathered}
\sum_{r, s}^{1, \ldots, \sum^{2 n}} a_{r_{s}}[n(\xi)] u u_{r} u_{s}=\sum_{\rho=1}^{n} \rho_{l}(\xi)\left(\sum_{j=1}^{2 n} d_{l}^{(k)}(\xi) u_{l}\right)^{2} \\
+\sum_{l=1}^{2 n} \rho_{l}^{\prime}(\xi)\left(\sum_{j=1}^{2 n} d_{n+l, j}^{(k)}(\xi) u_{j}\right)^{2}
\end{gathered}
$$


per $\Xi \in B_{k}$ e qualunque siano le $\left(u_{1}, \ldots, u_{2 n}\right)$; dove $\rho_{1}(\xi), \ldots, \rho_{n}(\xi)$ sono le $n$ radici positive e $\rho^{\prime}(\xi) . \ldots, \rho_{n}^{\prime}(\xi)$ quelle negative dell' equazione secolare associatc alla forma (6.10) (ciascuna delle distinte è ripetuta tante volte quanto il sno ordine di molteplicita). Inoltre, per ogni regione non vuota del tipo $B_{h} \cap B_{k}$, esiste una matrice ortogonale $\left(A^{(h, k)}=\left\|\theta_{i}^{(h, k)}\right\|\right.$ con coefficienti $d i$ classe $C^{(0, \alpha)}$ tale che si abbia:

$$
d_{i t}^{(h)}=\sum_{l=1}^{2 n} \theta_{i l}^{(h, k)} d_{l i}^{(k)} \quad i=1, \ldots, 2 n
$$

per ogni $\xi \in B_{h} \cap B_{k}$

Liosservazione II ei permette di separare le condizioni (6.14) in due gruppi; quello in cui si fa variare $i$ da 1 ad $n$, e quello in cui $i$ varia da $n+1$ a $2 n$; tenendo presente che,

$$
\begin{aligned}
& \text { se } \quad 1 \leq i \leq n \quad \theta_{i l}^{(h, k)}=0 \quad \text { per ogni } l=n+1 . \ldots .2 n \\
& \text { se } \quad n+1 \leq i \leq n \quad \theta_{\imath l}^{(h . k)}=0 \quad, \quad, \quad l=1, \ldots, n,
\end{aligned}
$$

potremo scrivere le (6.14) nel modo seguente:

$$
\begin{aligned}
& d_{i t}^{(h)}(\xi)=\sum_{l=1}^{n} \theta_{i l}^{(h, k)} d_{l t^{\prime}}^{\langle k)} \quad i=1, \ldots, n \\
& d_{n+i, t}^{(h)}(\xi)=\sum_{l=1}^{n} \theta_{n+i, n+l}^{(h, k)} d_{n+l, t}^{(k)} .
\end{aligned}
$$

Noi continueremo a chiamare $\Theta^{(h, k)}$ la matrice di ordine $n$ che compare nelle $\left(6.14^{\prime}\right)$ e nelle $\left(6.14^{\prime \prime}\right)$ indifferentemente.

Le (6.14) possono scriversi brevemente

$$
\mathfrak{D}^{(h)}=\Theta^{(h, k)} \cdot \mathfrak{D}^{(k)}
$$

Da cui segue che se un punto appartiene a $B_{h} \cap B_{k} \cap B_{l}$, dovendosi avere

$$
\mathfrak{D}^{(k)}=\Theta^{(k, l)} \cdot \mathfrak{D}^{(l)}
$$

si deduce

$$
\mathfrak{D}^{(h)}=\left(\Theta^{(h, k)} \cdot \Theta^{(k, l)}\right) \cdot \mathfrak{D}^{(l)}
$$

e quindi in definitiva

$$
\Theta^{(h, l)}=\Theta^{(h, k)} \cdot(-)^{(k, l)}
$$


La formula $(6.15)$ vale sia per le matrici $\Theta$ di ordine $2 n$, sia per quelle di ordine $n$ che figurano nelle $\left(6.14^{\prime}\right)$ e $\left(6.14^{\prime}\right)$ separatamente. Resta cosi costruito un esempio di sistema di matrici relativo al ricoprimento $B_{1}, \ldots, B_{M}$ che verifica condizioni del tipo $(2.3)$.

7. Un teorema di esistenza ed unicità in un caso particolare. - Consideriamo i seguenti

Problema III. - Determinare una soluzione $\left\{u_{1}, \ldots, u_{2 n}\right\}$ di classe $C^{(0, a)}(\partial T) \cap C^{(1)}(T-\partial T)$ del sistema $(1,1)$, che, su ogni $B_{k}$ del ricoprimento $\{B\}_{M}$ di cui si parla nel teorema $(6.3)$, verifichi le condizioni

$$
\sum_{s=\tau}^{2 n} d_{r s}^{(k)}(\xi) u_{s}(\xi)=d_{r}^{(k)}(\xi)
$$

essendo $d_{r s}^{(k)}$ gli elementi delle prime $n$ righe della matrice $\mathfrak{D}^{(k)}$ di cui si parla nel teorema (6.3), e $d$ un elemento di $C^{(0, \alpha)}\left(\{\Theta\}_{B}\right)$.

Problema IV. - Determinare le funzioni $\left\{u_{1}, \ldots, u_{2 n}\right\}$ di classe

$$
C^{(0, \alpha)}(\mathfrak{e} T+\partial T) \cap C^{(1)}(e T)
$$

che verifichino in ogni punto di $\mathfrak{C} T$ il sistema omogeneo associato al sistema (1,1), ciascuna delle quali per $|x| \rightarrow \infty$ sia infinitesima di ordine superiore rispetto a $|x|-\frac{n}{2}$, le cui trace su $\partial(\mathcal{C} T)$ verifichino le stesse condizioni poste per il problema III.

Naturalmente, per poter considerare questi problemi è necessario ammettere l'esistenza delle funzioni $d_{r s}^{(k)}$ di cui si parla nel teorema (6.3), per questo è sufficiente che sia verificata l'ipotesi $\Upsilon_{6}$. Dimostriamo il

Teorema (7.1) - I problemi III e IV ammettono, al più, una soluzione.

Basta far vedere che è identicamente nulla ogni eventuale soluzione del relativo problema omogeneo associato. Se $\left\{u_{1}^{0}, \ldots, u_{2 n}^{0}\right\}$ è una soluzione del problema omogeneo associato al problema III [IV], verificante quindi le condizioni

$$
\begin{aligned}
\sum_{s=1}^{2 n} \sum_{p=1}^{m} \alpha_{r s}^{p} \frac{\partial u_{s}^{0}}{\partial x_{p}}=0 & \text { in } T-\partial T[\mathfrak{C} T] \\
\sum_{s=1}^{2 n k} d_{r s}^{(k)}(\xi) u_{s}(\xi)=0 & \text { in } B_{k}, \text { per } k=1, \ldots, M,
\end{aligned}
$$


per il teorema di Gauss, si avrà in entrambi i casi

$$
\int_{\partial T}^{1,} \sum_{r, s}{ }^{2 n} \alpha_{r s}[n(\xi)] u^{0}{ }_{r}(\xi) u_{s}^{0}(\xi) d_{\xi} \sigma=0 .
$$

D'altra parte, tenendo conto della formula (6.13) e della (7.2'), si ha:

$$
\sum_{k=1}^{M} \int_{D_{k}} \sum_{l=1}^{n} p^{\prime}(\xi)\left\{\sum_{j=1}^{2 n} d_{n+l, j}^{(h)}(\xi) u_{j}^{0}(\xi)\right\}^{2} d_{\xi} \sigma=0
$$

essendo $D_{1}, \ldots, D_{M}$ una arbitraria decomposizione di $\partial T$, tale che $D_{k} \subset B_{k}$; da quest'ultima eguaglianza, tenendo presente che tutte le pi hanno lo stesso segno, si deduce

$$
\sum_{j=1}^{2 n} d_{n+l, j}^{\langle k\rangle}(\xi) u_{j}^{0}(\xi)=0 \quad \text { per } l=1, \ldots, n
$$

in ogni $\xi \in B_{k}$. e ciò per $k=1, \ldots, M$. Dalle $\left(7.2^{\prime}\right)$ e $\left(7.2^{\prime \prime}\right)$ si deduce facilmente che le $u_{i}^{0}$ sono nulle su $\partial T$. Tenendo ora presente la formula

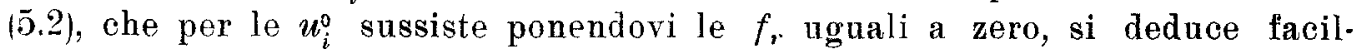
mente che in entrambi $\mathrm{i}$ casi le $u_{i}^{0}$ sono tutte identicamente nulle.

Per affrontare ora la questione esistenziale, relativa al problema III, usiamo la rappresentazione integrale (5.5). Osserviamo però che in questo caso gli spazi $C^{(0, x)}\left(\{\Theta\}_{B}\right)$ e $C^{(0, \alpha)}\left(\left\{(-)^{*}, B_{B}\right.\right.$ coincidono, per il fatto che le matrici $\Theta^{(h, k)}$ sono ortogonali.

Imponendo alle funzioni (5.5) di soddisfare alle condizioni al contorno (7.1) si trova un sistema di equazioni integrali a valor principale che si ottiene dal sistema (5.6) sostituendo in questo alle $c_{p r}^{(k)}$ le $d_{j r}^{(k)}$. Tenendo presente come sono state ricavate $l e d_{j r}^{(k)}$, si deduce che esse devono verificare le condizioni (6.4); pertanto, il sistema di equazioni integrali a valor prin. cipale cercato sarà

$$
\frac{1}{\rho_{i}(\xi)} \varphi_{i}^{(k)}(\xi)+\sum_{h=1}^{M} \sum_{l=1}^{n} \int_{D_{h}}^{*}{ }_{r, s}^{1,} \dddot{\Sigma}^{2 n} M_{r_{s}}(\eta-\xi) d_{i r}^{(k)}(\xi) d_{l s}^{(h)}(\eta) \varphi_{l}^{(h)}(\eta) d_{n} \sigma=g_{i}^{(k)}(\xi)
$$

per $i=1, \ldots, n$; per ogni $\xi \in B_{k}$, e per $k=1, \ldots, M$. Il sistema aggiunto omogeneo associato al (7.3) può essere ricavato facilmente tenendo presente il teorema (2.4); si trova:

$$
\frac{1}{\rho(\xi)} \psi_{l}^{(h)}(\xi)-\sum_{k=1}^{M} \sum_{i=1}^{n} \int_{D_{k}}^{*}{ }_{r, s}^{1, \ldots,{ }^{2 n}} M_{r s}(\eta-\xi) d_{i r}^{(k)}(\eta) d_{l s}^{(h)}(\xi) \psi_{i}^{(k)}(\eta) d_{n} \sigma=0
$$


per $l=1, \ldots, n$; per ogni $\xi \in B_{h}$ e per $h=1, \ldots . M$. Ora si constata facilmente che le equazioni (7.3') traducono le seguenti condizioni al contorno:

$$
\sum_{s=1}^{2 n} d_{l s}^{(h)}(\xi) v_{s}(\xi)=0 \quad \text { per ogni } \xi \in B_{h}
$$

per le funzioni

$$
v, r(x)=\sum_{k=1}^{M} \int_{D_{k}} \sum_{r=1}^{2 n} M_{r s}(\eta-x) \sum_{i=1}^{i} d_{i r}^{(k)}(\eta) \psi_{i}^{(k)}(\eta) d_{n} \sigma \quad r=1, \ldots, \bullet_{n}
$$

che sono per $|x| \rightarrow \infty$ infinitesime di ordine $m-1$ e verificano in ogni punto di $e T$ il sistema omogeneo associato al sistema (1.1) In virtù del teorema (7.1) si deduce che le $v,(x)$ corrispondenti ad una soluzione $\psi$ del sistema (7.3') sono identicamente nulle in $\mathfrak{C} T$. Affrontiamo ora lo studio del sistema (7.3) prendendo $\varphi=\left(\left(\varphi^{(1)}, \ldots . \varphi^{(N)} \|\right)\right.$ nello spazio $L^{(2)}\left(\{\Theta\}_{B}\right)$; in tale spazio andrà presa pertanto anche $\psi$ per una osservazione già fatta. Ragionando come abbiamo fatto per dimostrare il teorema (7.1), segue che le $v_{r}(x)$ corrispondenti ad una soluzione $\psi \in L^{(2)}\left(\{\Theta\}_{B}\right)$ del sistema $\left(7.3^{\prime}\right)$ sono identicamente nulle in $\mathcal{C}$ T. In virtì del teorema (3.9). per quasi tutti i punti $\xi \in B_{h}$ si avrè :

$$
\begin{aligned}
v_{r}^{\bar{*}}(\xi) & = \pm \frac{1}{2} \sum_{i=1}^{n} \sum_{s=1}^{2 n} A_{s r}[n(\xi)] d_{i r}^{(h)}(\xi) \psi_{i}^{(h)}(\xi) \\
& +\sum_{k=1}^{M} \int_{D_{k}}^{*} \sum_{s=1}^{2 n} M_{s r}(\eta-\xi) d_{i s}^{(k)}(\eta) \psi_{i}^{(k)}(\eta) d_{\eta} \sigma .
\end{aligned}
$$

Si deduce quindi che le $v_{r}(x)$ hanno le tracce interne date quasi ovunque dalle seguenti formule:

$$
v_{r}^{+}(\xi)=\sum_{i=1}^{n} \sum_{s=1}^{2 n} A_{r s}[n(\xi)] d_{i s}^{(h)}\left(\xi \mid \psi_{i}^{(h)}(\xi) \quad \text { per } \xi \in B_{h}\right.
$$

Poichè, come abbiamo già osservato in precedenza,

$$
\int_{\partial T}^{1, \ldots \Sigma_{r, s}^{2 n}} a_{r s}[n(\xi)] v_{r}^{+}(\xi) v_{s}^{+}(\xi) d \xi \sigma=0
$$

tenendo presenti le (6.4), si ha:

$$
\sum_{k=1}^{n} \int_{D_{k}} \sum_{j=1}^{n} \frac{1}{\rho_{j}(\xi)}\left|\varphi_{j}^{(k)}(\xi)\right|^{2} d_{\xi} \sigma=0
$$


da cui segue $\psi=0$. In maniera perfettamente analoga si può ragionare per una soluzione $\varphi$ del sistema omogeneo associato al sistema (7.3) Si può perciò concludere col seguente

Teorema (7.2) - Il sistema omogeneo associato al sistema 17.31 ed il suo aggiunto ammettono soltanto la soluzione banale.

Come abbiamo fatto nel n. $\bar{\jmath}$ per il sistema (5.6), consideriamo ora la trasformazione $\bar{\varphi}=\mathfrak{S}(\varphi)$, ottenuta dal sistema (7.3) come la $\left(\overline{5} .6^{\prime}\right)$ dal sistema (5.6). Ci proponiamo di dimostrare il

TeORema (7.3) - Nelle ipotesi ammesse, per la trasformazione $\mathcal{S}$ vale la tesi del teorema (5.1).

Per provare il nostro asserto basta far vedere che le matrici simboliche associate al sistema (7.3). che in questo caso si scriveranno $\mathscr{V}^{(k \cdot k)}(\xi ; \xi, \tau)$, perchè è stato adoperato un unico ricoprimento, sono non degeneri qualunque sia $\xi \in B_{k}$ e $\tau \in \Pi_{\xi} \cap \partial \Omega_{m}(\xi, 1)$. Posto

$$
\varphi_{j l}^{(k, k)}(\xi, \tau)={ }_{r, s}^{1, \ldots, 2 n} \tilde{C}_{r, s}(\xi, \tau) d_{j r}^{(k)}(\xi) d_{l s}^{(k)}(\xi) \quad \xi \in B_{k},
$$

dove le $\check{C}_{r s}$ sono definite dalle $(5.8)$, si ha:

$$
\mathfrak{M R}^{(k, k)}(\xi, \xi, \tau)=\left\|\frac{\delta_{j l}}{2 \rho_{j}}+i{ }_{2}^{\pi} \varphi_{j l}^{(k, k)}(\xi ; \tau)\right\|
$$

essendo le $\varphi_{j l}$ reali e simmetriche, si deduce facilmente

$$
\operatorname{det} \operatorname{TK}^{(k, k)}(\xi, \xi, \tau) \neq 0
$$

per ogni $\xi \in B_{k}$, qualunque sia $\tau \in \Pi_{\xi} \cap \partial \Omega_{m}(\xi, 1)$ e $k \equiv 1, \ldots, M$.

Infatti per $\xi$ e $\tau$ fissati, sia per assurdo:

$$
\left.\operatorname{det} \mathscr{T}^{(k, k)} \mid \xi, \xi, \tau\right)=0 \text {; }
$$

allora il sistema

$$
\sum_{l=\mathbf{1}}^{n}\left(\frac{\delta_{j l}}{\rho_{j}}+i \pi \varphi_{j l}^{(k, k)}\right) c_{l}=0
$$

uelle incognite $c_{l}$ ammette una soluzione diversa da quella banale, epperò dovrà risultare;

$$
\sum_{j, l}^{1, \ldots i}{ }^{2 n}\left(\frac{\delta_{j l}}{\rho_{j}}+i \pi \varphi_{j l}^{(k, k}\right) c_{l} \bar{c}_{j}=0
$$


da cui si deduce

$$
\sum_{j=1}^{n} \frac{\left|c_{i}\right|^{2}}{P_{j}}+i \pi \sum_{j, l}^{1, \ldots,{ }^{12}} \varphi_{j l}^{(k, k)} c_{l} \bar{c}_{j}=0
$$

ma questa eguaglianza implica

$$
\sum_{j=1}^{n} \frac{\left|c_{j}\right|^{2}}{\rho_{j}}=0 ; \sum_{j, l}^{1, \ldots, n}{ }_{\varphi_{1}^{(k, k)}}^{\left(k, c_{l}\right.} \bar{c}_{j}=0
$$

giacchè $\varphi_{j l}=\varphi_{l_{j}}$, come si ricava dalla ipotesi di simmetria da noi fatta sui coefficienti del sistema $(1,1)$; d'altra parte, poichè le radici $\rho_{j}$ hanno tutte lo stesso segno, si deduce $c_{j}=0$ per $j=1, \ldots, n$, contrariamente a quello che avevamo ammesso. Ciò dimostra la (7.6) nel senso che avevamo pre. cisato. Poichè le trasformazioni riducibili in uno spazio di BanacH sono a codominio chiuso, tenendo conto del teorema (7.2), si ha il

Teorema (7.4) - Il problema III ammette una ed una sola soluzione, qualunque siano $i$ termini noti.

Infatti per il teorema (7.3), la trasformazione (7.4) è a codominio chiuso; si deduce di qui, per il teorema (7.2), che il sistema (7.3) ammette una ed una sola soluzione $\varphi$ in $L^{(2)}\left(\{\Theta\}_{B}\right)$ qualunque sia il termine noto in $L^{(2)}\left(\{\Theta\}_{B}\right)$. Poichè le $g_{i}^{(k)}$ verificano una condizione di HöLDER, tenendo presente un recente teorema di MIHLIN, si deduce che ogni eventuale solnzione $\varphi$ del sistema (7.3) appartiene allo spazio $C^{(0, \alpha)}\left(\{\Theta\}_{B}\right)\left({ }^{33}\right)$. Ciò assicura che le funzioni $u_{t}$, costruite con le formule (5.5) e corrispondenti alla soluzione del sistema (7.3) sono di classe $C^{(0, \alpha)}(T)$, in virtì del teorema (3.2), e costituiscono quindi una soluzione del sistema (1.1), che soddisfa, a tutte le condizioni richieste nel problema $\mathrm{I}$.

Se il termine noto a secondo membro delle condizioni (7.1) appartiene soltanto ad $L^{(2)}\left(\{\Theta\}_{B}\right)$, si avrà un teorema di esistenza ed unicità per il problema che richiede di determinare le $\left\{u_{1}, \ldots, u_{2 n}\right\}$ nella classe delle funzioni che appartengono a $L^{(2)}(T) \cap C^{(1)}(T-\partial T)$ e verificano le (7.1) quasi ovunque ed il sistema (1.1) in tutti i punti di $T-\partial T$.

Da quanto abbiamo sopra stabilito si deduce il

TEonema (7.5) - Le formule (5.5) pongono unce corrispondenza biunivoca tra l'insieme delle soluzioni $\left\{u_{1}, \ldots, u_{2 n}\right\}$ del sistema (1.1) di classe $C^{(0, \alpha)}(T) \cap C^{(1)}(T-\partial T)$ e l' insieme $\left.C^{(0, x)}(\{\theta\}\}_{B}\right)$.

(3) Cfr. [18]; i risultati ivi stabiliti si riferiscono alle equazioni integrali in cui gli integrali a valor principale sono estesi all intero spazio o ad una rarietà; ma si riconosce agevolmente che quella trattazione si estende al nostro caso senza alcuna difficultì. 
Basta per questo osservare che, comunque si prenda un elemento del primo insieme $\left\{u_{1}^{*}, \ldots, u_{2 n}^{*}\right\}$ esiste uno ed un solo elemento $\varphi \in C^{(0, x)}\left(\{\Theta\}_{B}\right)$, tale che le $u$ corrispondenti a questo $\varphi$ mediante le (5.5) verifichino la condizione

$$
\sum_{s=1}^{2 n} d_{r s}^{(k)} u_{s}=\sum_{s=1}^{2 n} d_{r s}^{(k)} u_{s}^{*}
$$

Per il teorema di unicità, si deduce poi che $u_{s}=u_{s}^{*}$, mentre il teorema (7.2) assicura che questo $\varphi$ è unico. Ciò, con altre ovvie argomentazioni, prova il nostro asserto.

Notiamo esplicitamente che il teorema (7.5) continua a sussistere se si sostituisce al sistema (1.1) il suo omogeneo associato, tenendo conto naturalmente, ove occorra, che bisogna porre lo zero al posto delle $f_{r}$ ovunque queste figurino.

Ci pare di grande interesse considerare il seguente caso. Supponiamo $T$ limitato e a due contorni $S_{1}$ e $S_{2}$, siano $\left\{B^{\prime}\right\}_{M_{1}}$ e $\left\{B^{\prime \prime}\right\}_{M_{0}}$ i ricoprimenti di $S_{1}$ ed $S_{2}$ di cui si parla nel teorema (6.3). Poniamo allora il

Problema V. - Determinare una soluzione $\left\{u_{1}, \ldots, u_{2 n}\right\}$ di classe $C^{(0 . \alpha)}(T) \cap C^{(1)}(T-\partial T)$ del sistema (1.1) che verifichi le condizioni al contorno

$$
\begin{array}{ll}
\sum_{s=1}^{2 n} d_{r s}^{\prime(k)}(\xi) u_{s}(\xi)=d_{r}^{\prime(k)}(\xi) & \text { su }{B^{\prime}}_{k}^{(1)}, k=1, \ldots, M_{1} \\
\sum_{s=1}^{2 n} d_{n-r, s}^{\prime \prime(k)}(\xi) u_{s}(\xi)=d^{\prime \prime}{ }_{r}^{\prime(h)}(\xi) & \text { su }{B^{\prime \prime \prime}}_{h}^{\prime(2)}, h=1, \ldots, M_{2}
\end{array}
$$

avendo le $d_{r » s}^{\prime(k)}$ e le $d_{n+r, s}^{\prime \prime(h)}$ il significato che hanno nel teorema (3.6).

Si vede subito che per un tale problema la dimostrazione che noi abbiamo fatto del teorema (7.2) non può essere adattata, per il fatto che sul contorno $S_{1}$ si sono prese le $d_{r s}^{(k)}$ corrispondenti alle radici positive, e su $S_{2}$ le $d_{r s}^{(h)}$ corrispondenti alle radici negative dell' equazione secolare asso. ciata alla forma quadratica (6.10). È facile però vedere che seguita a valere il teorema (7.3). Per una classe di sistemi che abbiamo riportato nel n. 9, si riconosee che per un tale problema vale il teorema dell'alternativa.

8. - Teoremi di esistenza ed unicità nel caso generale. - Affrontiamo ora lo studio del problema I usando la rappresentazione integrale (5.5), supponendo che le funzioni $d_{r s}^{(k)}$ che ivi figurano siano proprio quelle di cui ci siamo serviti per formulare il problema III. Quanto segue, perciò, si basa sulla ipotesi $\left.I_{6}\right)$, che ha permesso di costruire queste funzioni $d_{r 3}^{(k)}$ 
(teorema (6.4)). Tenendo presente il teorema (7.5) e ripetendo lo stesso ragiunamento che ha portato al sistema (5.6), si può enunciare il

Teorema (8.1) - Se per la forma quadratica $(6.10)$ è verificala l' ipotesi $\left.\mathrm{I}_{6}\right)$, il problema I è equivalente alla equazione $\left(5.6^{\prime \prime}\right)$.

E bene precisare che tale equivalenza va intesa nel senso che se e solo se è risolubile il sistema o l' equazione $\left(5.6^{\prime \prime}\right)$ lo è il problema I e viceversa; inoltre, se esistono $p$ soluzioni linearmente indipendenti del sistema omogeneo associato all' equazione $\left(5.6^{\prime \prime}\right)$, esistono altrettante soluzioni linearmente indipendenti in $T$ del problema omogeneo associato al problema I. La dimostrazione è evidente.

Ci proponiamo ora di definire l'aggiunto omogeneo associato al problema I. A tale scopo scriviamo il sistema trasposto omogeneo associato al sistema (5.6), supponendo che $\varphi$ vari in $L^{(2)}\left(\{\Theta\}_{B}\right\}$. Da quanto abbiamo detto sulla forma del più generale funzionale in $L^{(2)}\left(\{\boldsymbol{\Gamma}\}_{A}\right)$ (teorema (2.4)), si ricava che il sistema cercato è il seguente

$$
\begin{aligned}
& \sum_{l=1}^{n} \sum_{r, s}^{1, \ldots, 2 n} A_{r s}[n(\xi)] c_{l r}^{(k)}(\xi) d_{j s}^{(k)}(\xi) \biguplus_{l}^{(k)}(\xi) \\
+ & \sum_{h=1}^{n} \sum_{l=1}^{n} \int_{D_{h}}^{*}{ }_{r, s}^{1, \ldots,{ }^{2 n}} M_{r s}(\eta-\xi) c_{r l}^{(h)}(\eta) d_{j s}^{(k)}(\xi) \psi_{l}^{(h)}(\eta) d_{n} \sigma=0
\end{aligned}
$$

essendo $\psi$ un elemento da ricercarsi in $L^{(2)}\left(\left\{\Gamma^{*}\right\}_{A}\right)$. Questo sistema di equazioni, in virtù del teorema (39), puo essere interpretato come il seguente sistema di condizioni al contorno

$$
\sum_{s=1}^{2 n} d_{j s}^{(k)}(\xi) v_{s}^{-}(\xi)=0 \quad j=1, \ldots, n
$$

per ogni $\xi \in B_{k}$, per $k=1, \ldots, M$, imposte alle funzioni

$$
v_{s}(x)=\sum_{h=1}^{N} \int_{D_{h}} \sum_{r=1}^{2 n} M_{r s}(\eta-x) \sum_{l=1}^{n} c_{l r}^{(h)}(\eta) \varphi_{l}^{(h)}(\eta) d_{\eta} \sigma .
$$

Siccome le funzioni $v_{1}(x), \ldots, v_{2 n}(x)$ fornite dalle (8.2) costituiscono una soluzione in eT del sistema omogeneo associato al sistema (1.1) e sono infinitesime come $|x|^{1-m}$ per $|x| \rightarrow \infty$, si ricava che, se $\psi$ è un' autosoluzione del sistema (8.1), le $v_{s}$ corrispondenti mediante le (8.2), in virtù del teorema (7.1), sono identicamente nulle in $\mathcal{C}$. Ragionando come abbiamo 
fatto a proposito del sistema (7.3), si trova

$$
v_{s}^{+}(\xi)=-\sum_{l=1}^{n} \sum_{r=1}^{2 n} A_{r s}[n(\xi)] c_{l r}^{(h)}(\xi) \biguplus_{l}^{(k)}(\xi)
$$

per ogni $\xi \in A_{k}$, e per $k=1, \ldots, N$. Le $(\varepsilon .3)$ suggeriscono di definire come aggiunto (omogeneo) del problema $I$ il seguente

Problema VI. - Determinare $2 n$ funzioni $\left\{v_{1}, \ldots, v_{2 n}\right\}$ di classe $C^{(0, x)}(T) \cap C^{(1)}(T-\partial T)$, che costituiscano una soluzione in $T-\partial T$ del sistema omogeneo associato al sistema (1.1), ed un elemento

$$
\psi=\left(\left(\psi^{(1)}, \ldots, \psi(N)\right)\right) \in C^{(0, a)}\left(\left\{T^{* *}\right\}_{A}\right)
$$

in modo che su $\partial T$ siano verificate le $(8.3)$, essendo le $c_{r s}^{(k)}$ le stesse che figurano nelle condizioni al contorno (1.3).

Indicheremo con $[v, \psi]$ una generica soluzione del problema VI; da quanto si è detto precedentemente discendons $\mathrm{i}$ seguenti

TeOrema (8.2) - La corrispondenza slabilita dalle formule (8.2) tra l' insieme delle autosoluzioni del sistema $\{8.1\}$ di classe $C^{(0, x)}\left(\left\{\Gamma^{*}\right\}_{A}\right\}$ e l'insieme delle soluzioni del problenuc VI è biunivoca dell' uno sull' altro.

TeOrema (8.3) - Condizione necessaria perchè il problema I sia risolubile è che per ogni soluzione $[v, \psi]$ del problema VI si abbia

$$
\sum_{k=1}^{N} \int_{D_{k}}\left(\sum_{j=1}^{n} g_{j}^{(k)} \psi_{j}^{(k)}\right) d \sigma=0
$$

La dimostrazione del teorema (8.2) è evidente; quella del teorema (8.3) si ottiene facilmente sostituendo nel primo membro della (8.4) le $g_{j}^{(k)}$ con $\mathbf{i}$ primi membri corrispondenti delle (5.6), invertendo l'ordine di integrazione nell' integrale ripetuto e tenendo infine conto che, sussistendo le (8.3), sussistono anche le (8.1). Nostro scopo è di stabilire sotto quali ulteriori condizioni le (8.4) sono sufficienti per la risolubilità del problema I, ciò che otterremo facilmente tenendo presenti i risultati dal n. 5. Per altro il teorema (8.3) ci permetterà di riconoscere in maniera molto semplice che, in un esempio di problema II da noi riportato nel n. 9, vi sono infinite condizioni di compatibilità. Tenendo ora presente il teorema (8.1) ed i teoremi (5.1), (5.2) e $(5,3)$ si dimostra immediatamente il

Teorema $(8.4)$ - Se per la forma quadratica $(6.10)$ è verificata l'ipotesi $\mathrm{I}_{6}$, e per ogni $\xi \in A_{p} \cap B_{q}$ e qualunque sic $\tau \in \Pi_{\xi} \cap \partial \mathrm{\Omega}_{m}(\xi, 1)$ risulta:

$$
\operatorname{det} \mathscr{N}(p, q)(\xi, \xi, \tau) \neq 0,
$$


e ciò si verifica per ogni coppia di indici $p$ e q tali che $A_{p} \cap B_{g} \grave{e}$ non vuoto, si ha:

1) Il problema omogeneo asscciato al problema I ed il suo aggiunto ammettono un numero finito di soluzioni linearmente indipendenti, siano $k$ e $k^{*}$ rispettivamente.

2) Condizione necessaria e sufficiente perchè il problema I sia risolubile è che sia verificata la (84) per ogni soluzione $[v, \downarrow]$ del problema VI $e$ per questo occorre e basta che ne siano verificate $k^{*}$ in corrispondenza di altrettante soluzioni linearmente indipendenti del suddetto problema $\left({ }^{34}\right)$

3) Tutte e sole le soluzioni del problema, quando siano verificate le condizioni di compatibilità, sono fornite dalle formule (55) ponendo al posto di $\varphi$ la piu generale soluzione del sistema $\left(5.6^{\prime \prime}\right)$, che verrò a dipendere da th costanti arbitrarie.

Data l'equivalenza stabilita dal teorema (8.1) tra il problema I ed il sistema (5.6) la proposizione 1) è diretta conseguenza dei teoremi citati. Per quanto riguarda la proposizione 2), basta osservare che, quando sono verificate le condizioni di compatibilita, la o le soluzioni del sistema (5.6) esistono come elementi di $L^{(2)}\left(\{\Theta\}_{B}\right)$; per un recente (5) teorema di MIHLIN, essendo $g \in C^{(0, x)}\left(\left\{\Gamma_{A}\right\}\right)$, si deduce che ogni soluzione del sistema $(5.6)$ è di classe $C^{(0, \alpha)}\left(\{\Theta\}_{B}\right)$, e ciò assicnra che le funzioni (5.5) corrispondenti sono di classe $C^{(0, x)}(T) \cap C^{(1)}(T-\partial T)$ in virtù del teorema $(3.8)$.

Posto ora:

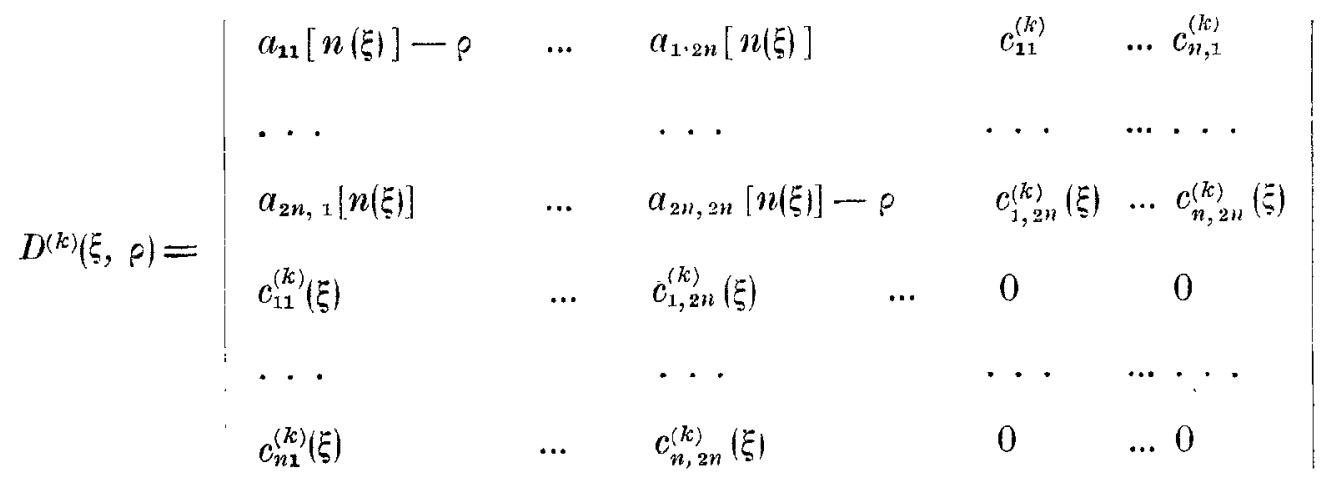

ci proponiamo di dimostrare il

(34) È evidente come debbano intendersi lineamente indipendenti un numero finito di soluzioni del problema VI.

$\left({ }^{35}\right)$ Cfr. quanto abbiamo detto a proposito della nota $\left({ }^{33}\right)$. 
Teorema $(8.5)$ - Se ciascuna delle equazioni $D^{(k)}(\xi, \rho)=0$ ammette per ogni fissato $\xi$ in $A_{k}$ e per ogni $k$, soltanto radici positive [negative], il problema I ammette al piì una soluzione.

Infatti, se $\left\{u_{1}^{0}, \ldots, u_{2}^{0}\right\}$ e una soluzione del problema omogeneo asso. ciato, si ricava facilmente, applicando il teorema di GaUsS

$$
\int_{\partial T}^{1, \ldots} \sum_{r, s}^{2 n} a_{r s}[n(\xi)] u_{r}^{0}(\xi) u_{s}^{0}(\xi) d \xi \sigma=0 .
$$

Faremo ora vedere che tale eguaglianza, se sono verificate le ipotesi del teorema, può sussistere se e soltanto se tutte le $u_{i}^{0}$ sono nulle identica. mente su $\partial T$. A tale scopo osserviamo che il minimo ed il massimo della forma quadratica

$$
\Phi(U)=\sum_{r, s}^{1, \ldots, 2 n} a_{r s}[n(\xi)] U_{r} U_{s}
$$

per ogni fissato $\xi \in A_{k}$, nell'insieme definito dalle condizioni

$(8.6)$

$$
\sum_{s=1}^{2 n} c_{r s}^{(k)}(\xi) U_{s}=0 \quad r=1, \ldots n
$$

$$
\sum_{s=1}^{2 n} U_{s}^{2}-1=0
$$

che indicheremo con $\{U\}_{i z, k}$, sono assunti in punti $U$ che devono soddisfare alle condizioni $(8.6)$ ed alle seguenti altre:

$$
\frac{\partial \Phi}{\partial U_{s}}+\sum_{r=1}^{n} \mu_{r} c_{r s}^{(k)}+2 p U_{s}=0, \quad s=1, \ldots, n
$$

avendo indicato con $\mu_{1}, \ldots, \mu_{n}, \rho$ i moltiplicatori indeterminati di LAGRANGE. Poichè si ricava facilmente che, per ogni soluzione

$$
\widetilde{U}_{1}, \ldots, \bar{U}_{2 n}, \bar{\mu}_{1}, \ldots, \bar{\mu}_{n}, \bar{\rho}
$$

del sistema di condizioni $(8.6)-(8.7)$ deve aversi

$$
\bar{\rho}=\Phi(\bar{U}),
$$

si deduce che tra i valori che competono a $p$ nelle soluzioni di tale sistema vi sono il minimo ed il massimo della $\Phi$. Poichè non possono essere tutte 
nulle le

$$
\bar{U}_{1}, \ldots, U_{2 n}, \bar{\mu}_{1}, \ldots, \mu_{n}
$$

in virtì della seconda delle $|8.6|$, si deduce facilmente che

$$
D^{(k)}(\xi, \bar{\rho})=0 \text {. }
$$

Si ha percio che, in virtù delle ipotesi poste, il massimo ed il minimo della $\Phi(U)$ sull'insieme $\{U\}_{5, k}$ sono entrambi positivi [negativi], qualunque sia $\xi \in A_{k}$ e qualunque sia $k$. Ne segue che la $\Phi(U)$ assume soltanto valori positivi in corrispondenza di valori non tutti nulli per le $U_{1}, \ldots, U_{2 n}$. Do. vendo sussistere la (8.5), si deduce che le funzioni $u_{i}^{0}$ sono identicamente uulle su $\partial T$. Tenendo presente che per le funzioni $u_{i}^{0}(x)$ devono valere le formule (5.2) con le $f_{r}$ identicamente nulle in $T$ si ricava che tutte le $u_{i}^{0}$ sono identicamente nulle in $T$, da cui segue facilmente il nostro asserto.

Ora osserviamo che, quando sono verificate le ipotesi del teorema (8.4), ogni ipotesi che implichi l'unicità per il problema VI implica pure che il problema I ammette soluzione qualunque siano i termini noti. Poichè il problema VI è dello stesso tipo del problema omogeneo associato al problema I (infatti l'eliminazione delle funzioni $\psi_{l}^{(k)}$ dalle (83) porta, per l'ipotesi $\mathrm{I}_{1}$ ), ad $n$ combinazioni lineari omogenee fra le $v_{s}^{+}$dello stesso tipo delle (1.3)) applicando il teorema di unicità (8.5), potremo avere delle condizioni sotto le quali per il problema I non vi sono condizioni di compatibilità. Senza addentrarci nlteriormente in questa questione, osserviamo che un teorema di unicità per il problema VI è il seguente

Trorema (8.6) - Se la forma quadratica

$$
\left.\Psi^{(k)}(\xi, \Delta)={\underset{j, l}{1, \ldots, n}}^{i, \ldots,{ }^{2 n}} A_{r s}[n(\xi)] c_{j r}^{(k)}(\xi) c_{l s}^{(k)}(\xi)\right) \Lambda_{j} \Lambda_{l}
$$

per quasi tutti $i$ punti $\xi \in A_{k}$, ̀̀ definita positiva [negativa] per ogni $k=1 . . ., N$, il problema VI non ammette soluzioni diversa da quella banale.

Il teorema si dimostra in base al fatto che, se $[v, \psi]$ è una soluzione del problema VI, dovendosi avere

$$
\int_{\partial T}^{1, \ldots{ }^{2}{ }^{2 n}} a_{r s}[n(\xi)] v_{r}^{+}(\xi) v_{s}+(\xi) d_{\xi} \sigma=0
$$


dalle $\left(5.1^{\prime}\right)$ e le $(8.3)$ si deduce

$$
\left.0=\sum_{k=1}^{N} \int_{D_{k}}^{1, \ldots, \ldots} \sum_{j, l}^{n}\left(\sum_{r, s}^{1, \ldots,{ }^{2 n}} A_{r s}[n(\xi)] c_{j r}^{(k)} \mid \xi\right) c_{l s}^{(k)}(\xi)\right) \psi_{j}^{(k)}(\xi) \psi_{l}^{(k)}(\xi) d \xi \sigma
$$

da cui segue $\psi \equiv 0$.

Possiamo ora enunciare il

Teorema (8.7) - Se sono verificate le ipotesi del teorema (8.4) e quella del teorema (8.6), il problema I ammette soluzioni, qualunque siano $i$ termini noti e l'omogeneo associato un mumero finito, eventualmente nullo. di soluzioni linearmente indipendenti; se sono verificate le ipotesi del teorema $(8.4)$ e quella del teorema (85), ammette al più una soluzione: ne ammette una ed una sola se sono verificate un numero finito di condizioni di compatibilitò.

Vogliamo rilevare che nel caso del problema III sono verificate le ipotesi dei teoremi (8.4), (8.5) e (8.6).

9) Alcuni esempi. - In questo numero fisseremo la nostra attenzione su una classe di sistemi del tipo (1.1) verificanti la seguente ipotesi

$\left.I_{7}\right)$ Per ogni $\lambda=\left(\lambda_{1}, \ldots, \lambda_{m}\right)$ si abbia :

$$
\sum_{r=1}^{2 n}\left\{\left(\sum_{p=1}^{m i} a_{t r}^{\nu} \lambda_{p}\right)\left(\sum_{q=1}^{m} a_{r s}^{q} \lambda_{q}\right)=\delta_{t s}\left(\lambda_{1}^{z}+\ldots+\lambda_{m}^{2}\right)\right.
$$

Si constata facilmente che un tale sistema è ellittico; supporremo in più che si abbia:

$$
\operatorname{det}\left\|\sum_{p=1}^{m} \alpha_{r s}^{p} \lambda_{p}\right\|=-\left(\lambda_{1}^{2}+\ldots+\lambda_{m}^{2}\right)^{n}
$$

Le (9.1) sono equivalenti alle seguenti condizioni

$$
\begin{array}{ll}
\sum_{r=1}^{2 n}\left(a_{t r}^{p} a_{r s}^{q}+a_{t r}^{q} a_{r s}^{p}\right)=0 & \text { per } t \neq s \\
\sum_{r=1}^{2 n} a_{s r}^{p} a_{r s}^{q}=\delta_{p q} . &
\end{array}
$$

Posto per comodità $\mathfrak{Z A}^{p}=\left\|a_{r s}^{p}\right\|$ per $p=1, \ldots, m$ lo (9.2) e le (9.2') si possono anche mettere sotto la seguente forma

$$
\begin{array}{ll}
\mathfrak{A}^{p} \cdot \mathfrak{A}^{q}+\mathfrak{A}^{q} \cdot \mathfrak{A}^{p}=0 & \text { pөr } p \neq q \\
\mathfrak{A}^{p} \cdot \mathfrak{A}^{p}=\mathbb{I} &
\end{array}
$$


avendo posto $\|=\| \delta_{r s} \|$. Si può far vedere che, ad esempio, per ogni intero positivo $m$ esistono $m$ matrici d'ordine $2^{m-1}$ che verificano le condizioni suddette: anzi $q u i$ ne determineremo $m$, ciascuna delle quali è simmetrica. Per dimostrare l'esistenza di queste $m$ matrici, ci serviremo di un procedimento ricorrente. Supponiamo che $\mathfrak{Z}_{m}^{(1)}, \ldots, \mathfrak{H}_{m}^{(m)}$ sono $\boldsymbol{m}$ ma. trici verificanti le condizioni (9.3) e non preoccupiamoci di determinare per ora l'ordine di queste matrici; consideriamo le $m+1$ matrici di ordine doppio a quello delle $\mathfrak{Z}_{(m)}^{p}$ definite nel modo seguente

$$
\begin{aligned}
& \mathfrak{A}_{(m+1)}^{p}=\left(\begin{array}{cc}
\mathfrak{H}_{(m)}^{p} & \mathbb{Q}_{(m)} \\
\mathbb{Q}_{(m)} & -\mathfrak{A}_{(m)}^{p}
\end{array}\right) \quad \text { per } p=1, \ldots, m, \\
& \mathfrak{A}_{(m+1)}^{m+1}=\left(\begin{array}{ll}
\mathbb{N}_{(m)} & \mathbb{I}_{(m)} \\
\mathbb{I}_{(m)} & \mathbb{Q}_{(m)}
\end{array}\right)
\end{aligned}
$$

avendo indicato con $\mathbb{Q}_{(m)}$ la matrice avente lo stesso ordine de $\mathfrak{A}_{(m)}^{p}$ avente tutti gli elementi nulli. 亡 facile vedere che le matrici $\mathfrak{Z}_{m+1}^{1}, \ldots, \mathfrak{Z}_{(m+1)}^{m+1}$ verificano ancora le condizioni (9.3).

In base a ciò, partendo dal caso $m=1$,

$$
\mathfrak{A}_{(1)}^{2}=\|-1\|
$$

si trovano, per ogni $m$ positivo, $m+1$ matrici verificanti le condizioni $(9.3)\left({ }^{36}\right)$.

Per un sistema che verifichi l'ipotesi $I_{7}$ si trova facilmente la matrice fondamentale in base alle seguenti considerazioni fondate su procedimenti di calcolo simbolico.

Posto

$$
\begin{aligned}
& \mathfrak{A}\left(\lambda_{1}, \ldots, \lambda_{m}\right)=\mathfrak{Z}^{1} \lambda_{1}+\ldots+\mathfrak{A}^{m} \lambda_{m} \\
& u=\left(u_{1}, \ldots, u_{2 n}\right)
\end{aligned}
$$

il sistema (1.1) si può mettere sotto la seguente forma matriciale:

$$
\mathfrak{Z}\left(\frac{\partial}{\partial x_{1}}, \ldots, \frac{\partial}{\partial x_{m}}\right) \cdot u=f
$$

(36) Rileviamo esplicitamente che per $m=2$ si trova così il sistema di equazioni dif. ferenziali che dà le condizioni di Cauchy-Riemann, pes $m=3$ si ottiene un sistema di equazioni differenziali equivalente a quello da noi studiato in un precodente lavoro cfr. Ric. di Mat. vol. I (1960). 
detta $\mathfrak{A}^{\prime p}$ la matrice trasposta di $\mathfrak{Z}^{p}$, si osserva facilmente che in base alle (9.3) si ha:

$$
\mathfrak{z}\left(\frac{\partial}{\partial x_{1}}, \ldots, \frac{\partial}{\partial x_{m}}, \cdot \mathfrak{z}^{\prime}\left(\frac{\partial g}{\partial x_{1}} \ldots, \frac{\partial g}{\partial x_{m}}\right)=\mathbb{I}\left(\Delta_{2} g\right)\right.
$$

per ogni $g$ di classe $C^{(2)}$. Si deduce di qui che una matrice fondamentale è

$$
\| M_{r s}\left(y-x \|=\frac{1}{\left(2-\frac{1}{2}\right) \omega_{n t}} \mathfrak{A}^{\prime}\left(\frac{\partial}{\partial x_{1}} \mid \frac{1}{|y-x|^{m-2}}, \ldots, \frac{\partial}{\partial x_{m}}\left\lceil y-\frac{1}{|y-x|^{m-2}} .\right.\right.\right.
$$

ciò̀

$$
M_{r s}(y-x)=\frac{1}{(2-1}-\sum_{\bar{p}) \omega_{m}}^{m} a_{s r}^{p} \frac{\partial}{\partial x_{p}} \frac{1}{|y-x|^{n-2}}
$$

avendo posto $\omega_{m}=\operatorname{mis} \partial \mathbf{S}_{m}$.

Proponiamoci ora di trattare il problema $I$ per un sistema a coefficiente costanti reali che verifichi l'ipotesi $I_{7}$ e sia simmetrico. Facciamo vedere prima di tutto che in questo casio si puó effettuare la costruzione delle matrici $\left\|d_{r s}^{(k)}\right\|$, che ei sono servite per tradurre il suddetto problema in un sistema di equazioni integrali equivalente; a tale scopo basterà dimostrare il

TEorema (9.1) - Se le costanti $a_{r s}^{p}$ verificano l'ipotesi $\left.I_{7}\right)$ e quella di simmetria, le radici dell' equazione

$$
\operatorname{det}\left\|\sum_{p=1}^{m_{i}} a_{r s}^{p} \lambda_{p} \quad \delta_{r s} \circ\right\|=0
$$

hanno, ciascuna, caratteristica costante rispetto $a \lambda$.

Infatti è chiaro che le radici della (9.5), per l'ipotesi di simmetria, sono tutte reali; d'altra parte per $\lambda \in \partial \Omega_{m}$ la matrice $\left\|\Sigma a_{r s}^{p} \lambda_{p}\right\|$ è ortogonale in virtù della ipotesi $\left.I_{7}\right)$; da cio segue, per un teorema di BRIosonI $\left({ }^{37}\right)$, che le radici dell'equazione (9.5) sono $1 \mathrm{e}-1$. In virtù del teorema (6.2) da noi dimostrato la (9.5) ha tante radici positive quante negative, e quindi 1 e -1 sono entrambe multiple di ordine $n$. Segue di qui facilmente il nostro asserto.

Ci proponiamo di calcolare le matrici simboliche del sistema di equazioni integrali, in cui si traduce il problema $I$, in questo caso. Si tratta di calco. lare le funzioni $\tilde{C}^{(r, s)}(\xi, \tau)$ mediante la formula $(5.8)$.

(37) Cfr. per esempio, [21]. 
Poichè non è difficile riconoscere che

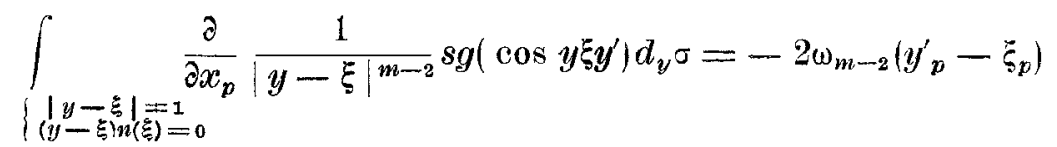

per ogni $y^{\prime}$ tale che $\left(y^{\prime}-\xi\right) \cdot n(\xi)=0$ si deduce facilmente che

$$
\tilde{O}^{(r, s)}(\xi, \tau)=\sum_{p=1}^{m} a_{r s}^{p} \tau_{p}
$$

essendo $\left(\tau_{1}, \ldots, \tau_{m}\right)$ un arbitrario vettore ortogonale ad $n(\xi)$.

Le matrici simboliche del sistema (5.6) in questo caso avranno la seguente espressione

$$
\mathscr{T K}^{\left(k, k^{\prime}\right)}(\xi, \xi, \tau)=\| \frac{1}{2}{ }_{r, s}^{1, \cdots,{ }^{2 n}}\left(\sum_{p=1}^{m} a_{r s}^{p}\left(n_{p}+i \tau_{p}\right) c_{j r}^{(k)}(\xi) d_{l s}^{\left(k^{\prime}\right)}(\xi) \|\right.
$$

per $\xi \in A_{k} \cap B_{k^{\prime}}$.

Vogliamo ora occuparci del problema III, cioè di quello che si ottiene sostituendo le $d_{j r}^{(k)}$ alle $c_{j r}^{(k)}$. In questo caso, tenendo presente come sono determinate le $d_{r s}^{(k)}$, per cui rinviamo al teorema (6.3), applicando le formule (6.4) si trova

$$
\underset{r, s}{1, \ldots,{ }^{2 n}} \sum_{p=1}^{m n} a_{r \mathrm{~s}}^{p} n_{p}(\xi) d_{j r}^{(k)}(\xi) d_{l s}^{(k)}(\xi) \equiv \delta_{j l} \quad \text { per } \xi \in B_{k} .
$$

Facciamo ora vedere che si ha

$$
\varphi_{j l}={ }_{r, s}^{1, \ldots, 2 n}\left(\sum_{p=1}^{m} \alpha_{r s}^{p} \tau_{p}(\xi)\right) d_{j r}^{(k)}(\xi) d_{l s}^{(k)}(\xi) \equiv 0
$$

Infatti dalla simmetria della matrice $\left\|{ }_{p=1}^{m} a_{r s}^{p} \tau_{p}(\xi)\right\|$, si ricava $\varphi_{\rho l}=\varphi_{l j}$; d'altro canto dalle condizioni (9.3) si deduce che $\varphi_{j l}=-\varphi_{l j}$, da cui seguono le (9.7). Per dimostrare che $\varphi_{i j}=-\varphi_{j l}$ osserviamo che, dalle formule (6.4) cui soddisfano le $d_{r s}^{(k)}$, si deduce

$$
d_{j r}^{(k)}=\sum_{h=1}^{2 n}\left(\sum_{q=1}^{m} a_{r h}^{q} n_{q}\right) d_{j h}^{(k)}
$$

da cui

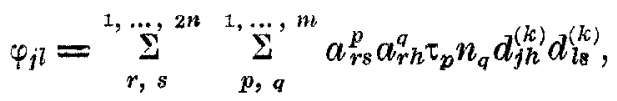


e, tenute presenti le (9.2) e che $n_{1} \tau_{1}+\ldots+n_{m} \tau_{m}=0$, si ha infine

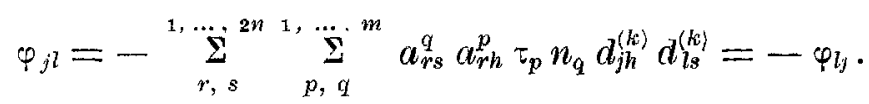

Si può concludere che le matrici simboliche in questo caso coincidono tutte con la matrice $\left\|\delta_{j l}\right\|$; da un'osservazione fatta nel n. 5), si deduce così il

Teorema (9.2). - Se $i$ coefficienti del sistema (1.1) verificano l' ipotesi $\mathrm{I}_{7}$ ), il problema III è equivalente ad un sistema di equazioni integrali del tipo di Frenholm di seconda specie.

Segnaliamo ora un caso in eui si hanno infinite condizioni di com. patibilità.

Cominciamo ad osservare che nella ipotesi $\left.I_{7}\right)$, se $\left\{u_{1}^{0}, \ldots, u_{2 n}^{0}\right\}$ è una soluzione del sistema omogeneo associato al sistema (1.1), ciascuna delle funzioni $u_{i}^{0}$ è armonica in $T-\partial T$; ciò si ricava dalla espressione della soluzione fondamentale, che permette di riconoscere attraverso la formula (5.2) che ciascuna $u_{i}^{0}$ è una combinazione lineare di derivate parziali prime di funzioni armoniche.

Proponiamoci ora di studiare il seguente

Problema VII - Determinare una soluzione del sistema (1.1) di classe $C^{(0,2)}(T) \cap C^{(1)}(T-\partial T)$ che su $\partial T$ verifichi le condizioni seguenti:

$$
\sum_{s=1}^{2 n}\left(\sum_{p=1}^{m} a_{r s}^{p} n_{p}\right) u_{s}=\beta_{r} . \quad \operatorname{per} r=1, \ldots, n,
$$

essendo $\beta_{1}, \ldots, \beta_{n} n$ assegnate funzioni di classe $C^{(0, \alpha)}(\partial T)$.

Trattando questo problema con lo stesso procedimento di cui ci siamo serviti nel n. 8) per trattare il problema I, troviamo come suo aggiunto il

Problema VIII. - Determinare le funzioni $\left\{v_{1}, \ldots, v_{2 n}\right\},\left\{\psi_{1}, \ldots, \psi_{n}\right\}$, le prime di classe $C^{(0, \alpha)}(T) \cap C^{(1)}(T-\partial T)$ e costituenti una soluzione del sistema onogeneo associato al sistema (1.1), le $\psi$ di classe $C^{(0, \alpha)}(\partial T)$ in modo che siano verificate le condizioni seguenti su $\partial T$ :

$$
\begin{array}{ll}
v_{s}=-\psi_{s} & \text { per } s=1, \ldots, n \\
v_{s}=0 & \text { per } s=n+1, \ldots, 2 n .
\end{array}
$$

Infatti le condizioni (8.3) in questo caso si riducono proprio alle (9.8) e alle $\left(9.8^{\prime}\right)$. Se ora supponiamo che le $a_{r s}^{p}$ verificano l'ipotesi $\left.I_{\gamma}\right)$, dalle $\left(9.8^{\prime}\right)$ ricaviamo che le $v_{n+1}, \ldots, v_{2 n}$ sono tutte nulle in $T$. Per avere una solu- 
zione $[\bar{v}, \bar{\psi}]$ del problema VII basta quindi considerare il sistema di equazioni formato dalle prime $n$ del sistema omogeneo associato al sistema (1.1), porre le $v_{n+1}, \ldots, v_{2 n}$ tutte uguali a zero, considerare il sistema che così si ottiene di $n$ equazioni (omogenee) nelle prime $n$ incognite, prendere una qualunque soluzione $v_{1}, \ldots, v_{n}$ di questo, e prendere infine le $\psi$ uguali alle tracce delle $\bar{v}_{1}, \ldots, \bar{v}_{n}$ cambiate di segno. Si comprende allora che, almeno per gli esempi effettivi da noi riportati di sistemi verificanti l'ipotesi $\mathrm{I}_{3}$, il problema VIII ammette infinite soluzioni linearmente indipendenti. In virtù del teorema (8.3), si può perciò concludere col

Teorema (9.3). - Se le matrici $\mathfrak{F}^{p} p=1, \ldots, m$ del sistema (1.1) sono quelle definite dalle velazioni ricorrenti (9.4), per il problema VII ci sono infinite condizioni di compatibilitì.

Osserviamo ora che, fissato un ricoprimento di $\mathfrak{z} T$, per formulare un problema con condizioni al contorno del tipo (1.3) e che non siano del tipo (1.5), occorre assegnare un sistema di coppie di matrici verificanti le condizioni (2.3). Mostriamo ora come un caso molto semplice (il più naturale, ci pare) di sistemi di matrici siffatte sia fornito dalla rappresentazione degli aperti che forniscono il ricoprimento di $\partial T$. Sía quindi, come in precedenza, $T$ di classe $A^{(2)}$ e $A_{1}, \ldots, A_{N}$ un ricoprimento finito di $\partial T$. Fissiamo su $A_{i}$ un sistema di coordinate curvilinee: $\left[t_{1}^{(i)}, \ldots, t_{m-1}^{(i)}\right]$ per $i=1, \ldots, N$. Essendo $T$ di classe $A^{(2)}$, per ogni insieme non vuoto del tipo $A_{i} \cap A_{j}$ esiste una trasformazione di classe $C^{(2)}$ con la sua inversa che permette di esprimere le coordinate di un punto qualunque appartenente ad $A_{i} \cap A_{l}$ nel sistema $\left[t_{1}^{(i)}, \ldots, t_{m-1}^{(i)}\right]$, mediante quelle dello stesso punto relative al sistema $\left[t_{1}^{(j)}, \ldots, t_{m-1}^{(j)}\right] ;$ tale trasformazione potremo indicare nel modo seguente:

$$
t_{k}^{(i)}=t_{k}^{(i)}\left(t_{1}^{\langle j}, \ldots, t_{m-1}^{(1)}\right) \quad k=1, \ldots, m-1
$$

Se ora si pone:

$$
\Gamma^{(i, j)}=\frac{\partial\left(t_{1}^{(i)}, \ldots, t_{m-1}^{(i)}\right)}{\partial\left(t_{1}^{(j)}, \ldots, t_{m-1}^{(j)}\right)}
$$

è facile verificare che $l^{\prime}$ insieme delle coppie di matrici $\Gamma^{(i, j)} \Gamma^{(3, i)}$ soddisfano alle condizioni (2.3), costituiscono cioè un sistema di matrici del tipo $\{\Gamma\}_{A, m-1}$. In questo caso lo spazio $C_{(0, x)}\left(\{\Gamma\}_{A, m-1}\right)$ è l'insieme dei vettori ad $m-1$ componenti localmente di classe $C^{(0, a)}$ su $\partial T$, che si tra sformano per controvarianza. In questo caso si ha anche un criterio per trasformare gli elementi di $C^{(0, \infty)}\left(\{\Gamma\}_{A, m-1}\right)$ quando ei si voglia riferire ad un ricoprimento di $\partial T$ diverso da $\{A\}_{N}$. Questo esempio mostra anche, indi- 
rettamente, che delle condizioni del tipo (1.3) non sono equivalenti, in generale, a condizioni del tipo (1.5), essendo ciò legato alla natura topologica di $\partial T^{(* *)}$

\section{BIBLIOGRAFIA}

[1] S. Agmon, A. Douglis and L. Niremberg, Boundary estimates for elliptic equations, "Comm. Pare Appl. Math. „, vol. XII n. 4, pp. 623-730.

[2] S. Banach, Théorie des Opérations Linéaires, «Hafner Publ. Company», Warsaw (1932).

[3] S. Bochner, Theta relations with spherical harmonics, \&Proc. of the Nat. Acad. of Sciences», vol. 37 (1951), pp. 804-808.

[4] S. Bochner and K. Chandrasekharan, Fourier Transforms, "Ann. of Mathemetics Studies *, $19(1949)$.

[5] A. P. Calderon and A. ZYGMund, On the existence of certain singular integrals, "Acta Math.», vel. 88 (1952), pp. 85-139.

[6] A. P. Calderon and A. Zygmund, On singular integrals, "Amer. J. Math.», vol. 78 (1956), pp. 289-309.

[7] A. P. CALderon and A. ZYGMUND, Algebras of certain singular operators, «Amer. J. Math. ", vol. 78 (1956), pp. 310.326.

[8] G. Frchera, Una introduzione alla teoria delle equazioni integrali singolari, «Rend di Mat. e delle sue Appl.", (1958).

[9] G. GIRAUd, Equations à intégrales principales, étude suivie d'une application. "Ann. Scient. Norm. Sup. s, t. 51 (1934), pp. 25̃1.372.

[10] G. GIRAUD, Sur une nouvelle catégorie d'équations oì figurent des valeurs principales d' intégrales, "Com. Rendus", t. 205 (1937), pp. 765.768.

[11] G. GIraud, Sur uno classe d'équations linéaires où figurent des valeurs principales d' intégrales simples, “Ann. Ec. Norm. sup.», t. 56 (1939), pp. 119.172.

[12] W. V. D. HoDGE, Theorie and applications of harmonic integrals, "Cambridge University Press.», $2^{a}$ ediz. (1952).

[13] F. JоHN, The fundamental solutions of linear elliptic differential equations with analitic coefficients, "Comm. on Pure and Applied Math.", 3 (1950), pp. 273-304.

[14] F. JоHN, Plane waves and spherical means applied to partial differential equations, Interscience publishers, ine. New York (195̄).

[15] S. G. Mrhuin, Singular Integral Equations, «Amer. Math. Soc. Translation», no 24 $(1950)$.

[16] S. G. MrhuIN, Composition of Multidimensional Singular Integral, Vest. Len. Univ. (1955)

$(* *)$ Nota aggiunta durante la correzione delle bozze. Attraverso la recenzione di un mio precedente lavoro [cfr. Ric. di Mat. vol. I (160)] apparsa sulla Mathematical Reviews, sono venuto a conoscenza di due memorie di R.T. SeELey: Singural integrals on compact manifold - Amer. Jour of Math. 1959; Regularization of singular integral operators on compact manifold, ibidem, 83. 1961. In queste memorie l'Autore oltre a stabilire nuovi interessanti risultati rielabora e completa quelli di MIHLiN e di GIRAUd relativi alla varietà. Riferendosi a questa rielaborazione sarebbe possibile esporre in una forma più generale e, per alcuni aspetti, più completa quei risultati che abbiamo richiamato nel n. 4 di questa memoria. 
[17] S. G. Mralin, Two theorems on regulavizers, Dokl Akad Nauk S SSR 125 (1959).

[18] S. G. Mrrur. Singular integral Equations in the classes of Lipschitz functions, Dokl. Akad. Nauk. S S S R 138:(1961).

[19] C. Miranda, Sull' integrazione delle forme differenziali esterne, Ricerche di Mat.», vol. II (1958).

[20] C. Mrranda, Equazioni alle derivate parziali di tipo ellitico, "Ergeb der Math. Sprin. ger-Verlag ", (1955).

[21] E. PAscal, I determinanti, Ulrico Hoepli, Milano (1928).

[22] F. TRIсом, Formula d'inversione dell"ordine di due integrazioni doppie "con asterisco" "Rend. Accad. Naz. Lincei», 6॰ (1926), pp. 535.539.

[23] F. Triconi, Equazioni integrali contenenti il valore principale di un integrale doppio, Math. Zeit. 2, 27 (1.928), pp. 87-133. 